\title{
Álgebras normadas de Dales-Davie
}

\author{
Vinícius Colferai Corrêa Miranda
}

\author{
DisSERTAÇ̃̃O APRESENTADA \\ AO \\ Instituto de Matemática e Estatística \\ DA \\ Universidade De SÃo Paulo \\ PARA \\ OBTENÇÃO DO TÍTULO \\ $\mathrm{DE}$ \\ Mestre em Matemática \\ Programa: Matemática \\ Orientador: Prof. Dra. Mary Lilian Lourenço
}

Durante o desenvolvimento deste trabalho o candidato recebeu apoio financeiro da FAPESP.

Processo número 2017/09301-4

São Paulo, 22 de Fevereiro de 2019 


\section{Álgebras normadas de Dales-Davie}

Esta é a versão original da dissertação elaborada pelo candidato Vinícius Colferai Corrêa Miranda, tal como submetida à Comissão Julgadora. 


\section{Álgebras normadas de Dales-Davie}

Esta versão da dissertação contém as correções e alterações sugeridas pela Comissão Julgadora durante a defesa da versão original do trabalho, realizada em 22/02/2019. Uma cópia da versão original está disponível no Instituto de Matemática e Estatística da Universidade de São Paulo.

Comissão Julgadora:

- Prof $^{\mathrm{a}}$. Dr ${ }^{\mathrm{a}}$. Mary Lilian Lourenço (orientadora) - IME-USP

- Prof. Dr. Walter Alberto de Siqueira Pedra - IF-USP

- Prof. Dr. Nilson da Costa Bernardes Junior - UFRJ 


\section{Agradecimentos}

Agradeço à minha mãe, Fátima, por sempre investir na minha educação, por todo o amor e por tudo o que fez por mim. Agradeço à minha família por todo o carinho e companhia ao longo desta jornada.

Gostaria de dedicar um parágrafo ao meu pai, Francelino, pelo amor e dedicação investido a mim enquanto pôde.

Agradeço à minha orientadora, Mary Lilian, por todo o aprendizado que eu tive desde a graduação. Por me aceitar como aluno de Iniciação Científica e de Mestrado. Por toda ajuda e oportunidades que me foram proporcionadas neste período.

Agradeço ao meu professor, Alberto, com quem tive aula no Ensino Médio e me despertou o desejo de cursar matemática no ensino superior. Agradeço aos professores que tive ao longo da graduação e do mestrado, com os quais aprendi muito.

Agradeço à minha companheira, Luana, por todo o tempo junto, pela paciência quando eu precisava estudar, pela ajuda nos momentos difíceis e diversão no tempo livre.

Quero agradecer aos meus amigos do instituto, por toda ajuda e por todas as risadas. Carlos, Marcos, Nathália, Reinaldo. Agradeço também aos meus amigos de fora do instituto. Em especial aos amigos do ME e do MacLaren's. Gostaria de agradecer às minhas companhias de futebol, em especial, ao Paulo e ao Guilherme por cada partida assistida no Brinco de Ouro da Princesa.

Agradeço à FAPESP pelo apoio financeiro durante o desenvolvimento do projeto - Processo 2017/09301-4. 


\section{Resumo}

MIRANDA, V. C. C. Álgebras normadas de Dales-Davie. FAPESP 2017/09301-4 Dissertação (Mestrado) - Instituto de Matemática e Estatística, Universidade de São Paulo, São Paulo, 2019.

Este trabalho tem como principal objetivo estudar as álgebras de Dales-Davie $D(X, M)$, onde $X$ é um subconjunto compacto e perfeito do plano complexo e $M=\left(M_{n}\right)$ é uma sequência de álgebra. Apresentamos aqui a construção dessas álgebras definidas por Dales e Davie em [11]. $\mathrm{Na}$ sequência, os principais resultados envolvendo a completude de tais álgebras encontrados nos textos $[7,11,19]$ e condições para que o seu espectro seja homeomorfo ao compacto $X$, assim como também apresentamos os espectros de duas subálgebras, $D_{R}(X, M)$ e $D_{P}(X, M)$, que foram estudadas nos textos $[2,11,18]$. Em seguida, estudamos os conceitos de lineabilidade, espaçabilidade e algebrabilidade com a finalidade de estudar os textos [25, 26] onde as autoras mostraram que o conjunto $\mathcal{A}(\mathcal{D}) \backslash D\left(\overline{\mathcal{D}},(n !)_{n \in \mathbb{N}_{0}}\right)$ é lineável, espaçável, algebrável e residual. 


\section{Abstract}

The main purpose of this work is to study the Dales-Davie algebras $D(X, M)$, where $X$ is a compact and perfect plane set and $M=\left(M_{n}\right)$ is an algebra sequence. We shall present the construction of this algebras defined by Dales and Davie in [11]. Following, the main results about its completeness as an normed algebra found in [7, 11, 19] and conditions that makes its spectrum be homeomorphic to $X$, besides that, we present the spectrum of two subalgebras, $D_{R}(X, M)$ and $D_{P}(X, M)$, such results cand be found in $[2,11,18]$. Furthermore, we study lineability, spaceability and algebrability in order to study the texts $[25,26]$ where the authors show that the set $\mathcal{A}(\mathcal{D}) \backslash D\left(\overline{\mathcal{D}},(n !)_{n \in \mathbb{N}_{0}}\right)$ is spaceable, algebrable and residual. 


\section{Sumário}

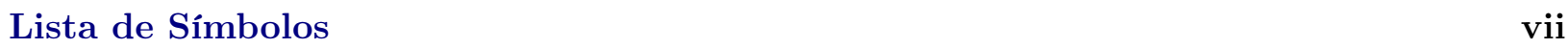

Introdução $\quad$ ix

1 Conceitos Preliminares 1

1.1 Tópicos em Análise Combinatória . . . . . . . . . . . . . . . . . . . . . . . . . . . . . 1

1.2 Noções de Topologia Geral . . . . . . . . . . . . . . . . . . . . . . . . . . . . . . . . . 2

1.3 Resultados de Análise Funcional . . . . . . . . . . . . . . . . . . . . . . . . . . . 7

1.4 Cardinalidade . . . . . . . . . . . . . . . . . . . . . . . . . 9

2 Álgebras de Funções $\quad 11$

2.1 Álgebras de Banach . . . . . . . . . . . . . . . . . . . . . . . . . . . . . . . 11

2.2 Álgebras Uniformes . . . . . . . . . . . . . . . . . . . . . . . . . . . . . . . 15

2.3 Álgebras Naturais . . . . . . . . . . . . . . . . . . . . . . . . . . . . 18

3 Álgebras de Dales-Davie $\quad 23$

3.1 Introdução . . . . . . . . . . . . . . . . . . . . . . . . . . . . . . . . . . . . 23

3.2 Completude em $D(X, M)$. . . . . . . . . . . . . . . . . . . . . . . . . . . . . . 26

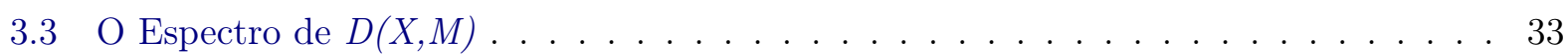

4 Lineabilidade e Algebrabilidade em um Subconjunto da Álgebra de Disco 45

4.1 Preliminares . . . . . . . . . . . . . . . . . . . . . . . 45

4.2 Lineabilidade e Espaçabilidade . . . . . . . . . . . . . . . . . . . . . . . . . . . . . . 47

4.3 Algebrabilidade . . . . . . . . . . . . . . . . . . . . . . . . . . 52

4.4 Residualidade . . . . . . . . . . . . . . . . . . . . . . . 56

Referências Bibliográficas $\quad 61$ 


\title{
Lista de Símbolos
}

\author{
$X \quad$ espaço topológico \\ $A \quad$ subconjunto de $X$ não vazio \\ $\bar{A} \quad$ fecho de $A$ \\ $A^{\circ} \quad$ interior de $A$ \\ $\partial A \quad$ fronteira de $A$ \\ card $(A)$ cardinalidade de $A$ \\ $\mathbb{N} \quad$ o conjunto dos números inteiros estritamente positivos \\ $\mathbb{N}_{0} \quad \mathbb{N} \cup\{0\}$ \\ $\mathbb{Z} \quad$ o conjunto dos números inteiros \\ $\mathbb{Q} \quad$ o corpo dos números racionais \\ $\mathbb{R} \quad$ o corpo dos números reais \\ $\mathbb{C} \quad$ o corpo dos números complexos \\ $\mathbb{K} \quad \mathbb{R}$ ou $\mathbb{C}$ \\ $\mathcal{D} \quad$ o disco unitário aberto em $\mathbb{C}$ \\ $\mathbb{C}[z] \quad$ a álgebra dos polinômios com coeficientes complexos \\ $K \quad$ espaço Hausdorff compacto \\ $C(K) \quad$ a álgebra das funções contínuas de $K$ em $\mathbb{C}$ \\ $\|f\|_{K} \quad \sup _{z \in K}|f(z)|$ \\ $R_{0}(K) \quad$ a álgebra das funções racionais com polos fora de $K$ \\ $R(K) \quad$ o fecho de $R_{0}(K)$ em $C(K)$
}




\section{Introdução}

\section{Considerações Preliminares}

O objetivo deste trabalho é estudar certas álgebras de funções infinitamente deriváveis. Se $X$ é um subconjunto compacto e perfeito de $\mathbb{C}$, dizemos que uma função $f: X \rightarrow \mathbb{C}$ é derivável em $z_{0} \in X$ se o seguinte limite existe:

$$
f^{\prime}\left(z_{0}\right)=\lim _{z \rightarrow z_{0}} \frac{f(z)-f\left(z_{0}\right)}{z-z_{0}}
$$

Em particular, uma função é derivável em $X$ se o é em todo ponto de $X$. A álgebra das funções $f: X \rightarrow \mathbb{C}$ com derivada contínua é denotada por $D^{1}(X)$. Vamos denotar por $D^{n}(X)$ a álgebra das funções com derivada de ordem $n$ contínua e por $f^{(n)}$ a $n$-ésima derivada de $f$. Enquanto $D^{\infty}(X)$ denotará a álgebra das funções infinitamente deriváveis em $X$.

Para cada $n \in \mathbb{N}$, temos que $\|f\|_{n}=\sum_{k=0}^{n} \frac{1}{k !}\left\|f^{(k)}\right\|_{X}$ define uma norma em $D^{n}(X)$. Uma questão natural é tentar estender esta norma para a álgebra $D^{\infty}(X)$. Não obstante, a série $\sum_{k=0}^{\infty} \frac{1}{k !}\left\|f^{(k)}\right\|_{X}$ não é necessariamente convergente. Desse modo, teríamos que estudar o seguinte espaço vetorial:

$$
\left\{f \in D^{\infty}(X): \sum_{k=0}^{\infty} \frac{1}{k !}\left\|f^{(k)}\right\|_{X}<\infty\right\} .
$$

Em 1973, Dales e Davie mostraram que o espaço acima é de fato uma álgebra normada de um modo mais geral. Mais precisamente, sejam $X \subset \mathbb{C}$ um conjunto compacto perfeito e $M=\left(M_{n}\right)$ uma sequência de números reais positivos que satisfaz:

$$
M_{0}=1 \text { e } \frac{M_{n}}{M_{k} M_{n-k}} \geq\left(\begin{array}{l}
n \\
k
\end{array}\right), k=1, \cdots, n, \forall n \in \mathbb{N} .
$$

Uma sequência definida dessa maneira é chamada de sequência de álgebra. Desse modo, definimos

$$
D(X, M)=\left\{f \in D^{\infty}(X): \sum_{n=0}^{\infty} \frac{1}{M_{n}}\left\|f^{(n)}\right\|_{X}<\infty\right\} .
$$

Em [11], Dales e Davie mostraram que $D(X, M)$ é uma álgebra normada com a norma:

$$
\|f\|=\sum_{n=0}^{\infty} \frac{1}{M_{n}}\left\|f^{(n)}\right\|_{X}, \forall f \in D(X, M) .
$$

O objetivo de Dales e Davie em [11] era encontrar condições sobre o conjunto $X$ tais que $D(X, M)$ 
seja uma álgebra de Banach.

Em 2007, tais álgebras foram denominadas álgebras de Dales-Davie por Abtahi e Honary [2]. Nesse tempo, diversos autores, e.g. Abtahi, Bland, Feinstein, Honary, Mahyar, estudaram a respeito dessas álgebras.

Em 1973, Dales e Davie apresentaram condições para que as álgebras $D(X, M)$ fossem completas. Quase 30 anos depois, Bland e Feinstein mostraram que $D(X, M)$ nem sempre é completa. Ainda em [11], Dales e Davie buscaram estudar o espectro de $D(X, M)$ e de duas subálgebras importantes $D_{R}(X, M)$ e $D_{P}(X, M)$ que são, respectivamente, os fechos de $R(X)$ e $\mathbb{C}[z]$ em $D(X, M)$. Dales e Davie mostraram que os espectros de $D_{R}(X, M)$ e de $D_{P}(X, M)$ são homeomorfos aos conjuntos $X$ e $\hat{X}$, respectivamente, onde $\hat{X}$ denota a envoltória polinomial convexa de $X$. O espectro de $D(X, M)$ continuou sendo estudado. Em 2007, Abtahi e Mahyar apresentaram condições para que o espectro de $D(X, M)$ seja homeomorfo ao conjunto $X$.

A ideia de estudar essas álgebras se deve ao fato de que ainda existem problemas em aberto nesta direção.

Se $X=\overline{\mathcal{D}}$, onde $\mathcal{D}$ é o disco unitário aberto do plano complexo, então $D(\overline{\mathcal{D}}, M)$ é uma subálgebra da álgebra de disco $\mathcal{A}(\mathcal{D})$. Uma pergunta natural é quão distante algébrica e topologicamente estão estas álgebras?

A resposta para esta pergunta está dentro do tema de algebrabilidade e residualidade sobre determinados conjuntos. Tal tópico aparece, por exemplo, em 1940 no texto [23] e em 1966 com Gurariy [16], mas sem a atual nomenclatura. A ideia era determinar espaços vetoriais de dimensão infinita em conjuntos de funções denominados patológicos. No inicio de século XXI, o interesse para estudos de estruturas de conjuntos patológicos voltam a aparecer com os trabalhos [4, 5, 17]. Assim, surgem as nomenclaturas atuais e o tópico passa a ser uma forte tendência com grande influência em análise real, análise complexa, teoria de operadores, polinômios em espaços de Banach, análise funcional em geral, entre outros. Para melhor informação sobre o tema, sugerimos os textos [6] e [3], o primeiro é um excelente survey e o segundo um livro que introduz o leitor na teoria e descreve as técnicas em diversas direções, bem como tem uma excelente bibliografia dos trabalhos publicados até 2016 .

Com este enfoque, Lourenço e Vieira estudaram a lineabilidade e algebrabilidade do conjunto $\mathcal{H}=\mathcal{A}(\mathcal{D}) \backslash D(\overline{\mathcal{D}}, M)[25,26]$, onde $M=\left(M_{n}\right)$ é uma sequência de álgebra que satisfaz $M_{n} \leq n !$, para todo $n \in \mathbb{N}$. Mais precisamente, Lourenço e Vieira mostraram que o conjunto é $\aleph_{0}$-lineável, c-lineável, espaçável, algebrável e fortemente c-algebrável.

Para estudar a residualidade, é necessário fazer uma pequena alteração no conjunto $\mathcal{H}$. Recordemos que

$$
f \in D(X, M) \Longleftrightarrow f \in D^{\infty}(X) \text { e } \sum_{k=0}^{\infty} \frac{1}{k !}\left\|f^{(k)}\right\|_{X}<\infty .
$$

Neste caso, vamos considerar

$$
\mathcal{H}=\left\{f \in D^{\infty}(X): \sum_{k=0}^{\infty} \frac{1}{k !}\left\|f^{(k)}\right\|_{X}=+\infty\right\} \subset \mathcal{A}(\mathcal{D}) .
$$

Desse modo, Lourenço e Vieira mostraram que o conjunto acima é residual em $\mathcal{A}(\mathcal{D})$. 


\section{Organização do Trabalho}

Nesta seção, descreverei os assuntos abordados em cada capítulo.

No capítulo 1, encontram-se algumas definições e resultados referentes à Análise Combinatória, Topologia Geral e Análise Funcional. Os resultados deste capítulo constam em [1, 8, 11, 13, 29].

No capítulo 2, intitulado Álgebras de Funções, apresentamos resultados que serão utilizados nos capítulos 3 e 4. Na primeira seção, definimos álgebras de Banach e apresentamos alguns resultados importantes, como, por exemplos a Fórmula do Raio Espectral e o Teorema de Gelfand. Na sequência definimos álgebras uniformes e álgebras naturais, como também apresentamos alguns exemplos e resultados nessa direção. Além disso, nessa seção são introduzidas algumas das principais álgebras trabalhadas nesse texto como $C(K), R(K)$ e $\mathcal{A}(\mathcal{D})$. O estudo feito neste capítulo pode ser encontrado no livro [15].

O capítulo 3 introduz as Álgebras de Dales-Davie, denotadas por $D(X, M)$. Na primeira seção, definimos $D(X, M)$ que é uma álgebra normada. Na sequência, estudamos a respeito da completude de $D(X, M)$. Mais precisamente, apresentamos as condições encontradas por Dales e Davie para que $D(X, M)$ seja uma álgebra Banach [11], como também estudamos a demonstração de que $D(X, M)$ nem sempre é completa [7]. Por fim, estudamos o espectro de $D(X, M)$ e de duas sub-álgebras de $D(X, M), D_{R}(X, M)$ e $D_{P}(X, M)$ que são, respectivamente, os fechos de $R(X)$ e $\mathbb{C}[z]$ em $D(X, M)$ $[2,11,18]$.

O capítulo 4 aborda os conceitos de lineabilidade, algebrabilidade e residualidade. Na primeira seção, apresentamos os pré-requisitos necessários para o desenvolvimento do capítulo que podem ser encontrados no livro [3]. Em seguida, estudamos os resultados obtidos por Lourenço e Vieira em $[25,26]$. 
xii INTRODUÇÃO 


\section{Capítulo 1}

\section{Conceitos Preliminares}

Neste capítulo, apresentamos os pré-requisitos para a leitura desta dissertação. Boa parte das demonstrações são estudas em disciplinas de nível de graduação e podem ser encontradas em [8, $13,24]$.

\subsection{Tópicos em Análise Combinatória}

Durante a dissertação, usaremos resultados de análise combinatória. No que segue, introduziremos algumas notações e fórmulas. Destacamos aqui a conhecida Fórmula de Faà di Bruno que permite calcular a n-ésima derivada de uma função composta. Faà di Bruno publicou sua fórmula em 1855 originalmente em italiano. Dois anos depois, o autor publicou uma versão em francês da demonstração de sua fórmula [14]. Uma outra demonstração também pode ser encontrada em [20].

Sejam $m, n \in \mathbb{N}_{0}$ com $n \geq m$. Considere o conjunto

$$
S(m, n)=\left\{\left(a_{1}, \cdots, a_{n}\right) \in \mathbb{N}_{0}^{n}: \sum_{k=1}^{n} a_{k}=m \text { e } \sum_{k=1}^{n} k a_{k}=n\right\} .
$$

Como comentamos acima, a Fórmula de Faà di Bruno permite calcular a n-ésima derivada de uma função composta. Se $\Omega \subset \mathbb{C}$ é um aberto e se $f: \Omega \rightarrow \mathbb{C}$ é uma função $n$-vezes derivável em $z_{0} \in \mathbb{C}$, vamos denotar a n-ésima derivada de $f$ em ponto $z_{0}$ por $f^{(n)}\left(z_{0}\right)$. Utilizando essas notações, podemos enunciar a Fórmula de Faà di Bruno.

Proposição 1.1.1. (Fórmula de Faà di Bruno) Seja $f$ uma função $n$-vezes derivável em $z \in \mathbb{C} e$ seja $F$ uma função $n$-vezes derivável em $f(z)$. Então, $F \circ f$ é $n$-vezes derivável em z e

$$
(F \circ f)^{(n)}(z)=\sum_{m=0}^{n} F^{(m)}(f(z)) \sum_{\left(a_{1}, \cdots, a_{n}\right) \in S(m, n)}\left[\frac{n !}{a_{1} ! \cdots a_{n} !} \prod_{k=1}^{n}\left(\frac{f^{(k)}(z)}{k !}\right)^{a_{k}}\right] .
$$

A Proposição 1.1.2 mostra uma variação da fórmula apresentada em 1.1.1 cuja demonstração pode ser encontrada em [20].

Proposição 1.1.2. (Fórmula de Hoppe) Sejam $f$ e $g$ duas funções com um número suficiente de derivadas, então

$$
(g \circ f)^{(n)}(z)=\sum_{r=1}^{n} \frac{g^{(r)}(f(z))}{r !} \sum_{s=0}^{r}\left(\begin{array}{l}
r \\
s
\end{array}\right)(-f(z))^{r-s}\left(f^{s}\right)^{(n)}(z) .
$$

A Proposição 1.1.3 pode ser encontrada na página 823 do livro [1]. Utilizando a mesma notação $S(m, n)$ definida anteriormente, temos: 
Proposição 1.1.3. Dados $m \in \mathbb{N}, t>0$ e $\left(x_{k}\right)$ sequência em $\mathbb{R}_{+}$, então

$$
\left(\sum_{k=1}^{\infty} \frac{x_{k}}{k !} t^{k}\right)^{m}=m ! \sum_{n=m}^{\infty} t^{n} \sum_{\left(a_{1}, \cdots, a_{n}\right) \in S(m, n)}\left(\prod_{k=1}^{n} \frac{x_{k}^{a_{k}}}{a_{k} ! k ! a_{k}}\right) .
$$

Como uma aplicação imediata da Proposição 1.1.3, temos o seguinte resultado que será utilizado no capítulo 3:

Corolário 1.1.4. Dados $m \in \mathbb{N} e\left(B_{m}\right)$ sequência de números reais positivos. Então,

$$
\left(\sum_{k=1}^{\infty} B_{k}\right)^{m}=m ! \sum_{n=m}^{\infty} \sum_{\left(a_{1}, \cdots, a_{n}\right) \in S(m, n)}\left(\prod_{k=1}^{n} \frac{B_{k}^{a_{k}}}{a_{k} !}\right) .
$$

Demonstração. É aplicação imediata da Proposição 1.1 .3 para $x_{k}=B_{k} k$ ! e $t=1$.

\subsection{Noções de Topologia Geral}

No que segue, vamos comentar alguns resultados e apresentar definições dentro do tópico de Topologia Geral que podem ser encontrados no livro [13]. Na sequência, definiremos o conjunto uniformemente regular que foi apresentado por Dales e Davie no texto [11]. Por fim, definiremos redes e apresentaremos alguns resultados nesta direção.

\section{Espaços Topológicos}

Para definirmos derivabilidade de funções no capítulo 2, precisaremos de conjuntos compactos e perfeitos. Conjuntos compactos são estudados em cursos elementares de Topologia Geral e, por isso, não serão abordados neste texto. Entretanto, definiremos conjuntos perfeitos que nem sempre são estudados em cursos básicos.

Definição 1.2.1. Sejam $X$ um espaço topológico e $A \subset X$. Dizemos que $A$ é um conjunto perfeito se for fechado e não possuir pontos isolados, i.e todo ponto de $A$ é um ponto de acumulação em $A$.

Exemplos 1.2.2. São exemplos de conjuntos perfeitos:

1. Se $X=\mathbb{R}$ e $A=[a, b]$ com $a<b$, então $A$ é perfeito.

2. $\operatorname{Em} X=\mathbb{C}$, bolas fechadas e esferas são conjuntos perfeitos.

Proposição 1.2.3. Seja $f: X \rightarrow Y$ uma função contínua entre os espaços topológicos $X$ e $Y$. Então:

1. Se $A \subset X$ compacto, $f(A)$ é compacto.

2. Se $A \subset X$ é perfeito e $f$ é injetora, $f(A)$ é perfeito.

Demonstração. O item 1 é um resultado bastante conhecido em topologia cuja demonstração pode ser encontrada na página 125 do livro [13]. Vejamos a demonstração do item 2:

Se $y \in f(A)$, existe $x \in A$ tal que $y=f(x)$. Para verificar que $f(A)$ é perfeito, é suficiente verificar que $y$ é um ponto de acumulação. Com efeito, seja $V$ uma vizinhança aberta de $y$ em $Y$. Pela continuidade da $f, f^{-1}(V)$ é uma vizinhança aberta de $x$ em $X$. Daí, como $x \in A$ e $A$ é perfeito, $x$ é um ponto de acumulação e, portanto, existe $x_{0} \in\left(f^{-1}(V) \backslash\{x\}\right) \cap A$. A injetividade de $f$ garante que $f\left(x_{0}\right) \neq y, \operatorname{logo} f\left(x_{0}\right) \in(V \backslash\{y\}) \cap f(A)$. Por conseguinte, temos que $f(A)$ é perfeito.

Proposição 1.2.4. Sejam $X$ um espaço compacto, $Y$ um espaço Hausdorff e $f: X \rightarrow Y$ uma função contínua e bijetora. Então, $f$ é um homeomorfismo. 
Demonstração. Sugerimos [13], pg. 125.

No capítulo 3, apresentaremos um resultado que utiliza componentes conexas. Por comodidade, definimos esta classe de conjuntos a seguir:

Definição 1.2.5. Sejam $X$ um espaço topológico e $x \in X$. A componente conexa de $x$ em $X$ é o maior conexo de $X$ que contém $x$.

Em particular, a componente conexa de $x \in X$ é

$$
C_{x}=\bigcup\{A \subset X: x \in A \text { e } A \text { conexo }\} .
$$

Observação 1.2.6. Seja $X$ um espaço topológico. Com a notação acima, temos:

1. Se $x, y \in X$ com $y \in C_{x}$, então $C_{x}=C_{y}$.

2. Componentes conexas são fechadas.

3. Se $X$ é conexo, então a única componente conexa de $X$ é o próprio $X$.

4. $X=\bigcup\left\{C_{x}: x \in X\right\}$.

Para um importante resultado do capítulo 4, precisamos estudar conjuntos residuais. A seguir, encontram-se as definições e os resultados necessários:

Definição 1.2.7. Sejam $X$ um espaço topológico e $A \subset X$.

1. $A$ é raro se $(\bar{A})^{\circ}=\emptyset$.

2. $A$ é residual se $X \backslash A$ é união enumerável de conjuntos raros.

Proposição 1.2.8. Sejam $X$ um espaço topológico e $A \subset X$. Então, A é raro se, e só se, $X \backslash A$ contém um aberto denso de $X$.

Demonstração. Usaremos aqui duas propriedades básicas sobre espaços topológicos, se $B \subset X$,

$$
(X \backslash B)^{\circ}=X \backslash \bar{B} \text { e } \overline{X \backslash B}=X \backslash B^{\circ} .
$$

Assim,

$$
\begin{aligned}
& (\bar{A})^{\circ}=\emptyset \Longleftrightarrow X \backslash(\bar{A})^{\circ}=X \\
& \Longleftrightarrow \overline{X \backslash \bar{A}}=X \\
& \Longleftrightarrow \overline{(X \backslash A)^{\circ}}=X \\
& \Longleftrightarrow(X \backslash A)^{\circ} \text { é denso em } X \text {. }
\end{aligned}
$$

Desse modo, se $A$ é raro, então $(X \backslash A)^{\circ}$ é um aberto denso de $X$ contido em $X \backslash A$. Reciprocamente, se $X \backslash A$ contém um aberto denso $B$ de $X$, então

$$
B \subset(X \backslash A)^{\circ} \Rightarrow(X \backslash A)^{\circ} \text { também é denso em } X \Rightarrow A \text { é raro. }
$$

Exemplos 1.2.9. Seja $X=\mathbb{R}$. São exemplos de conjuntos raros:

1. $\mathbb{Z}$ é raro em $\mathbb{R}$, pois $\mathbb{R} \backslash \mathbb{Z}$ é um aberto denso em $\mathbb{R}$.

2. $A=\left\{\frac{1}{n}: n \in \mathbb{N}\right\}$ é raro em $\mathbb{R}$, pois $\bar{A}=A \cup\{0\}$ que tem interior vazio. 
Observação 1.2.10. Sejam $X$ um espaço topológico e $A \subset X$. Se $A=\bigcap_{n=1}^{\infty} S_{n}$, onde cada $S_{n}$ é aberto denso de $X$, então A é residual.

De fato, segue da Proposição 1.2.8 uma vez que

$$
A=\bigcap_{n=1}^{\infty} S_{n} \Rightarrow X \backslash A=\bigcup_{n=1}^{\infty}\left(X \backslash S_{n}\right) \text { e } X \backslash\left(X \backslash S_{n}\right)=S_{n} \text { é aberto denso em } X .
$$

Apresentaremos um exemplo de conjunto residual, usando a Observação 1.2.10, no capítulo 4.

\section{Conjunto uniformemente regular}

Nesta subseção, apresentaremos a definição de conjunto uniformemente regular. Tais conjuntos foram introduzidos por Dales e Davie em [11] para apresentar situações em que as álgebras de Dales-Davie que serão definidas no capítulo 3 sejam completas. Antes disso, definiremos curvas no plano complexo:

Definição 1.2.11. Seja $X \subset \mathbb{C}$.

1. Uma curva em $X$ é uma função diferenciável por partes $\alpha:[0,1] \rightarrow X$.

2. O comprimento de arco de uma curva $\alpha$ em $X$ é definido por $L(\alpha)=\int_{0}^{1}\left|\alpha^{\prime}(t)\right| d t$.

3. Dizemos que $X$ é conexo por curvas retificáveis se dados $z_{1}, z_{2} \in X$, existe uma curva de comprimento finito $\alpha:[0,1] \rightarrow X$ que une $z_{1}$ e $z_{2}$, isto é $\alpha(0)=z_{1}$ e $\alpha(1)=z_{2}$. Além disso, neste caso definimos

$$
\delta\left(z_{1}, z_{2}\right)=\inf \left\{L(\alpha): \alpha \text { é curva em } X \text { que une } z_{1} \text { e } z_{2}\right\}<\infty .
$$

Note que o ínfimo acima existe, pois $\left\{L(\alpha): \alpha\right.$ é curva em $X$ que une $z_{1}$ e $\left.z_{2}\right\}$ é um conjunto limitado inferiormente.

Exemplos 1.2.12. São exemplos de conjuntos conexos por curvas retificáveis:

1. Bolas abertas e fechadas em $\mathbb{C}$.

2. A esfera unitária $S^{1}$ é conexo por curvas retificáveis, pois dados dois pontos $z, w \in S^{1}$, podemos considerar o arco unindo esses dois como uma curva em $S^{1}$ que terá comprimento finito. Neste exemplo, existirão pelo menos duas curvas que unem dois pontos, o maior e o menor arco. Logo, a métrica geodésica entre dois pontos em $S^{1}$ será o comprimento do menor arco, i.e o menor ângulo formado pelos pontos.

Dentro da área de geometria, temos o conhecido Teorema de Hopf-Hinow (e.g. [9]) que é enunciado para variedades Riemannianas conexas diz que um espaço métrico é completo se, e só se, é geodesicamente completo, i.e o ínfimo da métrica geodésica é atingido. Como $X \subset \mathbb{C}$ é compacto e conexo por curvas retificáveis, $X$ satisfaz as hipóteses do Teorema de Hopf-Hinow e é completo com a métrica euclidiana induzida. Desse modo, o ínfimo do conjunto

$$
\left\{L(\alpha): \alpha \text { é curva em } X \text { que une } z_{1} \text { e } z_{2}\right\}
$$

é atingido, isto é, dados $z_{1}$ e $z_{2}$ em $X$, existe uma curva $\alpha$ que une $z_{1}$ e $z_{2}$ com comprimento $\delta\left(z_{1}, z_{2}\right)$.

Lema 1.2.13. Seja $X \subset \mathbb{C}$ conexo por curvas retificáveis. A função $\delta: X \times X \rightarrow[0, \infty)$ definida em 1.2 é uma métrica em $X$, chamada de métrica geodésica em $X$. 
Demonstração. Para toda curva em $X$, temos $L(\alpha) \geq 0$. Assim, a imagem de $\delta$ está contida em $[0, \infty)$. Veremos agora que $\delta$ é de fato uma métrica. Com efeito, dado $z \in X$, a curva $\alpha(t)=z$, para todo $t \in[0,1]$, tem comprimento igual a 0 . Logo, $\delta(z, z)=0$. Por outro lado, dados $z, w \in X$ tais que $z \neq w$. Desse modo, toda curva $\alpha:[0,1] \rightarrow X$ que une $z$ e $w$ tem que ter comprimento não nulo. Assim, $\delta(z, w) \neq 0$.

Dados $z, w \in X$. Para toda curva $\alpha:[0,1] \rightarrow X$ tal que $\alpha(0)=z$ e $\alpha(1)=w$, considere uma curva $\gamma:[0,1] \rightarrow X$ dada por $\gamma(t)=\alpha(1-t)$. Daí, como $\gamma^{\prime}(t)=-\alpha^{\prime}(1-t)$, temos $L(\alpha)=L(\gamma)$. Então, $\delta(w, z) \leq \delta(z, w)$. A outra desigualdade segue de maneira análoga. Portanto, $\delta(z, w)=$ $\delta(w, z)$.

Dados $z_{1}, z_{2}, z_{3} \in X$. Sejam $\alpha$ curva unindo $z_{1}$ e $z_{2}$ e $\beta$ curva unindo $z_{2}$ e $z_{3}$. Considere $\gamma$ : $[0,1] \rightarrow X$ tal que $\gamma(t)=\alpha(2 t), 0 \leq t \leq 1 / 2$, e $\gamma(t)=\beta(2 t-1), 1 / 2<t \leq 1$. Daí, $\gamma$ está bem definida, é diferenciável e $\gamma(0)=z_{1}, \gamma(1)=z_{3}$ e $L(\alpha) \leq L(\beta)+L(\beta)$. Logo, $\delta\left(z_{1}, z_{3}\right) \leq$ $L(\alpha)+L(\beta)$. Isto implica em $\delta\left(z_{1}, z_{3}\right)-L(\beta) \leq L(\alpha)$, para toda curva $\alpha$ que une $z_{1}$ e $z_{2}$, portanto, $\delta\left(z_{1}, z_{3}\right)-L(\beta) \leq \delta\left(z_{1}, z_{2}\right) \Rightarrow \delta\left(z_{1}, z_{2}\right)-\delta\left(z_{1}, z_{2}\right) \leq L(\beta)$, para toda curva $\beta$ que une $z_{2}$ e $z_{3}$. Assim, concluímos que $\delta\left(z_{1}, z_{3}\right) \leq \delta\left(z_{1}, z_{2}\right)+\delta\left(z_{2}, z_{3}\right)$.

Em [11], Dales e Davie definiram dois conjuntos, o conjunto regular e o conjunto uniformemente regular. Os principais resultados encontrados na bibliografia, [2, 11], e que serão apresentados neste trabalho são para conjuntos uniformemente regulares. Desse modo, como não trabalharemos com o conjunto regular, omitiremos aqui sua definição que pode ser encontrada em [11].

Definição 1.2.14. Seja $X \subset \mathbb{C}$ um conjunto compacto, perfeito e conexo por curvas retificáveis. Dizemos que $X$ é uniformemente regular se existe $C>0$ tal que $\delta(z, w) \leq C|z-w|$, para todos $z, w \in X$.

Exemplos 1.2.15. 1. No disco unitário $\mathcal{D}$ e em $\overline{\mathcal{D}}$, a métrica geodésica coincide com a euclidiana, $\log o \mathcal{D}$ e $\overline{\mathcal{D}}$ são exemplos de conjuntos uniformemente regulares.

2. Sejam $z_{1}=e^{i \theta_{1}}$ e $z_{2}=e^{i \theta_{2}}$ pontos em $S^{1}$. Se $\theta_{1}-\theta_{2}=\delta+2 k \pi$, com $k \in \mathbb{Z}$ e $|\delta|<\pi$, temos que $\delta=\delta\left(z_{1}, z_{2}\right)$. Daí,

$$
\begin{aligned}
\left|e^{i \theta_{1}}-e^{i \theta_{2}}\right| & =\left|e^{i \theta_{2}}\right|\left|e^{i\left(\theta_{1}-\theta_{2}\right)}-1\right|=\left|e^{i\left(\theta_{1}-\theta_{2}\right)}-1\right|=\left|e^{i\left(\theta_{1}-\theta_{2}\right) / 2}\right|\left|e^{i\left(\theta_{1}-\theta_{2}\right) / 2}-e^{-i\left(\theta_{1}-\theta_{2}\right) / 2}\right| \\
& =\left|\left(\cos \frac{\theta_{1}-\theta_{2}}{2}+i \sin \frac{\theta_{1}-\theta_{2}}{2}\right)-\left(\cos \frac{\theta_{2}-\theta_{1}}{2}+i \sin \frac{\theta_{2}-\theta_{1}}{2}\right)\right| \\
& =\left|2 i \sin \frac{\theta_{1}-\theta_{2}}{2}\right|=2\left|\sin \frac{\theta_{1}-\theta_{2}}{2}\right|=2\left|\sin \frac{\delta}{2}\right|
\end{aligned}
$$

Vejamos que se $0 \leq \theta \leq \pi / 2$, então $\sin \theta \geq \frac{2}{\pi} \theta$. Com efeito, considere $f(x)=\sin x$ e $g(x)=\frac{2}{\pi} x$. Como $f$ é concava em $[0, \pi / 2]$, temos que $f(x) \geq g(x)$, para todo $x \in[0, \pi / 2]$. Daí,

$$
\left|e^{i \theta_{1}}-e^{i \theta_{2}}\right| \geq \frac{2}{\pi}|\delta|
$$

Desse modo, concluímos que $S^{1}$ é um conjunto uniformemente regular.

Apresentaremos, na sequência, dois lemas que serão utilizados em demonstrações de resultados no capítulo 3.

Lema 1.2.16. Sejam $X$ um conjunto uniformemente regular, $z_{1}, z_{2} \in X$ e $\alpha:[0,1] \rightarrow X$ curva que une $z_{1}$ e $z_{2}$ de comprimento $\delta\left(z_{1}, z_{2}\right)$. Então, $Y=\alpha([0,1])$ é uniformemente regular.

Demonstração. Uma vez que $\alpha$ é contínua e $[0,1]$ é compacto, segue do item 1 da item 1 da Proposição 1.2.3 que $Y$ é compacto. Por outro lado, pelo item 2 da Proposição 1.2.3, para garantir 
que $Y$ é perfeito, é suficiente verificar que $\alpha$ é injetora. Com efeito, se $\alpha$ não fosse injetora, existiriam $t_{1}<t_{2}$ em $[0,1]$ tais que $\alpha\left(t_{1}\right)=\alpha\left(t_{2}\right)$. Desse modo, a curva

$$
\gamma(t)= \begin{cases}\alpha\left(2 t_{1} t\right) & \text { se } 0 \leq t \leq 1 / 2 \\ \alpha\left(2\left(1-t_{2}\right) t+2 t_{2}-1\right) & \text { se } 1 / 2<t \leq 1\end{cases}
$$

une $z_{1}$ e $z_{2}$ e tem comprimento menor ou igual a $\alpha$. Neste caso, $\gamma$ tem comprimento $\delta\left(z_{1}, z_{2}\right)$ e pode substituir a $\alpha$. Como $[0,1]$ é compacto e $\alpha$ é contínua, podemos repetir o argumento um número finito vezes de modo a garantir que a curva seja injetora.

Dados $y_{1}, y_{2} \in Y$, existem $t_{1}, t_{2} \in[0,1]$ tais que $\alpha\left(t_{1}\right)=y_{1}$ e $\alpha\left(t_{2}\right)=y_{2}$. Sem perda de generalidade, suponha $t_{1}<t_{2}$. Daí, $\gamma=\left.\alpha\right|_{\left[t_{1}, t_{2}\right]}$ é uma curva que une $y_{1}$ e $y_{2}$ e tem comprimento finito. Logo, $Y$ é conexo por curvas retificáveis.

Finalmente, dados $z, w \in Y$, temos $\delta_{Y}(z, w)=\delta_{X}(z, w)$. De fato, como toda curva que une $z$ e $w$ é uma curva em $X$, sempre vale que $\delta_{Y}(z, w) \geq \delta_{X}(z, w)$. Se, por absurdo, $\delta_{Y}(z, w)>\delta_{X}(z, w)$, considere seja $\beta:\left[t_{1}, t_{2}\right] \rightarrow X$ curva de comprimento $\delta_{X}(z, w)$ que une $z$ e $w$. Daí, a função

$$
\gamma(t)= \begin{cases}\alpha(t) & \text { se } 0 \leq t \leq t_{1} \\ \beta(t) & \text { se } t_{1}<t<t_{2} \\ \alpha(t) & \text { se } t_{2} \leq t \leq 1\end{cases}
$$

é uma curva em $X$ unindo $z$ e $w$ com comprimento menor que $\delta(z, w)$, absurdo. Logo, $\delta_{Y}(z, w)=$ $\delta_{X}(z, w)$ e, por conseguinte, $\delta_{Y}(z, w) \leq C|z-w|$. Em suma, $Y$ é uniformemente regular.

Lema 1.2.17. Sejam $X, Y \subset \mathbb{C}$ dois conjuntos uniformemente regulares tais que $X \cap Y \neq \emptyset$. Então, $X \cup Y$ é uniformemente regular.

Demonstração. Como $X$ e $Y$ são conjuntos compactos e perfeitos, $X \cup Y$ também o é. Por hipótese, existe $z_{0} \in X \cap Y$. Então, dados $x \in X$ e $y \in Y$, podemos considerar as curvas de comprimento finito $\alpha:[0,1] \rightarrow X$ e $\beta:[0,1] \rightarrow Y$ que unem $x$ e $z_{0}$, e $z_{0}$ e $y$, respectivamente. Assim, a curva

$$
\gamma(t)= \begin{cases}\alpha(t) & \text { se } 0 \leq t \leq t / 2 \\ \beta(t) & \text { se } t / 2<t \leq 1\end{cases}
$$

tem comprimento finito. Além disso, se $C_{1}>0$ e $C_{2}>0$ são as constantes de regularidade uniforme em $X$ e $Y$, respectivamente, temos $\delta(x, y) \leq\left(C_{1}+C_{2}\right)|x-y|$, para todos $x, y \in X \cup Y$.

\section{Redes}

Utilizaremos em alguns resultados o conceito de Redes. Este tópico nem sempre é abordado em cursos elementares de Topologia Geral. Entretanto, é um conceito muito utilizado em diversos resultados de Análise Funcional.

No capítulo 2 do livro [29], o autor define redes em espaços topológicos e apresenta alguns resultados. Deixamos aqui este livro como sugestão ao leitor encontrar as demonstrações dos enunciados aqui apresentados.

Definição 1.2.18. Um conjunto não vazio $I$ munido de uma relação $\preceq$ é dito dirigido se satisfaz:

- $\alpha \preceq \alpha$, para todo $\alpha \in I$.

- Se $\alpha \preceq \beta$ e $\beta \preceq \gamma$, então $\alpha \preceq \gamma$.

- Dados $\alpha, \beta \in I$, existe $\gamma \in I$ tal que $\alpha \preceq \gamma$ e $\beta \preceq \gamma$.

Uma rede em um conjunto $X$ é uma função $f: I \rightarrow X$ definida num conjunto dirigido $I$. 
Se $f: I \rightarrow X$ é uma rede em $X$, escrevemos $f(\alpha)=x_{\alpha}$, para todo $\alpha \in I$. Além disso, denotamos $f=\left(x_{\alpha}\right)_{\alpha \in I}$. De modo análogo às sequências, vamos escrever $\left(x_{\alpha}\right)$.

Definição 1.2.19. Sejam $X$ um espaço topológico, $\left(x_{\alpha}\right)$ uma rede em $X$ e $x \in X$. Dizemos que $\left(x_{\alpha}\right)$ converge para $x$ se para cada vizinhança aberta $U$ de $x$ em $X$, existe $\alpha_{0} \in I$ tal que $x_{\alpha} \in U$, para todo $\alpha \in I$ com $\alpha_{0} \preceq \alpha$. Vamos denotar a convergência por $x_{\alpha} \rightarrow x$.

Em cursos elementares de análise, aprendemos a caracterizar espaços métricos através de sequências. Alguns desses resultados podem ser estendidos à espaços topológicos via redes. Enunciaremos aqui dois resultados que serão utilizados na dissertação.

Proposição 1.2.20. Sejam $X$ um espaço topológico, $S \subset X$ e $x \in X$. Então, $x \in \bar{S}$ se, e só se, existe uma rede $\left(x_{\alpha}\right)$ em $S$ tal que $x_{\alpha} \rightarrow x$.

Proposição 1.2.21. Sejam $X$ e $Y$ dois espaços topológicos. Uma função $f: X \rightarrow Y$ é contínua em $x \in X$ se, e só se, $f\left(x_{\alpha}\right) \rightarrow f(x)$ para toda rede $\left(x_{\alpha}\right)$ em $X$ que converge para $x$.

\subsection{Resultados de Análise Funcional}

Durante a dissertação, precisaremos de algumas definições e resultados de Análise Funcional. No que segue, apresentaremos resultados sobre Operadores Lineares Contínuos e Topologias Fracas. As definições e resultados desta seção podem ser encontrados em [8] e [29].

Nesta seção $E$ e $F$, salvo menção contrária, denotarão espaços normados. A bola unitária fechada de um espaço $E$ será denotada por $B_{E}$.

\section{Operadores lineares contínuos}

No capítulo 2 deste texto, introduziremos o conceito de homomorfismos de álgebras. Em particular, todo homomorfismo é um funcional linear contínuo. Desse modo, apresentaremos alguns resultados sobre operadores lineares contínuos.

Denotaremos por $\mathcal{L}_{a}(E, F)$ o espaço vetorial dos operadores lineares $T: E \rightarrow F$. Um operador $T \in \mathcal{L}_{a}(E, F)$ é contínuo se a função $T: E \rightarrow F$ é contínua considerando as topologias geradas pelas normas de $E$ e $F$. Um operador linear contínuo também é chamado de operador limitado. $O$ subespaço vetorial dos elementos contínuos de $\mathcal{L}_{a}(E, F)$ será denotado por $\mathcal{L}(E, F)$.

Além disso, um isomorfismo entre espaços de Banach é um operador linear contínuo invertível cuja inversa é um operador linear contínuo.

A Proposição 1.3.1 é um importante resultado sobre continuidade de operadores lineares.

Proposição 1.3.1. Se $T: E \rightarrow F$ é um operador linear, são equivalentes:

1. $T$ é contínuo.

2. T é contínuo na origem.

3. $\exists C>0,\|T(x)\| \leq C\|x\|, \forall x \in E$.

Além disso, a expressão

$$
\|T\|=\sup \left\{\|T(x)\|: x \in B_{E}\right\}
$$

define uma norma em $\mathcal{L}(E, F)$. Em particular, temos que

$$
T \in \mathcal{L}(E, F) \Longleftrightarrow\|T(x)\| \leq\|T\|\|x\|, \forall x \in E .
$$

Demonstração. Sugerimos [8], pg. 32 - 34 .

A seguir, enunciaremos o Teorema da Aplicação Aberta que é um importante resultado da área de Análise Funcional e que será utilizado na demonstração de um resultado no capítulo 3. 
Teorema 1.3.2. (Teorema da Aplicação Aberta) Sejam E e F espaços de Banach e $T: E \rightarrow F$ um operador linear, contínuo e sobrejetor. Então, $T$ é uma aplicação aberta. Em particular, todo operador linear contínuo e bijetor entre espaços de Banach é um isomorfismo.

Demonstração. Sugerimos [8], pg. 42.

Considere $E^{\prime}=\mathcal{L}(E, \mathbb{K})$ o espaço dual de $E$. Em particular, $E^{\prime}$ é sempre um espaço de Banach (Sugerimos [8], pg. 35), munido da norma

$$
\|\varphi\|=\sup \left\{|\varphi(x)|: x \in B_{E}\right\} .
$$

Desse modo, $E^{\prime \prime}=\left(E^{\prime}\right)^{\prime}$. Assim, podemos considerar o mergulho canônico $J_{E}: E \rightarrow E^{\prime \prime}, J_{E}(x)(\varphi)=$ $\varphi(x), x \in E, \varphi \in E^{\prime}$. Em particular, $J_{E}$ é uma isometria linear ([8], pg. 89).

\section{Topologias Fracas}

No próximo capítulo, estudaremos álgebras de Banach. Nesta direção, apresentaremos uma topologia no espectro de uma álgebra de Banach. Desse modo, precisamos estudar topologias fracas e fraca-estrelas.

Sejam $X$ um conjunto, $\left(Y_{i}\right)_{i \in I}$ uma família de espaços topológicos e $\mathcal{F}=\left(f_{i}\right)_{i \in I}$ uma família de funções $f_{i}: X \rightarrow Y_{i}$, para todo $i \in I$. Dados $i \in I$ e $A_{i}$ aberto em $Y_{i}$, defina

$$
f^{-1}\left(A_{i}\right)=\left\{x \in X: f_{i}(x) \in A_{i}\right\} .
$$

Denotemos por $\Phi$ a coleção dos subconjuntos de $X$ que podem ser escritos como interseções finitas de conjuntos da forma $f_{i}^{-1}\left(A_{i}\right)$

Proposição 1.3.3. Com a notação acima, existe uma topologia $\tau$ em $X$ que tem $\Phi$ como base. Além disso,

1. $\tau$ é a menor topologia em $X$ que torna todas as funções $f_{i}$ contínuas.

2. Seja $\left(x_{\alpha}\right)$ uma rede em $X$. Então, $x_{\alpha} \rightarrow x$ em $(X, \tau)$ se, e só se, $f_{i}\left(x_{\alpha}\right) \rightarrow f_{i}(x)$ em $Y_{i}$, para todo $i \in I$.

Demonstração. Sugerimos [29], pg. 203 - 204.

A topologia $\tau$ definida na Proposição anterior é chamada de topologia gerada pela família de funções $\left(f_{i}\right)_{i \in I}$ e denotada por $\sigma(X, \mathcal{F})$.

Em particular, o espaço $E^{\prime}$ é uma família de funções, motivando a seguinte definição:

Definição 1.3.4. A topologia fraca em $E$ é a topologia gerada por $E^{\prime}$, i.e $\sigma\left(E, E^{\prime}\right)$. Doravante, denotaremos a topologia fraca em $E$ por $(E, \omega)$.

Quando uma rede $\left(x_{\lambda}\right)$ em $E$ converge para $x \in E$ na topologia fraca, escrevemos $x_{\lambda} \stackrel{\omega}{\rightarrow} x$.

Proposição 1.3.5. Em um espaço normado E, temos:

1. A topologia fraca é Hausdorff.

2. Os funcionais lineares contínuos são fracamente contínuos.

3. Dados $x_{0} \in E, \varphi_{1}, \cdots, \varphi_{n} \in E^{\prime}$ e $\epsilon>0$, os conjuntos da forma

$$
U\left(x_{0}, \varphi_{1}, \cdots, \varphi_{n}, \epsilon\right)=\left\{x \in E: \sup _{1 \leq k \leq n}\left|\varphi_{k}(x)-\varphi_{k}\left(x_{0}\right)\right|<\epsilon\right\}
$$

formam uma base de vizinhanças abertas de $x_{0}$ na topologia fraca. 
4. Seja $\left(x_{\lambda}\right)$ uma rede em E. Então, $x_{\lambda} \rightarrow x \in E$ se, e somente se, $\varphi\left(x_{\lambda}\right) \rightarrow \varphi(x)$, para todo $\varphi \in E^{\prime}$.

Demonstração. Sugerimos [8], pg. 144.

Definição 1.3.6. A topologia fraca-estrela no dual $E^{\prime}$, denotada, por $\sigma\left(E^{\prime}, E\right)$ ou $\omega^{*}$, é a topologia em $E^{\prime}$ gerada pela família de funções $J_{E}(E)$. A topologia fraca-estrela em $E^{\prime}$ é denotada por $\left(E^{\prime}, \omega^{*}\right)$.

Quando uma rede $\left(\varphi_{\lambda}\right)$ em $E^{\prime}$ converge para $\varphi \in E^{\prime}$ na topologia fraca-estrela, escrevemos $\varphi_{\lambda} \stackrel{\omega^{*}}{\rightarrow} \varphi$

Proposição 1.3.7. Em um espaço normado E, temos:

1. A topologia fraca-estrela é Hausdorff.

2. Para todo $x \in E, J_{E}(x):\left(E^{\prime}, \omega^{*}\right) \rightarrow \mathbb{K}$ é continuo.

3. Dados $\varphi_{0} \in E^{\prime}, x_{1}, \cdots, x_{n} \in E$ e $\epsilon>0$, os conjuntos da forma

$$
V\left(\varphi_{0}, x_{1}, \cdots, x_{n}, \epsilon\right)=\left\{\varphi \in E^{\prime}: \sup _{1 \leq k \leq n}\left|\varphi\left(x_{k}\right)-\varphi_{0}\left(x_{k}\right)\right|\right\}
$$

formam uma base de vizinhanças abertas de $\varphi_{0}$ para a topologia fraca-estrela.

4. Seja $\left(\varphi_{\lambda}\right)$ uma rede em $E^{\prime}$. Então, $\varphi_{\lambda} \stackrel{\omega^{*}}{\rightarrow} \varphi \in E^{\prime}$ se, e só se, $\varphi_{\lambda}(x) \rightarrow \varphi(x), \forall x \in E$.

Demonstração. Sugerimos [8], pg. 152.

Teorema 1.3.8. (Teorema de Banach-Alaoglu-Bourbaki) Para todo espaço normado E, a bola $B_{E^{\prime}}$ é $\omega^{*}$ - compacta.

Demonstração. Sugerimos [8], pg. 156.

\subsection{Cardinalidade}

Para estudarmos lineabilidade e algebrabilidade que são conceitos que serão abordados no Capítulo 4 precisamos estudar cardinalidade de conjuntos. Em 2016, Aron, Bernal-González, Pellegrino e Seoane-Sepúlveda lançaram um livro reunindo os principais resultados dentro dos tópicos de lineabilidade e algebrabilidade. Para trabalhar nesses assuntos, os autores precisaram definir alguns conceitos de cardinalidade no capítulo 1 de [3].

Chamamos card $(A)$ de cardinalidade de $A$. Um cardinal card $(A)$ é dito:

1. finito se $A$ é finito; caso contrário é chamado de cardinal infinito.

2. enumerável se é finito ou card $(A)=\operatorname{card}(\mathbb{N})$.

Para conjuntos finitos, a cardinalidade de $A$ pode ser vista como a quantidade de elementos de $A$, i.e se existe uma bijeção entre $A$ e $\{1, \cdots, n\}$, temos card $(A)=n$.

Escreveremos também

$$
\operatorname{card}(\mathbb{N})=\aleph_{0} \text { e } \operatorname{card}(\mathbb{R})=\mathfrak{c} .
$$

É conveniente definir a cardinalidade do conjunto vazio como zero. Em particular, temos o seguinte resultado:

Proposição 1.4.1. Se $\alpha$ é um cardinal infinito, então $\aleph_{0} \leq \alpha$. 
Demonstração. Seja $M$ conjunto tal que card $(M)=\alpha$. Escolha $x_{1} \in M$. Como $M$ não é finito, existe $x_{2} \in M \backslash\left\{x_{1}\right\}$. Assim, indutivamente, conseguimos sequência $\left(x_{n}\right)_{n \in \mathbb{N}}$ de elementos dois a dois distintos em $M$. Desse modo, a função $i \in \mathbb{N} \mapsto x_{i} \in M$ é injetora.

Temos os seguintes resultados a respeito da cardinalidade em espaços vetoriais:

Teorema 1.4.2. Quaisquer duas bases em um espaço vetorial têm a mesma cardinalidade.

Demonstração. Sugerimos [3], pg. 13.

Teorema 1.4.3. Se $V$ é um espaço de Banach de dimensão infinita, então $\operatorname{dim} V \geq \mathfrak{c}$.

Demonstração. Sugerimos [3], pg. 13. 


\section{Capítulo 2}

\section{Álgebras de Funções}

Neste capítulo, vamos apresentar alguns resultados sobre álgebras de funções. Mais precisamente, começamos estudando o conceito de álgebras de Banach. Em seguida, apresentaremos alguns resultados sobre as álgebras uniformes e álgebras naturais. Os resultados deste capítulo podem ser encontrados em [15, 22].

\section{1 Álgebras de Banach}

Nesta seção, encontram-se definições e alguns resultados a respeito da teoria geral de álgebras de Banach que serão necessários para o desenvolvimento da dissertação.

Definição 2.1.1. Uma álgebra é um $\mathbb{K}$-espaço vetorial $\mathcal{A}$ munido de uma operação de multiplicação que satisfaz as seguintes propriedades:

- $a(b c)=(a b) c, \forall a, b, c \in \mathcal{A}$.

- $(a+b) c=a c+b c, \forall a, b, c \in \mathcal{A}$.

- $a(b+c)=a b+a c, \forall a, b, c \in \mathcal{A}$.

- $\lambda(a b)=(\lambda a) b=a(\lambda b), \forall a, b \in \mathcal{A}, \forall \lambda \in \mathbb{K}$.

Além disso, dizemos que $\mathcal{A}$ :

1. é comutativa se $a b=b a, \forall a, b \in \mathcal{A}$.

2. tem unidade se existe um elemento $1_{\mathcal{A}} \in \mathcal{A}$ tal que $1_{\mathcal{A}} a=a 1_{\mathcal{A}}=a, \forall a \in \mathcal{A}$.

3. é normada se existe uma norma $\|\cdot\|$ em $\mathcal{A}$ como espaço vetorial satisfazendo:

$$
\|a b\| \leq\|a\| \cdot\|b\|, \forall a, b \in \mathcal{A} .
$$

4. é uma álgebra de Banach se é uma álgebra normada e $\mathcal{A}$ é um espaço de Banach munido de tal norma.

Ademais, $\mathcal{B} \subset \mathcal{A}$ é uma subálgebra de $\mathcal{A}$ se $\mathcal{B}$ é um subespaço vetorial de $\mathcal{A}$ fechado por multiplicação, i.e para quaisquer $a, b \in \mathcal{B}$, temos $a b \in \mathcal{B}$.

A seguir, apresentaremos alguns exemplos de álgebras.

Exemplos 2.1.2. São exemplos de álgebras:

1. Seja $K$ um espaço Hausdorff compacto. $C(K)=$ conjunto de todas as funções $f: K \rightarrow \mathbb{C}$ contínuas é uma álgebra comutativa com unidade se considerarmos as operações usuais. Além disso, a aplicação $\|\cdot\|_{K}: C(K) \rightarrow[0, \infty)$ definida por $\|f\|_{K}=\sup _{z \in K}|f(z)|$ é uma norma em $C(K)$ que torna $C(K)$ uma álgebra de Banach. 
2. O conjunto dos polinômios com coeficientes complexos $\mathbb{C}[z]$ é uma álgebra comutativa com unidade. Em particular, se $K \subset \mathbb{C}$ é um compacto, $\mathbb{C}[z]$ é uma álgebra normada com a norma $\|\cdot\|_{K}$ definida acima. Entretanto, não é uma álgebra de Banach.

3. $R_{0}(K)=$ funções racionais com polos fora de $K$ é uma álgebra normada, quando munida da norma $\|\cdot\|_{K}$, onde $K \subset \mathbb{C}$ é um compacto. Não obstante, não é uma álgebra de Banach.

4. Seja $\Omega \subset \mathbb{C}$ um aberto não vazio. Vamos denotar por $\mathcal{H}(\Omega)$ a álgebra de todas as funções holomorfas $f: \Omega \rightarrow \mathbb{C}$. Se $\Omega$ é limitado, considere $\mathcal{A}(\Omega)=\mathcal{H}(\Omega) \cap C(\bar{\Omega})$ que é uma álgebra de Banach quando munido da norma do sup em $\Omega$.

Caso $\Omega=\mathcal{D}=\{z \in \mathbb{C}:|z|<1\}, \mathcal{A}(\mathcal{D})$ é chamada de álgebra de disco.

Aproveitando a definição de $\mathcal{A}(\Omega)$ acima, apresentaremos dois resultados importantes na teoria de funções analíticas que serão utilizados na dissertação.

Teorema 2.1.3. (Princípio do Módulo Máximo) Sejam $\Omega \subset \mathbb{C}$ um aberto limitado de $\mathbb{C}$ e $f \in \mathcal{A}(\Omega)$. Então, $\|f\|_{\bar{\Omega}}=\sup _{z \in \partial \Omega}|f(z)|$.

Demonstração. Sugerimos [10], pg. 128.

Teorema 2.1.4. Seja $f \in \mathcal{A}(\mathcal{D})$, então existe uma sequência de polinômios $\left(p_{n}\right)$ que converge uniformemente para $f$ em $\overline{\mathcal{D}}$, i.e $\left\|p_{n}-f\right\|_{\overline{\mathcal{D}}} \rightarrow 0$.

Demonstração. Sugerimos [30], pg. 16.

Neste trabalho, estamos interessados em álgebras que são comutativas e com unidade. Desse modo, no que segue desta seção, salvo menção contrária, $\mathcal{A}$ denotará uma álgebra comutativa com unidade. Em algumas situações, vamos precisar que $\mathcal{A}$ seja uma álgebra de Banach, neste caso, também vamos supor que $\mathcal{A}$ seja comutativa e tenha unidade. A seguir, apresentaremos as definições de elemento invertível numa álgebra, espectro de um elemento numa álgebra e do raio espectral.

Definição 2.1.5. Seja $a \in \mathcal{A}$.

1. Se $a \neq 0$, dizemos que $a$ é invertível em $\mathcal{A}$ se existe $b \in \mathcal{A}$ tal que $a b=1$. Neste caso, $b$ é chamado de inverso de $a$ e denotado por $a^{-1}$.

2. O espectro de $a$ em $\mathcal{A}$ é dado pelo conjunto

$$
\sigma(a)=\left\{\lambda \in \mathbb{C}: a-\lambda 1_{\mathcal{A}} \text { não é invertível }\right\} .
$$

3. O raio espectral de $a$ em $\mathcal{A}$ é definido por

$$
r(a)=\sup \{|\lambda|: \lambda \in \sigma(a)\} .
$$

Exemplo 2.1.6. Seja $K$ um espaço Hausdorff compacto. Então, para toda $f \in C(K)$, temos $\sigma(f)=f(K)$.

De fato, se $\lambda \in f(K), \exists x \in X, \lambda=f(x) \Rightarrow 0=f(x)-\lambda \Rightarrow$ a função $f-\lambda 1$ se anula em $K$, logo não é invertível. Reciprocamente, se $\lambda \in \sigma(f), f-\lambda 1$ não é invertível, i.e deve se anular em algum ponto de $K$. Daí, $\lambda=f(x)$ para algum $x \in K$.

Vamos mostrar no Teorema 2.1.8 que o conjunto dos elementos invertíveis em uma álgebra de Banach com unidade é um conjunto aberto e não vazio. Antes disso, precisamos do seguinte Lema:

Lema 2.1.7. Sejam $\mathcal{A}$ uma álgebra de Banach e $a \in \mathcal{A}$ tal que $\left\|1_{\mathcal{A}}-a\right\|<1$. Então, a é invertível. 
Demonstração. Seja $a \in \mathcal{A}$. Uma vez que $\left\|1_{\mathcal{A}}-a\right\|<1$, segue que a série geométrica $\sum_{k=1}^{\infty}\left\|1_{\mathcal{A}}-a\right\|^{k}$ é convergente. Mas como $\mathcal{A}$ é Banach, temos que $\sum_{k=1}^{\infty}\left(1_{\mathcal{A}}-a\right)^{k} \in \mathcal{A}$. Seja $b=1_{\mathcal{A}}+\sum_{k=1}^{\infty}\left(1_{\mathcal{A}}-a\right)^{k} \in$ $\mathcal{A}$. Daí, usando que $a=1_{\mathcal{A}}-\left(1_{\mathcal{A}}-a\right)$, obtemos:

$$
\begin{aligned}
a b & =a \cdot\left(1_{\mathcal{A}}+\sum_{k=1}^{\infty}\left(1_{\mathcal{A}}-a\right)^{k}\right) \\
& =a+\left(1_{\mathcal{A}}-\left(1_{\mathcal{A}}-a\right)\right) \cdot\left(\sum_{k=1}^{\infty}\left(1_{\mathcal{A}}-a\right)^{k}\right) \\
& =a+\sum_{k=1}^{\infty}\left(1_{\mathcal{A}}-a\right)^{k}-\sum_{k=1}^{\infty}\left(1_{\mathcal{A}}-a\right)^{k+1} \\
& =a+\left(1_{\mathcal{A}}-a\right)=1_{\mathcal{A}} .
\end{aligned}
$$

Logo, $a$ é invertível.

Teorema 2.1.8. Sejam $\mathcal{A}$ uma álgebra de Banach e $\mathcal{G}$ o conjunto dos elementos invertíveis de $\mathcal{A}$. Então, $\mathcal{G}$ é aberto não vazio.

Demonstração. Como $1_{\mathcal{A}} \in \mathcal{G}$, temos $\mathcal{G} \neq \emptyset$. Seja $a_{0} \in \mathcal{G}$ com inversa $b_{0}$. Vamos provar que $B\left(a_{0}, 1 /\left\|b_{0}\right\|\right) \subset \mathcal{G}$. Com efeito, seja $a \in \mathcal{A}$ tal que $\left\|a-a_{0}\right\|<1 /\left\|b_{0}\right\|$. Daí,

$$
\left\|b_{0} a-1_{\mathcal{A}}\right\|=\left\|b_{0} a-b_{0} a_{0}\right\| \leq\left\|b_{0}\right\|\left\|a-a_{0}\right\|<1 \Rightarrow b_{0} a \in \mathcal{G} .
$$

Então, existe $c_{1} \in \mathcal{A}$ tal que $1_{\mathcal{A}}=c_{1}\left(b_{0} a\right)=\left(c_{1} b_{0}\right) a$. Logo, $a \in \mathcal{G}$.

O Teorema 2.1.9 que será enunciado a seguir é um conhecido resultado no tópico de álgebras de Banach. Destacamos a Fórmula do Raio Espectral que será utilizada no capítulo 3.

Teorema 2.1.9. Sejam $\mathcal{A}$ uma álgebra de Banach e a $\in \mathcal{A}$. Então,

1. $\sigma(a)$ é um subconjunto não vazio de $\mathbb{C}$, compacto e está contido em $\{\lambda:|\lambda| \leq\|a\|\}$.

2. (Fórmula do Raio Espectral) Temos que

$$
r(a)=\lim _{n \rightarrow \infty}\left\|a^{n}\right\|^{1 / n} .
$$

Demonstração. Sugerimos [22], pg. 23.

Agora vamos definir homomorfismo de álgebras.

Definição 2.1.10. Um homomorfismo $\varphi: \mathcal{A} \rightarrow \mathbb{C}$ é um funcional linear multiplicativo, i.e $\varphi(a b)=\varphi(a) \varphi(b), \forall a, b \in \mathcal{A}$.

Exemplo 2.1.11. Sejam $K$ um espaço Hausdorff compacto e $\mathcal{A} \subset C(K)$ uma subálgebra. Para cada $x \in K$, defina $\varphi_{x}: \mathcal{A} \rightarrow \mathbb{C}$ por $\varphi_{x}(f)=f(x)$. Temos que $\varphi_{x}$ é um homomorfismo não nulo de $C(K)$ chamado de avaliação de $\mathcal{A}$ em $x$.

Proposição 2.1.12. Seja $\varphi$ um homomorfismo não nulo de um álgebra de Banach . Então,

1. $\varphi\left(1_{\mathcal{A}}\right)=1$.

2. Se b é invertivel, $\varphi\left(b^{-1}\right)=\varphi(b)^{-1}$.

3. $\varphi$ é contínuo e $\|\varphi\|=1$. 
Demonstração. Como $\varphi \neq 0$, existe $a \in \mathcal{A}, \varphi(a) \neq 0$.

1. Seja $a \in \mathcal{A}, \varphi(a) \neq 0$. Assim,

$$
\varphi(a)=\varphi\left(a 1_{\mathcal{A}}\right)=\varphi(a) \varphi\left(1_{\mathcal{A}}\right) \Rightarrow 1=\varphi\left(1_{\mathcal{A}}\right) .
$$

2. Se $b \in \mathcal{A}$ é invertível, temos

$$
1=\varphi\left(1_{\mathcal{A}}\right)=\varphi\left(b b^{-1}\right)=\varphi(b) \varphi\left(b^{-1}\right) \Rightarrow \varphi\left(b^{-1}\right)=\varphi(b)^{-1} .
$$

3. Seja $a \in \mathcal{A}, \varphi(a) \neq 0$. Pelo item 1 , temos que $1_{\mathcal{A}}-\frac{a}{\varphi(a)} \in \operatorname{ker} \varphi$. Do item 2 , segue que $1_{\mathcal{A}}-\frac{a}{\varphi(a)}$ não é invertível. Assim, pelo Lema 2.1.7,

$$
\|a / \varphi(a)\|=\left\|1_{\mathcal{A}}-\left(1_{\mathcal{A}}-a / \varphi(a)\right)\right\| \geq 1 \Rightarrow|\varphi(a)| \leq\|a\| \Rightarrow \varphi \text { é contínuo. }
$$

Além disso, como $\varphi\left(1_{\mathcal{A}}\right)=1$, temos $\|\varphi\|=1$.

Para enunciarmos o conhecido Teorema de Gelfand, precisamos estudar ideais em álgebras de Banach.

Definição 2.1.13. Um ideal $I$ em uma álgebra $\mathcal{A}$ é um subespaço vetorial de $\mathcal{A}$ satisfazendo:

$$
a \in \mathcal{A}, b \in I \Rightarrow a b \in I \text {. }
$$

Se $I \neq\{0\}$ e $I \neq \mathcal{A}$, dizemos que $I$ é um ideal próprio. Além disso, um ideal próprio é dito maximal se não existe ideal próprio $J$ tal que $I \subset J$ e $I \neq J$.

Exemplo 2.1.14. Em $C(K)$ para $K$ Hausdorff compacto, todos os ideias maximais são da forma

$$
J_{x}=\{f \in C(K): f(x)=0\}, x \in K .
$$

Proposição 2.1.15. Em uma álgebra de Banach $\mathcal{A}$, temos:

1. Se I é um ideal próprio de $\mathcal{A}, \bar{I}$ também o é.

2. Todo ideal maximal é fechado.

3. Todo ideal próprio está contido em um ideal maximal.

Demonstração. Note que o item 2 é consequência imediata de 1 . Com efeito, se $I$ é um ideal maximal, $\bar{I}$ é um ideal próprio tal que $I \subset \bar{I}$. Assim, $I=\bar{I}$. O item 3 é uma aplicação do Lema de Zorn e terá sua demonstração aqui omitida, sugerimos [27], pg. 122. Provemos, pois, o item 1.

Seja $I$ um ideal próprio em $\mathcal{A}$. Em particular, $\bar{I}$ é um subespaço vetorial de $\mathcal{A}$. Além disso, se $a \in \mathcal{A}$ e $b \in \bar{I}$, existe $\left(b_{\lambda}\right)$ rede em $I$ convergindo para $b$. Assim, $\left(a b_{\lambda}\right)$ é rede em $I$ convergindo para $a b$, logo $a b \in \bar{I}$. Pelo Teorema 2.1.8, o conjunto dos elementos invertíveis de $\mathcal{A}, \mathcal{G}$, é um aberto não vazio. Então, $\mathcal{A} \backslash \mathcal{G}$ é um fechado e $I \subset \mathcal{A} \backslash \mathcal{G}$. Portanto, $\bar{I} \subset \mathcal{A} \backslash \mathcal{G} \neq \mathcal{A}$.

Sejam $\mathcal{A}$ uma álgebra e $J$ um ideal próprio fechado em $\mathcal{A}$. Definimos o quociente de $\mathcal{A}$ por $J$ pelo conjunto

$$
\mathcal{A} / J=\{a+J: a \in \mathcal{A}\} \text {, onde } a+J=\{a+b: b \in J\}, \forall a \in \mathcal{A} .
$$

Considerando as operações canônicas de soma, multiplicação e multiplicação por escalar, temos que $\mathcal{A} / J$ é uma álgebra. Além disso, se $\|\cdot\|_{\mathcal{A}}$ é uma norma para $\mathcal{A},\|a+J\|=\inf \left\{\|a+x\|_{\mathcal{A}}: x \in J\right\}$ define uma norma em $\mathcal{A} / J$. 
Teorema 2.1.16. Sejam $\mathcal{A}$ uma álgebra de Banach comutativa e com unidade e $J$ um ideal próprio fechado em $\mathcal{A}$. Então, o quociente $\mathcal{A} / J$ é uma álgebra de Banach comutativa e com unidade. Ademais, a projeção canônica $\pi: \mathcal{A} \rightarrow \mathcal{A} / J$ é um homomorfismo sobrejetor.

Demonstração. Sugerimos [27], pg. 122.

Em uma álgebra de Banach $\mathcal{A}$, vamos denotar por $\mathcal{M}_{\mathcal{A}}$ o conjunto de todos os homomorfismos não nulos em $\mathcal{A}$. Este conjunto é chamado de espectro de $\mathcal{A}$. A seguir, estudaremos dois importantes resultados sobre $\mathcal{M}_{\mathcal{A}}$.

Teorema 2.1.17. Em uma álgebra de Banach $\mathcal{A}$, para todo $\varphi \in \mathcal{M}_{\mathcal{A}}$, $\operatorname{ker} \varphi$ é um ideal maximal em $\mathcal{A}$. Reciprocamente, dado um ideal maximal I em $\mathcal{A}$, existe $\varphi \in \mathcal{M}_{\mathcal{A}}$ tal que $\operatorname{ker} \varphi=I$.

Demonstração. Sugerimos [27], pg. 124.

Teorema 2.1.18. Seja $\mathcal{A}$ uma álgebra de Banach. Então, $\mathcal{M}_{\mathcal{A}}$ é $\omega^{*}$-Hausdorff compacto.

Demonstração. $\mathcal{M}_{\mathcal{A}}$ é $\omega^{*}$-Hausdorff, pois é subespaço topológico do espaço $\left(\mathcal{A}^{\prime}, \omega^{*}\right)$ que é Hausdorff (Proposição 1.3.7 - 1). Como todo homomorfismo não nulo é contínuo e tem norma 1 (Proposição 2.1 .12 - 3), temos $\mathcal{M}_{\mathcal{A}} \subset B_{\mathcal{A}^{\prime}}$. Por outro lado, $B_{\mathcal{A}^{\prime}}$ é $\omega^{*}$-compacto (Teorema de Banach-AlaogluBourbaki - 1.3.8), assim é suficiente provar que $\mathcal{M}_{\mathcal{A}}$ é fechado.

Com efeito, seja $\left(\varphi_{\lambda}\right)$ uma rede em $\mathcal{M}_{\mathcal{A}}$ convergindo para $\varphi$ na topologia fraca-estrela. Dados $a, b \in \mathcal{A}$ e $\alpha \in \mathbb{K}$, temos

$$
\begin{gathered}
\varphi_{\lambda}(a b) \rightarrow \varphi(a b) \text { e } \varphi_{\lambda}(a b)=\varphi_{\lambda}(a) \varphi_{\lambda}(b) \rightarrow \varphi(a) \varphi(b) \Rightarrow \varphi(a b)=\varphi(a) \varphi(b) . \\
\varphi_{\lambda}(\alpha a+b) \rightarrow \varphi(\alpha a+b) \text { e } \varphi_{\lambda}(\alpha a+b)=\alpha \varphi_{\lambda}(a)+\varphi_{\lambda}(b) \rightarrow \alpha \varphi(a)+\varphi(b) \Rightarrow \varphi(\alpha a+b)=\alpha \varphi(a)+\varphi(b) .
\end{gathered}
$$

Daí, $\varphi$ é um homomorfismo. Mas como, $1=\varphi_{\lambda}\left(1_{\mathcal{A}}\right) \rightarrow \varphi\left(1_{\mathcal{A}}\right)$, temos $\varphi\left(1_{\mathcal{A}}\right)=1$. Portanto, $\varphi \in \mathcal{M}_{\mathcal{A}}$.

Para cada $a \in \mathcal{A}$, defina $\hat{a}: \mathcal{M}_{\mathcal{A}} \rightarrow \mathbb{C}$ da seguinte maneira:

$$
\hat{a}(\varphi)=\varphi(a), \forall \varphi \in \mathcal{M}_{\mathcal{A}} .
$$

Em particular, $\hat{a}$ é uma função contínua em $\mathcal{M}_{\mathcal{A}}$. Dizemos que $\hat{a}$ é a transformada de Gelfand de $a$. Desse modo, definimos a transformação de Gelfand de $\mathcal{A}$ pela aplicação $\Gamma: \mathcal{A} \rightarrow C\left(\mathcal{M}_{\mathcal{A}}\right)$ tal que $\Gamma(a)=\hat{a}$. A seguir, enunciaremos, sem demonstrar, o conhecido Teorema de Gelfand.

Teorema 2.1.19. (Teorema de Gelfand) Seja $\mathcal{A}$ uma álgebra de Banach. A transformação de Gelfand de $\mathcal{A}$ é um homomorfismo continuo entre álgebras de Banach. Além disso,

$$
\|\hat{a}\|_{\mathcal{M}_{\mathcal{A}}}=r(a) \leq\|a\| \quad \text { e }\|\Gamma\|=1 .
$$

Demonstração. Sugerimos [27], pg. 133.

\section{2 Álgebras Uniformes}

Nessa seção, introduziremos os conceitos de álgebras uniformes e fecho uniforme de uma álgebra de funções. Também apresentaremos exemplos importantes e resultados que serão utilizados no próximo capítulo. Para um estudo mais detalhado sobre o tema, sugerimos o livro [15].

Doravante, salvo menção contrária, o conjunto $K$ denotará um espaço Hausdorff compacto. Dizemos que um subconjunto não vazio $\mathcal{A}$ de $C(K)$ separa pontos se dado $x \in K$, existem $f, g \in \mathcal{A}$ tais que $f(x) \neq g(x)$.

Note que é imediato da definição que se um conjunto separa pontos, ele não pode ser unitário.

Definição 2.2.1. Seja $\mathcal{A}$ uma subálgebra de $C(K)$. Dizemos que $\mathcal{A}$ é uma álgebra de funções em $K$ se $\mathcal{A}$ é uma subálgebra de $C(K)$ que separa pontos e contém as constantes. Além disso, 
1. se existir uma norma $\|\cdot\|_{\mathcal{A}}$ para a álgebra $\mathcal{A}$, dizemos que $\mathcal{A}$ é uma álgebra normada de funções em $K$. Ademais, se $\mathcal{A}$, munida de tal norma, for Banach, dizemos que $\mathcal{A}$ é uma álgebra de Banach de funções em $K$.

2. Uma álgebra uniforme em $K$ é uma álgebra de funções em $K$ que é fechada com a norma do sup em $K$.

Exemplos 2.2.2. Seja $K \subset \mathbb{C}$ um espaço compacto. Temos que:

1. $R_{0}(K)$ é uma álgebra de funções em $K$ que não é uniforme.

2. $\mathcal{A}(\mathcal{D})$ é uma álgebra uniforme em $\mathcal{D}$.

A seguir, apresentaremos uma classe de exemplos de funções deriváveis que motivou a definição das álgebras de Dales e Davie que serão apresentadas no capítulo 3.

Exemplo 2.2.3. Seja $X \subset \mathbb{C}$ um conjunto compacto e perfeito, dizemos que uma função $f: X \rightarrow \mathbb{C}$ é derivável em $z_{0} \in \mathbb{C}$ se existe o seguinte limite:

$$
f^{\prime}\left(z_{0}\right)=\lim _{z \rightarrow z_{0}} \frac{f(z)-f\left(z_{0}\right)}{z-z_{0}} .
$$

Neste caso, $f^{\prime}\left(z_{0}\right)$ é a derivada de $f$ em $z_{0}$.

Dizemos que uma função $f: X \rightarrow \mathbb{C}$ é derivável em $X$ se $f$ é derivável em todo ponto de $X$. Em particular, toda função derivável é contínua. Denotaremos por $D^{1}(X)$ a álgebra das funções deriváveis em $X$ com derivada contínua em $X$.

A álgebra das funções com $n$-ésima derivada contínua em $X$ é denotada por $D^{n}(X)$, enquanto que a álgebra das funções com derivadas de todas as ordens será denotada por $D^{\infty}(X)$. Para cada $k \in \mathbb{N}$, denotaremos a $k$-ésima derivada de $f$ por $f^{(k)}$.

Além disso,

$$
\|f\|_{n}=\sum_{k=0}^{n} \frac{1}{k !}\left\|f^{(k)}\right\|_{X}, \forall f \in D^{n}(X),
$$

define uma norma em $D^{n}(X)$.

Proposição 2.2.4. Seja $\mathcal{A}$ uma álgebra de funções em $K$. O fecho uniforme de $\mathcal{A}$, i.e

$$
\overline{\mathcal{A}}=\left\{f \in C(K): \exists\left(f_{n}\right) \text { sequência em } \mathcal{A} \text { tal que }\left\|f_{n}-f\right\|_{K} \rightarrow 0\right\},
$$

é uma álgebra uniforme em $K$.

Demonstração. Note que $\mathcal{A} \subset \overline{\mathcal{A}}$. Logo, $\overline{\mathcal{A}} \neq \emptyset$ e $0 \in \overline{\mathcal{A}}$.

Sejam $f, g \in \overline{\mathcal{A}}$ e $\lambda \in \mathbb{C}$. Então, existem $\left(f_{n}\right)$ e $\left(g_{n}\right)$ sequências em $\mathcal{A}$ tais que $\left\|f_{n}-f\right\|_{K} \rightarrow 0$ e $\left\|g_{n}-g\right\|_{K} \rightarrow 0$. Vamos mostrar primeiro que $\lambda f+g \in \overline{\mathcal{A}}$. Com efeito, dado $\epsilon>0$, existem $n_{1}, n_{2} \in \mathbb{N}$ tais que

$$
\left\|f_{n}-f\right\|_{K}<\frac{1}{2|\lambda|} \epsilon, \forall n \geq n_{1}, \quad \text { e } \quad\left\|g_{n}-g\right\|_{K}<\frac{1}{2} \epsilon, \forall n \geq n_{2} .
$$

Assim, para todo $n \geq \max \left\{n_{1}, n_{2}\right\}$, temos

$$
\left\|\left(\lambda f_{n}+g_{n}\right)-(\lambda f+g)\right\|_{K} \leq|\lambda|\left\|f_{n}-f\right\|_{K}+\left\|g_{n}-g\right\|_{K}<\epsilon \Rightarrow\left\|\left(\lambda f_{n}+g_{n}\right)-(\lambda f+g)\right\|_{K} \rightarrow 0 .
$$

Veremos agora que $f g \in \overline{\mathcal{A}}$. Se $g=0$, temos $f g=0 \in \overline{\mathcal{A}}$. Desse modo, suponha que $g \neq 0$. Uma vez que a sequência $\left(f_{n}\right)$ é convergente, existe $M \geq 0$ tal que $\left\|f_{n}\right\| \leq M, \forall n \in \mathbb{N}$. Desse modo, dado $\epsilon>0$, existem $n_{1}, n_{2} \in \mathbb{N}$ tais que

$$
\left\|f_{n}-f\right\|_{K}<\frac{1}{2\|g\|_{K}} \epsilon, \forall n \geq n_{1}, \quad \text { e } \quad\left\|g_{n}-g\right\|_{K}<\frac{1}{2 M} \epsilon, \forall n \geq n_{2} .
$$


Daí, para todo $n \geq \max \left\{n_{1}, n_{2}\right\}$,

$$
\left\|f_{n} g_{n}-f g\right\|_{K} \leq\left\|f_{n}\right\|\left\|g_{n}-g\right\|+\left\|f_{n}-f\right\|_{K}\|g\|_{K}<\epsilon \Rightarrow\left\|f_{n} g_{n}-f g\right\|_{K} \rightarrow 0 .
$$

Portanto, $f g, \lambda f+g \in \overline{\mathcal{A}}$. Logo, $\overline{\mathcal{A}}$ é subálgebra de $C(K)$. Além disso, como $\overline{\mathcal{A}}$ é fechado em $C(K)$ com a norma induzida, segue que $\overline{\mathcal{A}}$ é álgebra uniforme em $K$.

Exemplos 2.2.5. Seja $K \subset \mathbb{C}$ compacto. Então,

1. $P(K)=\left\{f \in C(K): \exists\left(p_{n}\right) \subset \mathbb{C}[z]\right.$ tal que $\left.\left\|p_{n}-f\right\|_{K} \rightarrow 0\right\}$ é uma álgebra uniforme em $K$.

2. $R(K)=\left\{f \in C(K): \exists\left(r_{n}\right) \subset R_{0}(K)\right.$ tal que $\left.\left\|r_{n}-f\right\|_{K} \rightarrow 0\right\}$ é uma álgebra uniforme em $K$.

Proposição 2.2.6. Seja $\left(\mathcal{A},\|\cdot\|_{\mathcal{A}}\right)$ uma álgebra de Banach de funções em $K$. Então, $\forall f \in \mathcal{A}$,

$$
\|f\|_{K} \leq\|\hat{f}\|_{\mathcal{M}_{\mathcal{A}}} \leq\|f\|_{A},
$$

onde $\hat{f}$ é a transformada de Gelfand de $f$.

Demonstração.

$$
\|f\|_{K}=\sup _{x \in K}|f(x)|=\sup _{x \in K}\left|\varphi_{x}(f)\right| \leq \sup _{\varphi \in \mathcal{M}_{\mathcal{A}}}|\varphi(f)|=\|\hat{f}\|_{\mathcal{M}_{\mathcal{A}}} \leq\|f\|_{\mathcal{A}} .
$$

O próximo resultado apresenta uma condição necessária e suficiente para que $\mathcal{M}_{\overline{\mathcal{A}}}$ e $\mathcal{M}_{\mathcal{A}}$ sejam homeomorfos.

Teorema 2.2.7. Seja $\mathcal{A}$ uma álgebra de Banach de funções em $K$. Então, a restrição $F: \mathcal{M}_{\overline{\mathcal{A}}} \rightarrow$ $\mathcal{M}_{\mathcal{A}}, F(\varphi)=\left.\varphi\right|_{\mathcal{A}}$, é um homeomorfismo se, e só se, $\|\hat{f}\|_{\mathcal{M}_{\mathcal{A}}}=\|f\|_{K}, \forall f \in \mathcal{A}$.

Demonstração. Em particular, a restrição $F: \mathcal{M}_{\overline{\mathcal{A}}} \rightarrow \mathcal{M}_{\mathcal{A}}$ é sempre injetora e contínua. De fato, seja $\psi_{\lambda} \stackrel{\omega^{*}}{\rightarrow} \psi$ em $\mathcal{M}_{\overline{\mathcal{A}}}$. Assim, $\psi_{\lambda}(f) \rightarrow \psi(f)$ em $\mathbb{C}$ para toda função $f \in \overline{\mathcal{A}}$. Em particular, $\forall f \in \mathcal{A}, \psi_{\lambda}(f) \rightarrow \psi(f)$. Logo, $F\left(\psi_{\lambda}\right) \stackrel{\omega^{*}}{\rightarrow} F(\psi) \Rightarrow F$ é contínua.

Para verificarmos a injetividade, sejam $\psi, \varphi \in \mathcal{M}_{\overline{\mathcal{A}}}$ tais que $F(\psi)=F(\varphi)$. Se $f \in \overline{\mathcal{A}}$, existe sequência $\left(f_{n}\right)$ em $\mathcal{A}$ tal que $\left\|f_{n}-f\right\|_{K} \rightarrow 0$. Daí,

$$
|\varphi(f)-\psi(f)| \leq\left|\varphi(f)-\varphi\left(f_{n}\right)\right|+\left|\psi\left(f_{n}\right)-\psi(f)\right| \leq 2\left\|f-f_{n}\right\|_{K} \rightarrow 0 \Rightarrow \varphi=\psi .
$$

Suponha agora que $\|\hat{f}\|_{\mathcal{M}_{\mathcal{A}}}=\|f\|_{K}, \forall f \in \mathcal{A}$, vejamos que $F$ é sobrejetora. Com efeito, dados $\varphi \in \mathcal{M}_{\mathcal{A}}$ e $f \in \overline{\mathcal{A}}$, existe sequência $\left(f_{n}\right)$ em $\mathcal{A}$ tal que $\left\|f_{n}-f\right\|_{K} \rightarrow 0$. Em particular, $\left(f_{n}\right)$ é sequência de Cauchy em $C(K)$, assim $\forall \epsilon>0, \exists N_{0} \in \mathbb{N}, \forall n, m \geq N_{0},\left\|f_{n}-f_{m}\right\|_{K}<\epsilon$. Daí,

$$
\left|\varphi\left(f_{n}\right)-\varphi\left(f_{m}\right)\right| \leq\left\|\hat{f_{n}}-\hat{f_{m}}\right\|_{\mathcal{M}_{\mathcal{A}}}=\left\|f_{n}-f_{m}\right\|_{K}<\epsilon, \forall n, m \geq N_{0} .
$$

Portanto, $\left(\varphi\left(f_{n}\right)\right)$ é sequência de Cauchy em $\mathbb{C}$, logo existe $\lambda=\lim \varphi\left(f_{n}\right) \in \mathbb{C}$. Se $\left(g_{n}\right)$ é uma outra sequência em $\mathcal{A}$ tal que $\left\|g_{n}-f\right\|_{K} \rightarrow 0$, temos $\mu=\lim \varphi\left(g_{n}\right)$. Provemos que $\lambda=\mu$.

$$
\begin{aligned}
|\lambda-\mu| & \leq\left|\lambda-\varphi\left(f_{n}\right)\right|+\left|\varphi\left(f_{n}\right)-\varphi\left(g_{n}\right)\right|+\left|\varphi\left(g_{n}\right)-\mu\right| \\
& \leq\left|\lambda-\varphi\left(f_{n}\right)\right|+\left\|\hat{f}_{n}-\hat{g_{n}}\right\|_{\mathcal{M}_{\mathcal{A}}}+\left|\varphi\left(g_{n}\right)-\mu\right| \\
& \leq\left|\lambda-\varphi\left(f_{n}\right)\right|+\left\|f_{n}-g_{n}\right\|_{K}+\left|\varphi\left(g_{n}\right)-\mu\right| \\
& \leq\left|\lambda-\varphi\left(f_{n}\right)\right|+\left\|f_{n}-f\right\|_{K}+\left\|f-g_{n}\right\|_{K}+\left|\varphi\left(g_{n}\right)-\mu\right|
\end{aligned}
$$


Como cada um dos termos do lado direito converge para zero quando $n \rightarrow \infty$, temos $\lambda=\mu$. Assim, $\psi(f)=\lim \varphi\left(f_{n}\right), \forall f \in \overline{\mathcal{A}}$ e $\left(f_{n}\right)$ sequência em $\mathcal{A}$ convergindo uniformemente para $f$ em $K$, está bem definida. Em particular, $\psi \in \mathcal{M}_{\overline{\mathcal{A}}}$ e $F(\psi)=\varphi$.

Reciprocamente, suponha que $F$ seja um homeomorfismo. Assim, pela sobrejetividade de $F$, $\forall \varphi \in \mathcal{M}_{\mathcal{A}}, \exists \psi \in \mathcal{M}_{\overline{\mathcal{A}}}, F(\psi)=\varphi$. Daí,

$$
|\varphi(f)|=|\psi(f)| \leq\|f\|_{K}, \forall f \in \mathcal{A} \Rightarrow\|\hat{f}\|_{\mathcal{M}_{\mathcal{A}}}=\sup _{\varphi \in \mathcal{M}_{\mathcal{A}}}|\varphi(f)| \leq\|f\|_{K}, \forall f \in \mathcal{A} .
$$

A outra desigualdade decorre da Proposição 2.2.6.

No capítulo 3, vamos mostrar que o conjunto $D^{1}(X)$ definido no exemplo 2.2 .3 está contido em $R(X)$. Para isso, precisaremos de um teorema de [15].

Para apresentarmos tal resultado, necessitamos dos seguintes operadores:

$$
\frac{\partial}{\partial z}=\frac{1}{2}\left(\frac{\partial}{\partial x}-i \frac{\partial}{\partial y}\right) \quad \text { e } \quad \frac{\partial}{\partial \bar{z}}=\frac{1}{2}\left(\frac{\partial}{\partial x}+i \frac{\partial}{\partial y}\right) .
$$

Além disso, segue das equações de Cauchy-Riemann que $g$ é holomorfa num aberto $\Omega \subset \mathbb{C}$ se, e só se, $\frac{\partial g}{\partial \bar{z}}=0$. Neste caso, $\frac{\partial g}{\partial z}=g^{\prime}$.

Teorema 2.2.8. Sejam $X \subset \mathbb{C}$ compacto e $f \in C(X)$. Se existem $\Omega \subset \mathbb{C}$ aberto contendo $X$ e $F: \Omega \rightarrow \mathbb{C}$ função com derivada contínua tal que $\left.F\right|_{X}=\left.f e \frac{\partial F}{\partial \bar{z}}\right|_{X}=\frac{\partial f}{\partial \bar{z}}=0$. Então, $f \in R(X)$.

Demonstração. Sugerimos [15], pg. 26.

\section{3 Álgebras Naturais}

Nesta seção, vamos introduzir o conceito de álgebras naturais. Algumas técnicas que serão apresentadas aqui serão utilizadas no próximo capítulo.

Seja $J: K \rightarrow \mathcal{M}_{\mathcal{A}}$ a aplicação definida por $J(x)=\varphi_{x}$, isto é $J(x)(f)=\varphi_{x}(f)=f(x)$, para todos $x \in K$ e $f \in \mathcal{A}$. Vamos mostrar que $J$ é uma aplicação injetora e contínua. De fato, se $J(x)=J(y)$, então $f(x)=f(y), \forall f \in \mathcal{A}$. Como $\mathcal{A}$ separa pontos de $K$ segue que $x=y$. Por conseguinte, $J$ é injetora. Seja $\left(x_{\lambda}\right)$ rede em $K$ convergindo para $x \in K$. Por continuidade, $f\left(x_{\lambda}\right) \rightarrow f(x) \in \mathbb{C}, \forall f \in \mathcal{A}$. Logo, $\varphi_{x_{\lambda}}(f) \rightarrow \varphi_{x}(f), \forall f \in \mathcal{A}$, daí $\varphi_{x_{\lambda}} \stackrel{\omega^{*}}{\rightarrow} \varphi_{x}$, i.e $J\left(x_{\lambda}\right) \stackrel{\omega^{*}}{\rightarrow} J(x)$. Portanto, $J$ é contínua.

Definição 2.3.1. Seja $\mathcal{A}$ uma álgebra de Banach de funções em $K$. Dizemos que $\mathcal{A}$ é natural se a aplicação $J$ definida acima é um homeomorfismo, i.e $K$ e $\mathcal{M}_{\mathcal{A}}$ são homeomorfos.

Tendo em vista a Proposição 1.2.4, para que $\mathcal{A}$ seja uma álgebra natural, é suficiente verificar que $J$ é sobrejetora. A seguir, vamos mostrar que $C(K)$ e $\mathcal{A}(D)$ são álgebras naturais. Mas, antes disso, precisamos do seguinte Lema:

Lema 2.3.2. Sejam $\varphi$ e $\psi$ dois homomorfismos não nulos numa álgebra de Banach $\mathcal{A}$. Então, se $\operatorname{ker} \varphi=\operatorname{ker} \psi, \operatorname{temos} \varphi=\psi$.

Demonstração. Suponha $\operatorname{ker} \varphi=\operatorname{ker} \psi$. Então, para todo $a \in \mathcal{A}, a-1 \cdot \varphi(a) \in \operatorname{ker} \varphi=\operatorname{ker} \psi$. Daí,

$$
0=\psi(a-1 \cdot \varphi(a))=\psi(a)-\varphi(a) \Rightarrow \psi(a)=\varphi(a), \forall a \in \mathcal{A} .
$$

Exemplo 2.3.3. $C(K)$ é uma álgebra natural. 
De fato, suponha, por absurdo, que existe $\varphi \in \mathcal{M}_{C(K)}$ tal que $\varphi \neq \varphi_{x}, \forall x \in K$. Do Lema 2.3.2, temos $\operatorname{ker} \varphi \neq \operatorname{ker} \varphi_{x}, \forall x \in K$. Como núcleos são maximais, não pode haver uma inclusão própria. Desse modo,

$$
\forall x \in K, \exists f_{x} \in \operatorname{ker} \varphi, \varphi_{x}\left(f_{x}\right) \neq 0 \text {, i.e } f_{x}(x) \neq 0 .
$$

Por continuidade, existe uma vizinhança $V_{x}$ de $x$ em $X$ tal que $f_{x}(y) \neq 0, \forall y \in V_{x}$. Em particular, $\left\{V_{x}: x \in K\right\}$ é cobertura aberta para o compacto $X$, então existem $x_{1}, \cdots, x_{n} \in X$ tais que $K=\bigcup_{i=1}^{n} V_{x_{i}}$. Definindo $g=\sum_{i=1}^{n} f_{x_{i}} \overline{f_{x_{i}}}$, temos que $g$ é estritamente positiva em $K$, logo invertível em $C(K)$. Entretanto, $\varphi(g)=\sum_{i=1}^{n} \varphi\left(f_{x_{i}} \overline{f_{x_{i}}}\right)=0$, contradição.

Exemplo 2.3.4. A álgebra de disco $\mathcal{A}(\mathcal{D})$ é natural.

De fato, seja $\varphi \in M_{\mathcal{A}(\mathcal{D})}$. Seja $x=\varphi(i d)$, onde $i d(z)=z, \forall z \in \overline{\mathcal{D}}$. Pelo item 3 da Proposição 2.1.12, temos $\|\varphi\|=1$. Daí,

$$
|x|=|\varphi(i d)| \leq\|\varphi\|\|i d\|=1 \sup _{z \in \overline{\mathcal{D}}}|i d(z)|=1 .
$$

Para cada $n \in \mathbb{N}$, seja $q(z)=\sum_{i=1}^{n} a_{i} z^{i}$, onde $a_{i} \in \mathbb{C}, \forall i=1, \cdots, n$. Assim,

$$
\varphi(q)=\sum_{i=1}^{n} a_{i} \varphi(i d)^{i}=\sum_{i=1}^{n} a_{i} x^{i}=q(x) .
$$

Como toda função em $\mathcal{A}(\mathcal{D})$ pode ser aproximada por uma sequência de polinômios (Proposição 2.1.4), segue que para cada $f \in \mathcal{A}(\mathcal{D})$, existe uma sequência $\left(p_{n}\right)$ de polinômios tal que $\left\|p_{n}-f\right\|_{\overline{\mathcal{D}}} \rightarrow$ 0 . Em particular, $p_{n}(x) \rightarrow f(x)$. Além disso,

$$
\left|\varphi(f)-p_{n}(x)\right|=\left|\varphi(f)-\varphi\left(p_{n}\right)\right|=\left|\varphi\left(f-p_{n}\right)\right| \leq\|\varphi\|\left\|f-p_{n}\right\|_{\overline{\mathcal{D}}} \rightarrow 0 .
$$

Logo, $p_{n}(x) \rightarrow \varphi(f)$. Pela unicidade do limite, $\varphi(f)=f(x), \forall f \in \mathcal{A}(\mathcal{D})$, i.e $\varphi=\varphi_{x}$.

O próximo resultado apresenta condições para que o espectro de uma álgebra de funções $\mathcal{A}$ seja homeomorfo ao espectro de $\overline{\mathcal{A}}$. Demonstrado por Honary em [18], esse resultado foi utilizado para encontrar o espectro de uma importante subálgebra das álgebras de Dales-Davie que serão introduzidas no próximo capítulo.

Proposição 2.3.5. (Honary, [18]) Sejam $\mathcal{A}$ e $\mathcal{B}$ álgebras de Banach de funções em $K$ tais que

1. $\mathcal{A} \subset \mathcal{B}$.

2. $\exists C>0,\|f\|_{\mathcal{A}} \leq C\|f\|_{\mathcal{B}}, \forall f \in \mathcal{A}$.

3. B é natural.

Então, $\mathcal{M}_{\mathcal{A}} \cong \mathcal{M}_{\overline{\mathcal{A}}}$. Além disso, se $\overline{\mathcal{A}}=\overline{\mathcal{B}}$, então $\mathcal{A}$ é natural.

Demonstração. Pelo Teorema de Gelfand 2.1.19, pela Fórmula do Raio Espectral 2.1.9 - 2 e pelos itens 2 e 3, segue que:

$$
\begin{aligned}
\|\hat{f}\| & =r_{\mathcal{A}}(f)=\lim _{n \rightarrow \infty}\left\|f^{n}\right\|_{\mathcal{A}}^{1 / n} \leq \lim _{n \rightarrow \infty} C^{1 / n}\left\|f^{n}\right\|_{\mathcal{B}}^{1 / n} \\
& \leq \lim _{n \rightarrow \infty} C^{1 / n} \lim _{n \rightarrow \infty}\left\|f^{n}\right\|_{\mathcal{B}}^{1 / n}=r_{\mathcal{B}}(f)=\|\hat{f}\|_{\mathcal{M}_{\mathcal{B}}} \\
& \leq\|f\|_{K} .
\end{aligned}
$$

Daí, pelo Teorema 2.2.7, $\mathcal{M}_{\mathcal{A}} \cong \mathcal{M}_{\overline{\mathcal{A}}}$. Além disso, se $\overline{\mathcal{A}}=\overline{\mathcal{B}}$, temos

$$
\mathcal{A} \subset \mathcal{B} \subset \overline{\mathcal{A}} \Rightarrow \mathcal{M}_{\overline{\mathcal{A}}} \subset \mathcal{M}_{\mathcal{B}} \subset \mathcal{M}_{\mathcal{A}}
$$

Como $\mathcal{M}_{\mathcal{A}} \cong \mathcal{M}_{\overline{\mathcal{A}}}$ e $\mathcal{M}_{\mathcal{B}} \cong K$, segue que $\mathcal{M}_{\mathcal{A}} \cong K$. 
No que segue desta seção, vamos estudar os espectros de $P(K)$ e $R(K)$ quando $K \subset \mathbb{C}$ é compacto. Primeiramente, veremos que o espectro de $P(K)$ é homeomorfo a sua envoltória polinomial convexa que será definida a seguir:

Definição 2.3.6. Seja $K \subset \mathbb{C}$ limitado. Definimos a envoltória polinomial convexa de $K$ em $\mathbb{C}$ pelo conjunto

$$
\hat{K}=\left\{z \in \mathbb{C}:|p(z)| \leq \sup _{w \in K}|p(w)|, \forall p \in \mathbb{C}[z]\right\} .
$$

Além disso, $K$ é dito polinomialmente convexo se $K=\hat{K}$.

A envoltória polinomial convexa pode ser definida de modo mais geral em $\mathbb{C}^{n}$. Entretanto, abordaremos apenas o caso apresentado acima nesta dissertação. Para um estudo mais aprofundado sobre o tema, sugiro o capítulo 3 do livro [15].

Exemplos 2.3.7. $\quad$ 1. Um compacto $K \subset \mathbb{C}$ é polinomialmente convexo se, e só se, $\mathbb{C} \backslash K$ não é convexo ([15], cap. 3, Lema 1.3). Como consequência, temos que bolas fechadas em $\mathbb{C}$ são polinomialmente convexos.

2. O conjunto $S^{1}$ não é polinomialmente convexo, sua envoltória polinomial convexa é $\overline{\mathcal{D}}$.

De fato, como todo polinômio é uma função analítica, o princípio do módulo máximo garante que $\sup _{z \in \overline{\mathcal{D}}}|p(z)|=\sup _{z \in S^{1}}|p(z)|, \operatorname{logo} \hat{S}^{1}=\overline{\mathcal{D}}$.

O próximo Lema mostra que toda função em $P(K)$ pode ser estendida continuamente para uma função em $P(\hat{K})$.

Lema 2.3.8. Se $K \subset \mathbb{C}$ é compacto, então toda função em $P(K)$ pode ser estendida continuamente para $\hat{K}$.

Demonstração. Sejam $f \in P(K)$ e $\left(p_{n}\right) \subset \mathbb{C}[z]$ tal que $\left\|p_{n}-f\right\|_{K} \rightarrow 0$. Como, $\left(p_{n}\right)$ é sequência de Cauchy e $\left|\left(p_{n}-p_{m}\right)(z)\right| \leq\left\|p_{n}-p_{m}\right\|_{K}, \forall z \in \hat{K}$, temos que $\left\|p_{n}-p_{m}\right\|_{\hat{K}} \leq\left\|p_{n}-p_{m}\right\|_{K}$, isto é $\left(p_{n}\right)$ converge uniformemente em $\hat{K}$ para uma extensão contínua de $f$.

A seguir, apresentaremos a demonstração de que $\mathcal{M}_{P(K)} \cong \hat{K}$.

Proposição 2.3.9. Se $K \subset \mathbb{C}$ é compacto, então o espectro de $P(K)$ é $\hat{K}$.

Demonstração. Pelo Lema 2.3.8, se $z_{0} \in \hat{K}$, podemos definir $\varphi_{z_{0}}(f)=f\left(z_{0}\right), \forall f \in P(K)$. Assim, identificamos $\hat{K}$ como um subconjunto do espectro de $P(K)$.

Por outro lado, se $\varphi \in \mathcal{M}_{P(K)}$ e $z_{0}=\varphi(i d)$, temos

$$
\varphi(p)=\sum_{k=0}^{n} a_{k} \varphi(i d)^{k}=p\left(z_{0}\right), \forall p=\sum_{k=0}^{n} a_{k} z^{k} \in \mathbb{C}[z] .
$$

Assim, $\left|p\left(z_{0}\right)\right|=|\varphi(p)| \leq\|p\|_{X}, \forall p \in \mathbb{C}[z] \Rightarrow z_{0} \in \hat{K}$. Se $f \in P(K)$, existe sequência $\left(p_{n}\right)$ de polinômios tal que $\left\|p_{n}-f\right\|_{\hat{K}} \rightarrow 0$. Daí, $\varphi\left(p_{n}\right)=p_{n}\left(z_{0}\right) \rightarrow f\left(z_{0}\right)$ e $\varphi\left(p_{n}\right) \rightarrow \varphi(f)$. Portanto,

$$
\varphi(f)=f\left(z_{0}\right), \forall f \in P(K) \Rightarrow \mathcal{M}_{P(K)} \cong \hat{K} .
$$

Por continuidade, temos que $p_{n}\left(z_{0}\right)=\varphi\left(p_{n}\right) \rightarrow \varphi(f)$.

Vamos precisar do próximo Lema para estudar o espectro de $R(K)$.

Lema 2.3.10. Seja $K \subset \mathbb{C}$ compacto. Então, temos as seguintes igualdades de conjuntos:

$$
\left\{z \in \mathbb{C}:|r(z)| \leq\|r\|_{K}, \forall r \in R_{0}(K)\right\}=\{z \in \mathbb{C}: f(z) \in f(K), \forall f \in \mathbb{C}[z]\}=K .
$$


Demonstração. É imediato que $K \subset\left\{z \in \mathbb{C}:|r(z)| \leq\|r\|_{K}, \forall r \in R_{0}(K)\right\}$.

Veremos agora que

$$
\left\{z \in \mathbb{C}:|r(z)| \leq\|r\|_{K}, \forall r \in R_{0}(K)\right\} \subset\{z \in \mathbb{C}: f(z) \in f(K), \forall f \in \mathbb{C}[z]\} .
$$

De fato, seja $z_{0} \in \mathbb{C}$ tal que $f\left(z_{0}\right) \notin f(K)$ para algum $f \in \mathbb{C}[z]$. Daí, $r(z)=\frac{1}{f(z)-f\left(z_{0}\right)} \in R_{0}(K)$ e $+\infty=\lim _{z \rightarrow z_{0}}|r(z)|>\|r\|_{K}$.

Para concluirmos a demonstração, é suficiente provar que

$$
\{z \in \mathbb{C}: f(z) \in f(K), \forall f \in \mathbb{C}[z]\} \subset K .
$$

Com efeito, se $z_{0} \in \mathbb{C}$ é tal que $f(z) \in f(K), \forall f \in \mathbb{C}[z]$, temos, em particular, que $z_{0}=i d\left(z_{0}\right) \in$ $i d(K)=K$. Logo, $z_{0} \in K$.

Utilizando o Lema 2.3.10, vamos demonstrar que $\mathcal{M}_{R(K)} \cong K$, i.e $R(K)$ é natural.

Proposição 2.3.11. Seja $K \subset \mathbb{C}$ compacto. Então, o espectro de $R(K)$ é homeomorfo ao conjunto $K$.

Demonstração. Dado $\varphi \in \mathcal{M}_{R(K)}$, seja $z_{0}=\varphi(i d)$. Vimos na demonstração da Proposição 2.3.9 que se $p \in \mathbb{C}[z]$, temos $\varphi(p)=p\left(z_{0}\right)$. Assim, se $r=p / q \in R_{0}(K)$, segue que

$$
\varphi(r)=\varphi(p / q)=\varphi(p) / \varphi(q)=p\left(z_{0}\right) / q\left(z_{0}\right)=r\left(z_{0}\right) .
$$

Daí, $\left|r\left(z_{0}\right)\right|=|\varphi(r)| \leq\|r\|_{K}, \forall r \in R_{0}(K) \Rightarrow z_{0} \in K$ (Lema 2.3.10).

Desse modo, se $f \in R(K)$, existe $\left(r_{n}\right) \subset R_{0}(K),\left\|r_{n}-f\right\|_{K} \rightarrow 0$. Portanto, $\varphi\left(r_{n}\right) \rightarrow \varphi(f)$ e $r_{n}\left(z_{0}\right)=\varphi\left(r_{n}\right) \rightarrow f\left(z_{0}\right)$. Pela unicidade do limite, concluímos que $\varphi(f)=f\left(z_{0}\right), \forall f \in R(K)$. Logo, $\mathcal{M}_{R(K)} \cong K$. 


\section{Capítulo 3}

\section{Álgebras de Dales-Davie}

O objetivo deste trabalho é estudar certas álgebras de funções infinitamente deriváveis. Essas álgebras foram introduzidas por Dales e Davie em [11] e, posteriormente, denominadas de álgebras de Dales-Davie por Abtahi e Honary em [2]. Tais álgebras podem ser consideradas como uma extensão das álgebras $D^{n}(X)$ e $D^{\infty}(X)$ definidas em 2.2.3.

Recordemos que $D^{n}(X)$ é a álgebra das funções $f: X \rightarrow \mathbb{C}$ com $n$-ésima derivada contínua em $X$ munida da norma

$$
\|f\|_{n}=\sum_{k=0}^{n} \frac{1}{k !}\left\|f^{(k)}\right\|_{X} .
$$

No entanto, se $f: X \rightarrow \mathbb{C}$ é infinitamente derivável em $X$, isto é $f \in D^{\infty}(X)$, a série $\sum_{k=0}^{\infty} \frac{1}{k !}\left\|f^{(k)}\right\|_{X}$ não é necessariamente convergente. Uma pergunta natural é se o espaço vetorial

$$
\left\{f \in D^{\infty}(X): \sum_{k=0}^{\infty} \frac{1}{k !}\left\|f^{(k)}\right\|_{X}<\infty\right\}
$$

é uma álgebra normada de funções em $X$ com a norma $\|f\|=\sum_{k=0}^{\infty} \frac{1}{k !}\left\|f^{(k)}\right\|_{X}<\infty$. Em [11], Dales e Davie mostraram um resultado mais forte como veremos neste capítulo.

\subsection{Introdução}

Nesta seção, apresentaremos a definição dessas álgebras e mostraremos que são de fato álgebras normadas. Primeiramente, vamos definir as sequências de álgebras:

Definição 3.1.1. Uma sequência $\left(M_{n}\right)$ de números reais positivos é dita sequência de álgebra se satisfizer

$$
M_{0}=1, \quad \frac{M_{n}}{M_{k} M_{n-k}} \geq\left(\begin{array}{l}
n \\
k
\end{array}\right), \forall k=0,1, \cdots, n, \forall n \in \mathbb{N} .
$$

A seguir veremos alguns exemplos.

Exemplos 3.1.2. 1. Sejam $M_{0}=1$ e $M_{n}=\alpha n$ !, onde $0<\alpha \leq 1$. Então, $M=\left(M_{n}\right)$ é uma sequência de álgebra. De fato,

$$
\frac{M_{n}}{M_{n-k} M_{k}}=\frac{1}{\alpha}\left(\begin{array}{l}
n \\
k
\end{array}\right) \geq\left(\begin{array}{l}
n \\
k
\end{array}\right) .
$$

2. $M_{0}=1, M_{n}=n !^{\alpha}, \alpha>1$, define uma sequência de álgebra.

$$
\frac{M_{n}}{M_{n-k} M_{k}}=\frac{n !^{\alpha}}{(n-k) !^{\alpha} k !^{\alpha}} \geq\left(\begin{array}{l}
n \\
k
\end{array}\right)^{\alpha} \geq\left(\begin{array}{l}
n \\
k
\end{array}\right) .
$$


Usando as sequências de álgebra definidas em 3.1.1, Dales e Davie puderam generalizar o conjunto 3.1 como veremos no próximo Teorema.

Teorema 3.1.3. Sejam $X \subset \mathbb{C}$ compacto e perfeito e $\left(M_{n}\right)$ uma sequência de álgebra. Então,

$$
\mathcal{A}=\left\{f \in D^{\infty}(X): \sum_{n=0}^{\infty} \frac{1}{M_{n}}\left\|f^{(n)}\right\|_{X}<\infty\right\}
$$

é uma álgebra de funçôes em $X$. Além disso, $\|f\|=\sum_{n=0}^{\infty} \frac{1}{M_{n}}\left\|f^{(n)}\right\|_{X}$ define uma norma em $\mathcal{A}$.

Demonstração. Note que $\mathcal{A}$ separa os pontos de $X$ e contém as constantes, pois os polinômios estão contidos em $\mathcal{A}$. Dados $f, g \in \mathcal{A}$ e $n \in \mathbb{N}$, temos:

$$
\begin{aligned}
\sum_{k=0}^{n} \frac{1}{M_{k}}\left\|(f+g)^{(k)}\right\|_{X} & =\sum_{k=0}^{n} \frac{1}{M_{k}}\left\|f^{(k)}+g^{(k)}\right\|_{X} \\
& \leq \sum_{k=0}^{n} \frac{1}{M_{k}}\left[\left\|f^{(k)}\right\|_{X}+\left\|g^{(k)}\right\|_{X}\right] \\
& \leq \sum_{k=0}^{\infty} \frac{1}{M_{k}}\left\|f^{(k)}\right\|_{X}+\sum_{k=0}^{\infty} \frac{1}{M_{k}}\left\|g^{(k)}\right\|_{X} \\
& =\|f\|+\|g\|<\infty .
\end{aligned}
$$

Fazendo $n \rightarrow \infty$, segue que $\sum_{k=0}^{\infty} \frac{1}{M_{k}}\left\|(f+g)^{(k)}\right\|_{X}<\infty$, o que implica que $f+g \in \mathcal{A}$. Em particular, $\|f+g\| \leq\|f\|+\|g\|$.

Agora, dados $f \in \mathcal{A}, \lambda \in \mathbb{C}$ e $n \in \mathbb{N}$ :

$$
\begin{aligned}
\sum_{k=0}^{n} \frac{1}{M_{k}}\left\|(\lambda f)^{(k)}\right\|_{X} & =\sum_{k=0}^{n} \frac{1}{M_{k}}\left\|\lambda f^{(k)}\right\|_{X} \\
& =\sum_{k=0}^{n} \frac{|\lambda|}{M_{k}}\left\|f^{(k)}\right\|_{X} \\
& =|\lambda| \sum_{k=0}^{n} \frac{1}{M_{k}}\left\|f^{(k)}\right\|_{X} \\
& \leq|\lambda| \sum_{k=0}^{\infty} \frac{1}{M_{k}}\left\|f^{(k)}\right\|_{X} \\
& \leq|\lambda|\|f\|<\infty .
\end{aligned}
$$

Fazendo $n \rightarrow \infty$, temos $\sum_{k=0}^{\infty} \frac{1}{M_{k}}\left|(\lambda f)^{(k)}\right|_{X}<\infty$, implicando $\lambda f \in \mathcal{A}$ Daí,

$$
\|\lambda f\|=\sum_{k=0}^{\infty} \frac{1}{M_{k}}\left|(\lambda f)^{(k)}\right|_{X}=\lambda \sum_{k=0}^{\infty} \frac{1}{M_{k}}\left|f^{(k)}\right|_{X}=\lambda\|f\| .
$$


Por fim, dados $f, g \in \mathcal{A}$ e $n \in \mathbb{N}$, segue que

$$
\begin{aligned}
\sum_{k=0}^{n} \frac{1}{M_{k}}\left\|(f g)^{(k)}\right\|_{X} & =\sum_{k=0}^{n} \frac{1}{M_{k}}\left\|\sum_{j=0}^{k}\left(\begin{array}{c}
k \\
j
\end{array}\right) f^{(j)} g^{(k-j)}\right\|_{X} \\
& \leq \sum_{k=0}^{n} \frac{1}{M_{k}} \sum_{j=0}^{k}\left(\begin{array}{c}
k \\
j
\end{array}\right)\left\|f^{(j)}\right\|_{X}\left\|g^{(k-j)}\right\|_{X} \\
& \leq \sum_{k=0}^{(i)} \frac{1}{M_{k}} \sum_{j=0}^{k} \frac{M_{k}}{M_{j} M_{k-j}}\left\|f^{(j)}\right\|_{X}\left\|g^{(k-j)}\right\|_{X} \\
& \leq \sum_{k=0}^{n} \sum_{j=0}^{k} \frac{1}{M_{j} M_{k-j}}\left\|f^{(j)}\right\|_{X}\left\|g^{(k-j)}\right\|_{X} .
\end{aligned}
$$

Em (i), usamos a desigualdade 3.2. Mas como

$$
\begin{aligned}
\sum_{k=0}^{n} \sum_{j=0}^{k} \frac{1}{M_{j} M_{k-j}}\left\|f^{(j)}\right\|_{X}\left\|g^{(k-j)}\right\|_{X} & =\frac{\left\|f^{(0)}\right\|_{X}}{M_{0}}\left(\frac{\left\|g^{(0)}\right\|_{X}}{M_{0}}+\frac{\left\|g^{(1)}\right\|_{X}}{M_{1}}+\cdots+\frac{\left\|g^{(n)}\right\|_{X}}{M_{n}}\right)+ \\
& +\frac{\left\|f^{(1)}\right\|_{X}}{M_{1}}\left(\frac{\left\|g^{(0)}\right\|_{X}}{M_{0}}+\frac{\left\|g^{(1)}\right\|_{X}}{M_{1}}+\cdots+\frac{\left\|g^{(n-1)}\right\|_{X}}{M_{n-1}}\right)+ \\
& +\cdots+\frac{\left\|f^{(n)}\right\|_{X}}{M_{n}}\left(\frac{\left\|g^{(0)}\right\|_{X}}{M_{0}}\right) \\
& \leq\left(\sum_{j=0}^{n} \frac{\left\|f^{(j)}\right\|_{X}}{M_{j}}\right)\left(\sum_{l=0}^{n} \frac{\left\|g^{(l)}\right\|_{X}}{M_{l}}\right) \\
& \leq\left(\sum_{j=0}^{\infty} \frac{\left\|f^{(j)}\right\|_{X}}{M_{j}}\right)\left(\sum_{l=0}^{\infty} \frac{\left\|g^{(l)}\right\|_{X}}{M_{l}}\right)=\|f\|\|g\|<\infty
\end{aligned}
$$

podemos concluir que:

$$
\sum_{k=0}^{n} \frac{1}{M_{k}}\left\|(f g)^{(k)}\right\|_{X} \leq\|f\|\|g\| .
$$

Para $n \rightarrow \infty, \sum_{k=0}^{\infty} \frac{1}{M_{k}}\left|(f g)^{(k)}\right|_{X}<\infty$, e concluímos que $f g \in \mathcal{A}$ e $\|f g\| \leq\|f\|\|g\|$.

Pelo feito acima, segue que $\mathcal{A}$ é uma álgebra. Ademais, concluímos que $\|f+g\| \leq\|f\|+$ $\|g\|,\|\lambda f\|=\lambda\|f\|$ e $\|f g\| \leq\|f\|\|g\|$. Ainda falta verificar que $\|f\|=0 \Longleftrightarrow f=0$. Com efeito,

$$
0=\|f\|=\sum_{k=0}^{\infty} \frac{1}{M_{k}}\left\|f^{(k)}\right\|_{X} \Longleftrightarrow\left\|f^{(k)}\right\|_{X}=0, \forall k \in \mathbb{N} \Longleftrightarrow f=0 .
$$

Portanto, $\mathcal{A}$ é uma álgebra normada.

Tendo em vista, o Teorema 3.1.3, podemos definir as Álgebras de Dales-Davie.

Definição 3.1.4. Sejam $X \subset \mathbb{C}$ um compacto perfeito e $M=\left(M_{n}\right)$ uma sequência de álgebra. A álgebra

$$
\mathcal{A}=\left\{f \in D^{\infty}(X): \sum_{n=0}^{\infty} \frac{1}{M_{n}}\left\|f^{(n)}\right\|_{X}<\infty\right\}
$$


normada com $\|f\|=\sum_{n=0}^{\infty} \frac{1}{M_{n}}\left\|f^{(n)}\right\|_{X}$, será chamada de Álgebra de Dales-Davie e doravante denotada por $D(X, M)$.

Uma vez que $(n !)_{n \in \mathbb{N}_{0}}$ é uma sequência de álgebra, temos que 3.1 é um caso particular das álgebras de Dales-Davie. O próximo resultado apresenta uma sequência de inclusões entre $D(X, M)$ e as álgebras definidas em 2.2.3.

Note que se $X \subset \mathbb{C}$ é compacto e perfeito e se $M=\left(M_{n}\right)$ é uma sequência de álgebra, temos as seguintes inclusões:

$$
D(X, M) \subset D^{\infty}(X) \subset D^{n}(X) \subset D^{1}(X) .
$$

\subsection{Completude em $D(X, M)$}

Os resultados estudados nesta seção fazem parte dos artigos [7, 11, 19]. Nesta seção, vamos estudar a completude das Álgebras de Dales-Davie. Cronologicamente, em 1973, Dales e Davie apresentaram condições a respeito do conjunto $X$ que garantem que $D(X, M)$ seja uma álgebra de Banach. Mais precisamente, Dales e Davie mostraram que se $X$ se escreve como união finita de conjuntos uniformemente regulares, a álgebra será Banach [11].

De modo geral, essas álgebras não são completas. Em 2005, Bland e Feinstein mostraram que se $X \subset \mathbb{C}$ é compacto, perfeito e possui uma quantidade infinita de componentes conexas, então $D(X, M)$ não é completa [7].

No que segue, salvo menção contrária, $X$ denotará um subconjunto compacto e perfeito de $\mathbb{C}$ e $M=\left(M_{n}\right)$, uma sequência de álgebra. Iniciaremos a seção apresentando a demonstração de um resultado de Dales e Davie [11]. A partir deste, Dales e Davie reduziram o estudo da completude de $D(X, M)$ para estudar a completude em $D^{1}(X)$. Recordemos que em 2.2.3, consideramos em $D^{1}(X)$ a norma

$$
\|f\|_{1}=\|f\|_{X}+\left\|f^{\prime}\right\|_{X}, \forall f \in D^{1}(X) .
$$

Teorema 3.2.1. (Dales-Davie, [11]) Se $D^{1}(X)$ é álgebra de Banach, então $D(X, M)$ também o é. Demonstração. Seja $\left(f_{n}\right) \subset D(X, M)$ sequência de Cauchy. Dados $k \in \mathbb{N}_{0}$ e $\epsilon>0$, existe $n_{k} \in \mathbb{N}$ tal que $\forall n, m \geq n_{k}$, temos

$$
\left\|f_{n}-f_{m}\right\|=\sum_{j=0}^{\infty} \frac{1}{M_{j}}\left\|f_{n}^{(j)}-f_{m}^{(j)}\right\|_{X}<\frac{\epsilon}{2 M_{k}} .
$$

Em particular,

$$
\left\|f_{n}^{(k)}-f_{m}^{(k)}\right\|_{X}<\frac{\epsilon}{2}, \forall n, m \geq n_{k} .
$$

Para cada $k \in \mathbb{N}_{0}$, seja $M_{k}=\max \left\{n_{k}, n_{k+1}\right\}$. Assim,

$$
\left\|f_{n}^{(k)}-f_{m}^{(k)}\right\|_{X}+\left\|f_{n}^{(k+1)}-f_{m}^{(k+1)}\right\|_{X}<\epsilon, \forall n, m \geq M_{k} .
$$

Isto é, para todo $k \in \mathbb{N}_{0}\left(f_{n}^{(k)}\right)$ é sequência de Cauchy em $D^{1}(X)$. Como $D^{1}(X)$ é álgebra de Banach, para cada $k \geq 0$, existe $g_{k} \in D^{1}(X)$ tal que

$$
\left\|f_{n}^{(k)}-g_{k}\right\|_{1}=\left\|f_{n}^{(k)}-g_{k}\right\|_{X}+\left\|f_{n}^{(k+1)}-g_{k}^{\prime}\right\|_{X} \rightarrow 0 .
$$

Em particular, $\left(f_{n}^{(k+1)}\right)$ converge uniformemente para $g_{k+1}$ em $X, \operatorname{logo}, g_{k}^{\prime}=g_{k+1}, \forall k \geq 0$. Portanto, $f_{n}$ converge uniformemente para $g_{0}$ e $f_{n}^{(k)}$ converge uniformemente para $g_{0}^{(k)}$.

Resta verificar que $g_{0} \in D(X, M)$ e que $\left(f_{n}\right)$ converge para $g_{0}$ em $D(X, M)$. Com efeito, se $\sum_{k=0}^{\infty} \frac{1}{M_{k}}\left\|f_{n}^{(k)}-g_{0}^{(k)}\right\|_{X}=+\infty$, então, para cada $L>0$, existe $K_{L} \in \mathbb{N}_{0}$ tal que

$$
\sum_{k=0}^{K_{L}} \frac{1}{M_{k}}\left\|f_{n}^{(k)}-g_{0}^{(k)}\right\|_{X}>L, \quad \forall n \in \mathbb{N} .
$$


Por outro lado, como $\left\|f_{n}^{(k)}-g_{0}^{(k)}\right\|_{X} \rightarrow 0$ quando $n \rightarrow \infty$. Para cada $k=0,1, \cdots, K_{L}$, existe $n_{k} \in \mathbb{N}_{0}$ tal que

$$
\left\|f_{n}^{(k)}-g_{0}^{(k)}\right\|_{X}<\frac{1}{\sum_{j=0}^{K_{L}} 1 / M_{j}} L, \forall n \geq n_{k}
$$

Desse modo, se $\bar{N}=\max \left\{n_{0}, n_{1}, \cdots, n_{K_{L}}\right\}$, temos

$$
\sum_{k=0}^{K_{L}} \frac{1}{M_{k}}\left\|f_{n}^{(k)}-g_{0}^{(k)}\right\|_{X} \leq\left(\sum_{k=0}^{K_{L}} \frac{1}{M_{k}}\right) \frac{1}{\sum_{j=0}^{K_{L}} 1 / M_{j}} L=L,
$$

contradição. Portanto, $\sum_{k=0}^{\infty} \frac{1}{M_{k}}\left\|f_{n}^{(k)}-g_{0}^{(k)}\right\|_{X}<\infty$.

Em particular, existe $N \in \mathbb{N}$ tal que $f_{N}-g_{0} \in D(X, M)$, como $D(X, M)$ é álgebra e $f_{N} \in$ $D(X, M), g_{0} \in D(X, M)$. Além disso, $\left\|f_{n}-g_{0}\right\| \rightarrow 0$ quando $n \rightarrow \infty$. Desse modo, concluímos que $D(X, M)$ é álgebra de Banach.

A partir do Teorema 3.2.1, se $D^{1}(X)$ é Banach, então $D(X, M)$ também o é. Desse modo, Dales e Davie apresentaram um condição suficiente para garantir a completude de $D^{1}(X)$ [11].

Teorema 3.2.2. (Dales-Davie [11]) Suponha que para todo $z_{0} \in X$, existe $C_{0}>0$ tal que

$$
\left|f(z)-f\left(z_{0}\right)\right| \leq C_{0}\left|z-z_{0}\right|\left(\|f\|_{X}+\left\|f^{\prime}\right\|_{X}\right), \forall z \in X, \forall f \in D^{1}(X) .
$$

Então, $D^{1}(X)$ é Banach.

Demonstração. Seja $\left(f_{n}\right)$ sequência de Cauchy em $D^{1}(X)$ com a norma $\|\cdot\|_{1}$. Então, dado $\epsilon>0$, existe $n_{0} \in \mathbb{N}$ tal que

$$
\left\|f_{n}-f_{m}\right\|_{1}=\left\|f_{n}-f_{m}\right\|_{X}+\left\|f_{n}^{\prime}-f_{m}^{\prime}\right\|_{X}<\epsilon, \forall n, m \geq n_{0} .
$$

Portanto, $\left(f_{n}\right)$ e $\left(f_{n}^{\prime}\right)$ são sequências de Cauchy em $C(X)$. Desso modo, existem $f, g \in C(X)$ tais que $\left\|f_{n}-f\right\|_{X} \rightarrow 0$ e $\left\|f_{n}^{\prime}-g\right\|_{X} \rightarrow 0$ em $C(X)$. Dados $z_{0} \in X, \epsilon>0$ e $h \neq 0$, tome $\delta=\epsilon|h| \frac{1}{2 C_{0}\left(\|f\|_{X}+\|g\|_{X}\right)}$. Se $\left|z-z_{0}\right|<\delta \mathrm{e}$

$$
\star=\left|\frac{f(z+h)-f(z)}{h}-\frac{f\left(z_{0}+h\right)-f\left(z_{0}\right)}{h}\right|,
$$

temos

$$
\begin{aligned}
\star & =\frac{1}{|h|}\left|\lim \left(f_{n}(z+h)-f_{n}(z)\right)-\lim \left(f_{n}\left(z_{0}+h\right)-f_{n}\left(z_{0}\right)\right)\right| \\
& \leq \frac{1}{|h|} \lim \left|f_{n}(z+h)-f_{n}(z)-\left(f_{n}\left(z_{0}+h\right)-f_{n}\left(z_{0}\right)\right)\right| \\
& \leq \frac{1}{|h|} \lim \left[\left|f_{n}(z+h)-f_{n}\left(z_{0}+h\right)\right|+\left|f_{n}(z)-f_{n}\left(z_{0}\right)\right|\right] .
\end{aligned}
$$

Aplicando a hipótese do Teorema, segue que

$$
\begin{aligned}
\star & \leq \frac{1}{|h|} \lim 2 C_{0}\left|z-z_{0}\right|\left(\left\|f_{n}\right\|_{X}+\left\|f_{n}^{\prime}\right\|_{X}\right) \\
& \leq \frac{2 C_{0}}{|h|}\left|z-z_{0}\right|\left(\lim \left\|f_{n}\right\|_{X}+\lim \left\|f_{n}^{\prime}\right\|_{X}\right) .
\end{aligned}
$$

Pela continuidade da norma e a convergência uniforme de $f_{n}$ e $f_{n}^{\prime}$ para $f$ e $g$, obtemos 


$$
\begin{gathered}
\star \\
\quad \leq \frac{2 C_{0}}{|h|}\left|z-z_{0}\right|\left(\|f\|_{X}+\|g\|_{X}\right) \\
<\frac{2 C_{0}}{|h|} \delta\left(\|f\|_{X}+\|g\|_{X}\right)=\epsilon
\end{gathered}
$$

Desse modo,

$$
\left|f^{\prime}(z)-f^{\prime}\left(z_{0}\right)\right| \leq \lim _{h \rightarrow 0}\left|\frac{f(z+h)-f(z)}{h}-\frac{f\left(z_{0}+h\right)-f\left(z_{0}\right)}{h}\right|<\epsilon .
$$

Portanto, $f^{\prime}$ é contínua em $z_{0} \in X$. Além disso,

$$
\begin{aligned}
f^{\prime}\left(z_{0}\right) & =\lim _{z \rightarrow z_{0}} \frac{f(z)-f\left(z_{0}\right)}{z-z_{0}}=\lim _{z \rightarrow z_{0}} \lim _{n \rightarrow \infty} \frac{f_{n}(z)-f_{n}\left(z_{0}\right)}{z-z_{0}} \\
& =\lim _{n \rightarrow \infty} \lim _{z \rightarrow z_{0}} \frac{f_{n}(z)-f_{n}\left(z_{0}\right)}{z-z_{0}}=\lim _{n \rightarrow \infty} f_{n}^{\prime}\left(z_{0}\right) \\
& =g\left(z_{0}\right) .
\end{aligned}
$$

Como $z_{0} \in X$ é arbitrário, $f^{\prime}=g \in C(X)$. Logo, $f \in D^{1}(X)$. Mas como $\left\|f_{n}-f\right\|_{X} \rightarrow 0$ e $\left\|f_{n}^{\prime}-f^{\prime}\right\|_{X} \rightarrow 0$, segue que $\left\|f_{n}-f\right\|_{1} \rightarrow 0$.

Aproximadamente 26 anos depois, Honary e Mahyar mostraram a recíproca do Teorema 3.2.2 utilizando o Teorema da Aplicação Aberta como veremos a seguir.

Teorema 3.2.3. Se $D^{1}(X)$ é Banach com a norma $\|\cdot\|_{1}$, então para cada $z_{0} \in X$, existe $C_{0}>0$ tal que

$$
\left|f(z)-f\left(z_{0}\right)\right| \leq C_{0}\left|z-z_{0}\right|\left(\|f\|_{X}+\left\|f^{\prime}\right\|_{X}\right), \forall z \in X, \forall f \in D^{1}(X) .
$$

Demonstração. Dado $z_{0} \in X$, não é difícil verificar que

$$
\|f\|_{0}=\|f\|_{X}+\left\|f^{\prime}\right\|_{X}+\sup _{z \neq z_{0}} \frac{\left|f\left(z_{0}\right)-f(z)\right|}{\left|z-z_{0}\right|}, \forall f \in D^{1}(X)
$$

define uma norma no espaço $D^{1}(X)$. Por definição, temos que $\|f\|_{0} \leq\|f\|_{1}, \forall f \in D^{1}(X)$. Desse modo, o operador identidade

$$
i:\left(D^{1}(X),\|\cdot\|_{1}\right) \rightarrow\left(D^{1}(X),\|\cdot\|_{0}\right)
$$

é uma bijeção linear contínua entre espaços normados.

Vamos mostrar a partir do Teorema da Aplicação Aberta 1.3.2 que a inversa também é contínua. Desse modo, como $\left(D^{1}(X),\|\cdot\|_{1}\right)$ é Banach, é suficiente verificar que $\left(D^{1}(X),\|\cdot\|_{0}\right)$ também o é.

Com efeito, seja $\left(f_{n}\right)$ uma sequência em $D^{1}(X)$ de $\|\cdot\|_{0}$-Cauchy. Então, dado $\epsilon>0$, existe $n_{0} \in \mathbb{N}$ tal que para todos $n, m \geq n_{0},\left\|f_{n}-f_{m}\right\|_{0}<\epsilon$. Em particular,

$$
\left\|f_{n}-f_{m}\right\|_{1}=\left\|f_{n}-f_{m}\right\|_{X}+\left\|f_{n}^{\prime}-f_{m}^{\prime}\right\|_{X}<\epsilon, \forall n, m \geq n_{0},
$$

isto é $\left(f_{n}\right)$ é $\|\cdot\|_{1}$-Cauchy. Mas como $D^{1}(X)$ é Banach com a norma $\|\cdot\|_{1}$, existe $f \in D^{1}(X)$ tal que $\left\|f_{n}-f\right\|_{1} \rightarrow 0$. Desse modo, $f_{n}$ converge uniformemente para $f$ em $X$. Daí, se $z \in X$ tal que $z \neq z_{0}$,

$$
\begin{aligned}
\frac{\left|\left(f_{n}(z)-f(z)\right)-\left(f_{n}\left(z_{0}\right)-f\left(z_{0}\right)\right)\right|}{\left|z-z_{0}\right|} & \leq \frac{\left|f_{n}(z)-f(z)\right|+\left|f_{n}\left(z_{0}\right)-f\left(z_{0}\right)\right|}{\left|z-z_{0}\right|} \\
& \leq \frac{2\left\|f_{n}-f\right\|_{X}}{\left|z-z_{0}\right|} \rightarrow 0 .
\end{aligned}
$$


Daí,

$$
\sup _{z \neq z_{0}} \frac{\left|\left(f_{n}(z)-f(z)\right)-\left(f_{n}\left(z_{0}\right)-f\left(z_{0}\right)\right)\right|}{\left|z-z_{0}\right|} \rightarrow 0 .
$$

Por conseguinte,

$$
\left\|f_{n}-f\right\|_{0}=\left\|f_{n}-f\right\|_{1}+\sup _{z \neq z_{0}} \frac{\left|\left(f_{n}(z)-f(z)\right)-\left(f_{n}\left(z_{0}\right)-f\left(z_{0}\right)\right)\right|}{\left|z-z_{0}\right|} \rightarrow 0 .
$$

Logo, $D^{1}(X)$ é espaço de Banach com a norma $\|\cdot\|_{0}$. Assim, temos que $i^{-1}:\left(D^{1}(X),\|\cdot\|_{0}\right) \rightarrow$ $\left(D^{1}(X),\|\cdot\|_{1}\right)$ é um operador contínuo. Então, existe $C_{0}>0$ tal que $\|f\|_{0} \leq C_{0}\|f\|_{1}, \forall f \in D^{1}(X)$. Isto é,

$$
\|f\|_{1}+\sup _{z \neq z_{0}} \frac{\left|f(z)-f\left(z_{0}\right)\right|}{\left|z-z_{0}\right|} \leq C_{0}\|f\|_{1} .
$$

Donde,

$$
\sup _{z \neq z_{0}} \frac{\left|f(z)-f\left(z_{0}\right)\right|}{\left|z-z_{0}\right|} \leq C_{0}\|f\|_{1}
$$

Portanto, $\left|f(z)-f\left(z_{0}\right)\right| \leq C_{0}\left|z-z_{0}\right|\|f\|_{1}, \forall z \in X, \forall f \in D^{1}(X)$.

O Lema 3.2.4 que será apresentado a seguir foi demonstrado por Dales e Davie em [11]. Dales e Davie o utilizaram para provar dois resultados importantes que serão apresentados nesta dissertação, que são os Teoremas 3.2.5 e 3.3.2 deste texto.

Lema 3.2.4. (Dales-Davie, [11]) Sejam $X$ um conjunto uniformemente regular e $z_{0} \in X$, então existe $C>0$ tal que

$$
\left|f(z)-f\left(z_{0}\right)\right| \leq \delta\left(z, z_{0}\right)\left\|f^{\prime}\right\|_{X} \leq C\left|z-z_{0}\right|\left\|f^{\prime}\right\|_{X}, \forall f \in D^{1}(X), \forall z \in X .
$$

Demonstração. A segunda desigualdade é imediata da definição de conjunto uniformemente regular. Vamos provar, pois, a primeira. Sejam $z \in X$ e $\alpha$ a curva em $X$ de comprimento $\delta\left(z, z_{0}\right)$ que liga $z$ e $z_{0}$. Para cada $f \in D^{1}(X)$ e para cada $x \in \alpha([0,1])$, defina a função $h_{x}: X \rightarrow \mathbb{C}$ por $h_{x}(y)=\left|\frac{f(x)-f(y)}{x-y}\right|$. Daí, $\lim _{y \rightarrow x} h_{x}(y)=\left|f^{\prime}(x)\right|$. Portanto, fixados $f \in D^{1}(X)$ e $x \in \alpha([0,1])$, dado $\epsilon>0$, existe $\delta_{x}>0$ tal que se $y \in X$ e $0<|x-y|<\delta_{x}$, temos $h_{x}(y) \leq\left|f^{\prime}(x)\right|+\epsilon$, i.e

$$
\frac{|f(x)-f(y)|}{|x-y|} \leq\left|f^{\prime}(x)\right|+\epsilon \leq\left\|f^{\prime}\right\|_{\alpha([0,1])}+\epsilon .
$$

Logo, $|f(x)-f(y)| \leq|x-y|\left(\left\|f^{\prime}\right\|_{\alpha([0,1])}+\epsilon\right), \forall y \in U_{x}=B\left(x, \delta_{x}\right) \cap X$.

Em particular, $\left\{B\left(x, \delta_{x}\right): x \in \alpha([0,1])\right\}$ é cobertura aberta para $\alpha([0,1])$ que é compacto. Então, existem $z_{1}, \ldots, z_{m} \in \alpha([0,1])$ de modo que se $z_{m+1}=z, \alpha([0,1]) \subset \bigcup_{i=0}^{m+1} B\left(z_{i}, \delta_{z_{i}}\right) \mathrm{e}$

$$
\left|f\left(z_{i+1}\right)-f\left(z_{i}\right)\right| \leq\left|z_{i+1}-z_{i}\right|\left(\left\|f^{\prime}\right\|_{\alpha([0,1])}+\epsilon\right), \forall i=0,1, \ldots, m .
$$


Daí,

$$
\begin{aligned}
\left|f(z)-f\left(z_{0}\right)\right| & \leq \sum_{i=0}^{m}\left|f\left(z_{i+1}\right)-f\left(z_{i}\right)\right| \\
& \leq \sum_{i=0}^{m}\left|z_{i+1}-z_{i}\right|\left(\left\|f^{\prime}\right\|_{\alpha([0,1])}+\epsilon\right) \\
& \leq \sum_{i=0}^{m} \delta\left(z_{i+1}, z_{i}\right)\left(\left\|f^{\prime}\right\|_{\alpha([0,1])}+\epsilon\right) \\
& \leq \delta\left(z, z_{0}\right)\left(\left\|f^{\prime}\right\|_{\alpha([0,1])}+\epsilon\right) \\
& \leq \delta\left(z, z_{0}\right)\left(\left\|f^{\prime}\right\|_{X}+\epsilon\right) .
\end{aligned}
$$

Como $\epsilon>0$ é arbitrário, tomando $\epsilon \rightarrow 0$, temos

$$
\left|f(z)-f\left(z_{0}\right)\right| \leq \delta\left(z, z_{0}\right)\left\|f^{\prime}\right\|_{X} .
$$

A partir do Lema 3.2.4, Dales e Davie mostraram que se $X$ se escreve como união finita de conjuntos uniformemente regulares, então $D^{1}(X)$ será uma álgebra de Banach, como veremos a seguir.

Teorema 3.2.5. (Dales-Davie, [11]) Se $X \subset \mathbb{C}$ for união finita de conjuntos uniformemente regulares, então $D^{1}(X)$ é álgebra de Banach. Consequentemente, $D(X, M)$ também o é.

Demonstração. É suficiente provar a desigualdade do enunciado do Teorema 3.2.2. Com efeito, escreva $X$ como união disjunta de conjuntos uniformemente regulares $A_{1}, \cdots, A_{N}$ - podemos supor dessa maneira, pois a união não disjunta de dois conjuntos uniformemente regulares é conjunto uniformemente regular (Lema 1.2.17).

Dado $z_{0} \in X$, escreva $X=X_{1} \cup X_{2}$, onde $X_{1}$ é a componente uniformemente regular de $X$ que contém $z_{0}$ e $X_{2}=X \backslash X_{1}$. Pelo Lema 3.2.4, existe $C_{1}>0$ tal que

$$
\left|f(z)-f\left(z_{0}\right)\right| \leq C_{1}\left|z-z_{0}\right|\left\|f^{\prime}\right\|_{X_{1}}, \forall f \in D^{1}\left(X_{1}\right), \forall z \in X_{1} .
$$

Como $X_{1} \subset X$, se $f \in D^{1}(X)$, temos $f \in D^{1}\left(X_{1}\right)$ e $\left\|f^{\prime}\right\|_{X_{1}} \leq\left\|f^{\prime}\right\|_{X}$. Assim,

$$
\left|f(z)-f\left(z_{0}\right)\right| \leq C_{1}\left|z-z_{0}\right|\left\|f^{\prime}\right\|_{X}, \forall f \in D^{1}(X), \forall z \in X_{1} .
$$

Defina $C=C_{1}+2 \sup _{z \in X_{2}} \frac{1}{\left|z-z_{0}\right|}$, daí $\forall z \in X_{1}$,

$$
\left|f(z)-f\left(z_{0}\right)\right| \leq C\left|z-z_{0}\right|\left(\|f\|_{X}+\left\|f^{\prime}\right\|_{X}\right), \forall f \in D^{1}(X) .
$$

Por outro lado, se $z \in X_{2}$,

$$
\begin{aligned}
\left|f(z)-f\left(z_{0}\right)\right| & \leq|f(z)|+\left|f\left(z_{0}\right)\right| \leq 2\|f\|_{X} \frac{\left|z-z_{0}\right|}{\left|z-z_{0}\right|} \\
& \leq 2 \sup _{z \neq z_{0}} \frac{1}{\left|z-z_{0}\right|}\left|z-z_{0}\right|\|f\|_{X} \\
& \leq C\left|z-z_{0}\right|\left(\|f\|_{X}+\left\|f^{\prime}\right\|_{X}\right), \forall f \in D^{1}(X) .
\end{aligned}
$$

Em suma, para todo $z \in X$,

$$
\left|f(z)-f\left(z_{0}\right)\right| \leq C\left|z-z_{0}\right|\left(\|f\|_{X}+\left\|f^{\prime}\right\|_{X}\right), \forall f \in D^{1}(X) .
$$


A partir do Teorema 3.2.5, temos que se $X$ se escreve como união finita de conjuntos uniformemente regulares, então $D(X, M)$ é Banach. Em particular, cada conjunto uniformemente regular é conexo por caminhos em $\mathbb{C}$, logo é conexo. Desse modo, um conjunto que se escreve como união finita de conjuntos uniformemente regulares possui uma quantidade finita de componentes conexas.

A partir disso, Bland e Feinstein mostraram em [7], quase 30 anos depois, que se $X$ possuir uma quantidade infinita de componentes conexas, $D(X, M)$ não será uma álgebra de Banach. Antes de apresentarmos este resultado, demonstraremos um Lema auxiliar.

Lema 3.2.6. Se $X \subset \mathbb{C}$ possui quantidade infinita de componentes conexas, então existe sequência $\left(F_{n}\right)$ de subconjuntos em $X$ satisfazendo:

1. $F_{n}$ é não vazio, aberto e fechado de $X, \forall n \in \mathbb{N}$.

2. $F_{n} \cap F_{m}=\emptyset, \forall n \neq m$.

Demonstração. Uma vez que $X$ possui quantidade infinita de componentes conexas, $X$ não pode ser conexo. Logo, existem $E_{1}, F_{1} \subset E_{0}$ não-vazios, disjuntos, abertos e fechados de $X$ tais que $E_{0}=E_{1} \cup F_{1}$. Sem perda de generalidade, podemos supor que $E_{1}$ possui quantidade infinita de componentes conexas. Analogamente, temos que $E_{1}$ não é conexo, então, existem $E_{2}, F_{2} \subset E_{1}$ não-vazios, disjuntos, abertos e fechados de $E_{1}$ tais que $E_{1}=E_{2} \cup F_{2}$. Sem perda de generalidade, suponha que $E_{2}$ possua quantidade infinita de componentes conexas. Além disso, em particular, $E_{2}$ e $F_{2}$ são abertos e fechados de $X$, e $F_{1} \cap F_{2}=\emptyset$.

Indutivamente, suponha que existam $E_{1}, \cdots, E_{n}$ e $F_{1}, \cdots, F_{n}$ não-vazios, disjuntos, abertos e fechados de $X$ tais que $E_{i}=E_{i+1} \cup F_{i+1}$ de modo que cada $E_{i}$ possua quantidade infinita de componentes conexas. Mas como $E_{n}$ não pode ser conexo, existem $E_{n+1}, F_{n+1} \subset E_{n}$ não-vazios, disjuntos, abertos e fechados de $E_{n}$ tais que $E_{n}=E_{n+1} \cup F_{n+1}$. Sem perda de generalidade, admita que $E_{n+1}$ possui quantidade enumerável de componentes conexas.

Em suma, mostramos que existe sequência $\left(F_{n}\right)$ de subconjuntos de $X$ satisfazendo a condição 1. Para verificarmos a condição 2, considere $n \neq m$ naturais. Sem perda generalidade, suponha $n<m$. Assim, $F_{m} \subset E_{m-1} \subset E_{n}$ e $E_{n} \cap F_{n}=\emptyset$. Concluímos que $F_{n} \cap F_{m}=\emptyset, \forall n \neq m$.

Teorema 3.2.7. (Bland - Feinstein, [7])

Se $X$ possuir quantidade infinita de componentes conexas, então $D(X, M)$ não é álgebra de Banach.

Demonstração. Segue do Lema 3.2.6 que existe uma sequência $\left(F_{n}\right)$ de subconjuntos não vazios de $X$ que são abertos e fechados em $X$ e $F_{n} \cap F_{m}=\emptyset, \forall n \neq m$. Assim, dado $n>0$, existe $z_{n} \in F_{n}$, pois $F_{n}$ é não-vazio. Desse modo, $\left(z_{n}\right)$ é uma sequência em $X$ e, por compacidade, existe subsequência $z_{n_{k}} \rightarrow \bar{z} \in X$. Além disso, como $F_{n_{k}}$ é aberto de $X, \bar{z} \notin F_{n_{k}}, \forall k$, pois $F_{n} \cap F_{m}=\emptyset, \forall n \neq m$. Seja $f: X \rightarrow \mathbb{C}$ definida da seguinte maneira:

$$
f(z)= \begin{cases}z_{n_{k}} & \text { se } z \in F_{n_{k}} \\ \bar{z} & \text { se } z \in X \backslash A\end{cases}
$$

onde $A=\bigcup_{k=1}^{\infty} F_{n_{k}}$.

Vamos mostrar que $f \in C(X)$. De fato, dados $k \geq 1$ e $z_{0} \in F_{n_{k}}$, como $F_{n_{k}}$ é aberto de $X$, existe $V \subset F_{n_{k}}$ vizinhança aberta de $z_{0}$. Logo, $\left.f\right|_{V}=f\left(z_{0}\right)$ que é constante e por conseguinte $f$ é contínua em $z_{0}$. Como $z_{0}$ é um ponto arbitrário em $F_{n_{k}}$, segue que $f$ é contínua em $F_{n_{k}}$, para todo $k \geq 1$.

Dado $\epsilon>0$, existe $k_{0} \in \mathbb{N}$ tal que $\left|z_{n_{k}}-\bar{z}\right|<\epsilon, \forall k \geq k_{0}$. Seja $U=B(\bar{z}, \epsilon) \cap\left(\bigcup_{k=1}^{k_{0}} X \backslash F_{n_{k}}\right)$. Como cada $F_{n_{k}}$ é fechado de $X, X \backslash F_{n_{k}}$ é aberto. Assim, $U$ é aberto de $X$ que contém $\bar{z}$. Logo, existe $\delta>0$ tal que $B(\bar{z}, \delta) \subset U$. Daí, para todo $z \in B(\bar{z}, \delta)$,

$$
|f(z)-f(\bar{z})| \leq \sup _{k \geq k_{0}}\left|z_{n_{k}}-\bar{z}\right|<\epsilon .
$$


Portanto, $f$ é contínua em $\bar{z}$.

Dado $z_{0} \in(X \backslash A)^{\circ}$, existe $V \subset X \backslash A$ vizinhança aberta de $z_{0}$ tal que $\left.f\right|_{V}$ é constante. Logo, $f$ é contínua no interior de $X \backslash A$.

Como $X \backslash A$ é fechado, resta verificar a continuidade de $f$ em $\partial(X \backslash A)$. Com efeito, sejam $z_{0} \in$ $\partial(X \backslash A)$ e $\left(x_{n}\right)$ sequência em $X$ convergindo para $z_{0}$. Passando para subsequências se necessário, suponha, sem perda de generalidade, que $\left(x_{n}\right) \subset A$ ou $\left(x_{n}\right) \subset X \backslash A$. Se $\left(x_{n}\right) \subset X \backslash A, f\left(x_{n}\right)=$ $\bar{z} \rightarrow \bar{z}=f\left(z_{0}\right)$.

Caso $\left(x_{n}\right) \subset A$, defina $A_{m}=\bigcup_{k \leq m} F_{n_{k}}$. Suponha, por contradição, que exista $m \in \mathbb{N}$ tal que $\left(x_{n}\right)$ possui subsequência contida em $A_{m}$. Como $A_{m}$ é fechado de $X$, pois é união finita de conjuntos fechados de $X$, e $x_{n} \rightarrow z_{0}$, segue que $z_{0} \in A_{m}$. Uma vez que $X \backslash A$ é fechado, temos

$$
z_{0} \in \partial(X \backslash A) \subset X \backslash A \Rightarrow z \in A_{m} \cap(X \backslash A) \subset A \cap(X \backslash A)=\emptyset,
$$

uma contradição. Então, dado $m \in \mathbb{N}$, não existe subsequência de $\left(x_{n}\right)$ contida em $A_{m}$, i.e existe $N_{m} \in \mathbb{N}$ tal que $x_{n} \in A \backslash A_{m}, \forall n \geq N_{m}$. Assim, dado $\epsilon>0$, existe $k_{0} \in \mathbb{N}$ tal que $\left|z_{n_{k}}-\bar{z}\right|<\epsilon, \forall k \geq$ $k_{0}$. Por outro lado, existe $N_{k_{0}} \in \mathbb{N}$ tal que $x_{n} \in A \backslash A_{k_{0}}, \forall n \geq N_{k_{0}}$. Daí, $f\left(x_{n}\right) \in\left\{z_{n_{k}}: k>k_{0}\right\}$. Logo,

$$
\left|f\left(x_{n}\right)-f\left(z_{0}\right)\right| \leq \sup _{k>k_{0}}\left|z_{n_{k}}-z_{0}\right|<\epsilon \Rightarrow f\left(x_{n}\right) \rightarrow f\left(z_{0}\right) .
$$

Portanto, $f$ é contínua em $z_{0}$. Em suma, provamos que $f$ é contínua em todo ponto de $X$.

Vamos provar agora que $f \notin D^{1}(X)$. De fato, como $f$ é constante em cada aberto e fechado $F_{n_{k}}$, sua derivada é igual a $0 \mathrm{em} A$. Desse modo, se $f \in D^{1}(X)$, como $z_{n_{k}} \rightarrow \bar{z}, f^{\prime}\left(z_{0}\right)=\lim f^{\prime}\left(z_{n_{k}}\right)=0$. Não obstante, $\frac{f\left(z_{n_{k}}\right)-f(\bar{z})}{z_{n_{k}}-\bar{z}}=1$. Logo $f \notin D^{1}(X)$.

Em seguida, vamos construir uma sequência de Cauchy $\left(f_{i}\right)$ em $D(X, M)$ que converge uniformemente para $f$ em $X$. Para cada $i \in \mathbb{N}$, defina

$$
f_{i}(z)=\left\{\begin{array}{ll}
z_{n_{k}} & \text { se } z \in F_{n_{k}} \text { e } k \leq i \\
\bar{z} & \text { se } z \in X \backslash \bigcup_{k=1}^{i} F_{n_{k}}
\end{array} .\right.
$$

Como $F_{n_{k}}$ é aberto e fechado de $X$, para cada $i \in \mathbb{N}$, temos $\left.f_{i}^{\prime}\right|_{F_{n_{k}}}=0, \forall k \leq i$. Além disso, $X \backslash$ $\cup_{k=1}^{i} F_{n_{k}}$ também é aberto de $X, \operatorname{logo} f_{i}^{\prime}(z)=0$ em $X \backslash \cup_{k=1}^{i} F_{n_{k}}$. Então, $f_{i}^{\prime}=0$. Daí, $f_{i}^{(k)}=0, \forall k \geq$ 1. Logo, $f_{i} \in D(X, M), \forall i \in \mathbb{N}$. Em particular, também temos que $\left\|f_{i}-f_{j}\right\|=\left\|f_{i}-f_{j}\right\|_{X}, \forall i, j \in \mathbb{N}$. Daí,

$$
\left\|f_{i}-f_{j}\right\|=\sup _{z \in X}\left|f_{i}(z)-f_{j}(z)\right|=\left\{\begin{array}{c}
0, \quad \text { se } z \in F_{n_{k}}, k \leq i \text { ou } z \in X \backslash \cup_{k=1}^{j} F_{n_{k}} \\
\left|\bar{z}-z_{n_{k}}\right|, \quad \text { se } z \in F_{n_{k}}, \text { onde } k=i+1, \cdots, j
\end{array} .\right.
$$

Desse modo, dado $\epsilon>0$, existe $k_{0} \in \mathbb{N}$ tal que $\left|\bar{z}-z_{n_{k}}\right|<\epsilon, \forall k \geq k_{0}$. Isto é, para todos $i, j \geq k_{0}$, temos $k \geq k_{0}$ e, portanto,

$$
\left\|f_{i}-f_{j}\right\|=\sup _{k=i+1, \cdots, j}\left|\bar{z}-z_{n_{k}}\right|<\epsilon .
$$

Para concluir, vamos mostrar que $\left(f_{i}\right)$ converge para $f$ em $C(X)$. Como

$$
\left\|f_{i}-f\right\|_{X}=\sup _{z \in X}\left|f_{i}(z)-f(z)\right|=\left\{\begin{array}{cc}
0, & \text { se } z \in F_{n_{k}}, k \leq i \text { ou } z \in X \backslash \cup_{k=1}^{i} F_{n_{k}} \\
\left|z_{n_{k}}-\bar{z}\right|, & \text { se } z \in F_{n_{k}}, \text { onde } k \geq i+1
\end{array}\right.
$$

temos $\left\|f_{i}-f\right\|_{X}=\sup _{z \in X}\left|f_{i}(z)-f(z)\right|=\sup _{k \geq i+1}\left|z_{n_{k}}-\bar{z}\right| \rightarrow 0$ quando $i \rightarrow \infty$, pois $z_{n_{k}} \rightarrow \bar{z}$.

Em suma, $\left(f_{i}\right)$ é uma sequência de Cauchy em $D(X, M)$ que converge uniformemente para $f \in C(X)$, entretanto $f \notin D(X, M)$. Conclusão: $D(X, M)$ não é álgebra de Banach.

A seguir apresentaremos um exemplo de um subconjunto de $\mathbb{C}$ que é compacto, perfeito e possui quantidade infinita de componentes conexas. 
Exemplo 3.2.8. O conjunto

$$
X=\left\{x+i y \in \mathbb{C}:\left(x=0 \text { ou } \frac{1}{x} \in \mathbb{N}\right) \text { e } y \in[0,1]\right\}
$$

é compacto, perfeito e possui quantidade infinita de componentes conexas.

De fato, não é difícil ver que $X$ é compacto e perfeito. Além disso, temos que

$$
X=\{0\} \times[0,1] \cup\left(\bigcup_{n \in \mathbb{N}}\{1 / n\} \times[0,1]\right),
$$

i.e $X$ é uma união enumerável disjunta de conexos.

\section{$3.3 \quad$ O Espectro de $D(X, M)$}

Antes de estudar o espectro de $D(X, M)$, veremos alguns resultados importantes provados por Dales e Davie em [11]. Nesta seção, $M=\left(M_{n}\right)$ denotará uma sequência de álgebra. Além disso, vamos considerar a seguinte notação para uma sequência de álgebra $M$ :

$$
d(M)=\lim _{n \rightarrow \infty}\left(\frac{n !}{M_{n}}\right)^{1 / n} .
$$

Primeiramente, iniciamos a seção com um resultado envolvendo funções de Whitney que podem ser encontrados no livro de Malgrange [28].

Sejam $X \subset \mathbb{C}$ compacto e $f \in D^{1}(X)$. Dizemos que $f$ é uma função de Whitney em $X$ se $\forall \epsilon>0, \exists \delta>0,\left|f(y)-f(x)-f^{\prime}(x)(y-x)\right| \leq|x-y| \epsilon$, para todos $x, y \in X$ tais que $|x-y|<\delta$.

O próximo resultado é chamado de Teorema da Extensão de Whitney cuja demonstração pode ser encontrada no capítulo 1 do livro [28].

Lema 3.3.1. (Teorema da Extensão de Whitney) Sejam $X \subset \mathbb{C}$ compacto e $f: X \rightarrow \mathbb{C}$ uma função de Whitney em $X$. Então, existem um aberto $\Omega \subset \mathbb{C}$ que contém $X$ e uma função $F: \Omega \rightarrow \mathbb{C}$ com derivada contínua tal que

$$
\left.F\right|_{X}=f,\left.\quad \frac{\partial F}{\partial z}\right|_{X}=f^{\prime},\left.\quad \frac{\partial F}{\partial \bar{z}}\right|_{X}=0
$$

Dales e Davie usaram o Teorema 2.2.8 e os Lemas 3.2.4 e 3.3.1 para demonstrar o seguinte Teorema:

Teorema 3.3.2. (Dales-Davie, [11]) Se $X$ é uniformemente regular, então $D^{1}(X) \subset R(X)$.

Demonstração. Como $X$ é uniformemente regular, existe $C>0, \delta(z, w) \leq C|z-w|, \forall z, w \in X$. Sejam $f \in D^{1}(X)$ e $g_{x}(y)=f(y)-f(x)-f^{\prime}(x)(y-x), x, y \in X$.

Dados $x, y \in X$, seja $\alpha:[0,1] \rightarrow X$ curva que liga $x$ e $y$ de comprimento $\delta(x, y)$ e considere $Y=\alpha([0,1])$. Pelo Lema 1.2.16, temos que $Y$ é uniformemente regular. Desse modo, pelo Lema 3.2.4:

$$
\left|g_{x}(y)-g_{x}(x)\right| \leq \delta(x, y)\left\|g_{x}^{\prime}\right\|_{Y} \leq C|x-y| \sup _{z \in Y}\left|f^{\prime}(z)-f^{\prime}(x)\right| .
$$

Como $g_{x}(x)=0$, segue que

$$
\left|g_{x}(y)\right| \leq C|x-y| \sup _{z \in Y}\left|f^{\prime}(z)-f^{\prime}(x)\right| .
$$


Daí, pela continuidade uniforme de $f^{\prime}$, dado $\epsilon>0, \exists \delta^{\prime}>0$ tal que se $|z-w|<\delta^{\prime}$, temos $\left|f^{\prime}(z)-f^{\prime}(w)\right|<\epsilon / C$. Tomando $\delta=\delta^{\prime} / C$, se $|x-y|<\delta$, obtemos

$$
|x-z| \leq \delta(x, z) \leq \delta(x, y) \leq C|x-y|, \forall z \in Y .
$$

Assim, $\sup _{z \in Y}\left|f^{\prime}(z)-f^{\prime}(x)\right|<\epsilon / C$. Como consequência obtemos

$$
\left|g_{x}(y)\right|<\epsilon|x-y| \text { quando }|x-y|<\delta .
$$

Desse modo, segue que $f$ é uma função de Whitney em $X$. Pelo Lema 3.3.1, existem $\Omega \subset \mathbb{C}$ um aberto contendo $X$ e $F: \Omega \rightarrow \mathbb{C}$ uma função com derivada contínua tal que $\left.F\right|_{X}=f$ e $\left.\frac{\partial F}{\partial \bar{z}}\right|_{X}=0$. Assim, aplicando o Teorema 2.2.8, concluímos que $f \in R(X)$.

O próximo Teorema, provado por Dales e Davie em 1973, apresenta uma condição necessária e suficiente para a álgebra $R_{0}(X)$ esteja contida em $D(X, M)$.

Teorema 3.3.3. (Dales -Davie, [11]) Sejam $X \subset \mathbb{C}$ compacto e perfeito. Então,

$$
R_{0}(X) \subset D(X, M) \Longleftrightarrow d(M)=\lim _{n \rightarrow \infty}\left(\frac{n !}{M_{n}}\right)^{1 / n}=0 .
$$

Demonstração. Suponha que $d(M) \neq 0$, i.e a sequência $\left(\left(\frac{M_{n}}{n !}\right)^{1 / n}\right)_{n \in \mathbb{N}_{0}}$ é limitada. Seja $C>0$ tal que $\left(\frac{M_{n}}{n !}\right)^{1 / n} \leq C, \forall n \in \mathbb{N}_{0}$. Dado $f_{\alpha}(z)=\frac{1}{\alpha-z}, z \in X, \alpha \notin X$, temos $f_{\alpha}^{(k)}(z)=\frac{(-1)^{k} k !}{(\alpha-z)^{k+1}}, \forall k \geq 1$. Vamos provar que para algum $\alpha, f_{\alpha} \notin D(X, M)$, isto é $\sum_{k=0}^{\infty}\left\|f_{\alpha}^{(k)}\right\|_{X} / M_{n}=+\infty$. Com efeito, seja

$$
a_{k}=\frac{1}{M_{k}}\left\|f_{\alpha}^{(k)}\right\|_{X}=\frac{k !}{M_{k}} \sup _{z \in X}\left|\frac{1}{\alpha-z}\right|^{k+1}, \forall k \in \mathbb{N}_{0} .
$$

Para cada $k \in \mathbb{N}_{0}$, temos que a função $\left(\alpha \in \mathbb{C} \backslash X \mapsto\left(\sup _{z \in X}\left|\frac{1}{\alpha-z}\right|^{k+1}\right)^{1 / k}\right)$ tende para $+\infty$ quando $d(\alpha, X) \rightarrow 0$. Então, existe $\delta_{k}>0$ tal que

$$
\left(\sup _{z \in X}\left|\frac{1}{\alpha-z}\right|^{k+1}\right)^{1 / k}>2 C, \text { quando } \alpha \in \mathbb{C} \backslash X \text { e } d(\alpha, X) \leq \delta_{k} .
$$

Como $\mathbb{C} \backslash X$ é um aberto, se $\delta=\min \left\{\delta_{k}: k \in \mathbb{N}_{0}\right\}$, existe $\alpha \in \mathbb{C} \backslash X$ tal que $d(\alpha, X) \leq \delta$. Portanto,

$$
\left(a_{k}\right)^{1 / k}=\left(\frac{k !}{M_{k}}\right)^{1 / k}\left(\sup _{z \in X}\left|\frac{1}{\alpha-z}\right|^{k+1}\right)^{1 / k}>\frac{1}{C} 2 C=2>1 .
$$

Assim, limsup $\left(a_{k}\right)^{1 / k}>1$. Pelo Teste da Raiz, temos $\sum a_{k}=+\infty$. Donde, $f_{\alpha} \notin D(X, M)$.

Reciprocamente, suponha $d(M)=0$. Como $D(X, M)$ é uma álgebra que contém os polinômios, é suficiente provar que $1 / q \in D(X, M), \forall q \in \mathbb{C}[z], q(x) \neq 0, \forall x \in X$. Desse modo, é suficiente verificar que $\frac{1}{\alpha-z} \in D(X, M), \forall \alpha \notin X$. Com efeito, dado $\alpha \notin X$, seja $f_{\alpha}(z)=\frac{1}{\alpha-z}, z \in X$. Como $f_{\alpha}$ é contínua no compacto $X, f_{\alpha}$ é limitada, logo existe $C>0$ tal que $\sup _{z \in X}\left|\frac{1}{\alpha-z}\right| \leq C$. Daí,

$$
\left(a_{k}\right)^{1 / k}=\left(\frac{1}{M_{k}}\left\|f_{\alpha}^{(k)}\right\|_{X}\right)^{1 / k}=\left(\frac{k !}{M_{k}}\right)^{1 / k}\left(\sup _{z \in X}\left|\frac{1}{\alpha-z}\right|^{k+1}\right)^{1 / k} \leq\left(\frac{k !}{M_{k}}\right)^{1 / k} C^{1+1 / k}
$$


Como $d(M)=0$ e $\lim _{k \rightarrow \infty} C^{1 / k}=1$, segue que $\lim _{k \rightarrow \infty}\left(a_{k}\right)^{1 / k}=0<1$. Pelo Teste da Raiz, segue que $\sum a_{k}<\infty$. Portanto, $f_{\alpha} \in D(X, M)$.

A partir dos Teoremas 3.3.2 e 3.3.3, temos o seguinte corolário:

Corolário 3.3.4. Se $X$ é uniformemente regular e $d(M)=0$, então $\overline{D(X, M)}=R(X)$.

Demonstração. Pelos Teoremas 3.3.2 e 3.3.3, segue que $R_{0}(X) \subset D(X, M) \subset D^{1}(X) \subset R(X)$. Desse modo, concluímos que

$$
R(X)=\overline{R_{0}(X)} \subset \overline{D(X, M)} \subset R(X) .
$$

Agora, podemos apresentar um resultado de Dales e Davie que mostra condições para que $D(X, M)$ seja uma álgebra natural.

Proposição 3.3.5. (Dales-Davie, [11]) Se $X$ é um conjunto uniformemente regular, $d(M)=0 e$ $\|f\|_{X}=\|\hat{f}\|_{\mathcal{M}_{D(X, M)}}, \forall f \in D(X, M)$, então $D(X, M)$ é natural.

Demonstração. Pelo Teorema 2.2.7, segue que $\mathcal{M}_{\overline{D(X, M)}} \cong \mathcal{M}_{D(X, M)}$. Por outro lado, a Proposição 2.3.11 implica que $X \cong \mathcal{M}_{R(X)}$. Mas como $\overline{D(X, M)}=R(X)$ (Corolário 3.3.4), concluímos que $X \cong \mathcal{M}_{D(X, M)}$.

Denotaremos os fechos de $\mathbb{C}[z]$ e $R_{0}(X)$ em $D(X, M)$ por $D_{P}(X, M)$ e $D_{R}(X, M)$, respectivamente. Já vimos que se $D^{1}(X)$ é uma álgebra de Banach, $D(X, M)$ também o é. Neste caso, $D_{P}(X, M)$ e $D_{R}(X, M)$ também são álgebras de Banach, pois são fechadas em $D(X, M)$. Os Teoremas 3.3.6 e 3.3.7 apresentam resultados a respeito espectros dessas álgebras. O próximo resultado utiliza uma técnica de demonstração análoga à 2.3.11.

Teorema 3.3.6. (Dales-Davie, [11]) Se X é uniformemente regular e se $M=\left(M_{n}\right)$ é uma sequência de álgebra tal que $d(M)=0$, então $D_{R}=D_{R}(X, M)$ é natural.

Demonstração. Como $X$ é uniformemente regular, $D_{R}$ é Banach. Por outro lado, como $d(M)=0$, temos, como consequência do Teorema 3.3.3, $R_{0}(X) \subset D_{R}$.

Sejam $\varphi \in \mathcal{M}_{D_{R}}$ e $z_{0}=\varphi(i d)$, onde $i d(z)=z, \forall z \in \mathbb{C}$. Vamos provar que $\varphi=\varphi_{z_{0}}$. Analogamente à Proposição 2.3.11, temos que $\varphi(r)=r\left(z_{0}\right), \forall r \in R_{0}(X)$, e $z_{0} \in X$. Além disso, dado $f \in D_{R}$, existe sequência $\left(r_{n}\right)$ em $R_{0}(X)$ que converge para $f$ em $D(X, M)$. A continuidade de $\varphi$ implica em $r_{n}\left(z_{0}\right)=\varphi\left(r_{n}\right) \rightarrow \varphi(f)$ em $\mathbb{C}$.

Mas, por outro lado, como $\left\|r_{n}-f\right\| \rightarrow 0$, temos $\left\|r_{n}-f\right\|_{X} \rightarrow 0$. Logo, $r_{n}\left(z_{0}\right) \rightarrow f\left(z_{0}\right)$. Assim, pela unicidade do limite, $\varphi(f)=f\left(z_{0}\right), \forall f \in D_{R}$.

O próximo teorema foi provado primeiramente por Dales e Davie em [11]. Quase 20 anos depois, Honary [18] provou, de forma mais simples, o mesmo resultado utilizando 2.3.5, como veremos a seguir.

Teorema 3.3.7. (Dales - Davie, [11] / Honary - [18]) Sejam $X \subset \mathbb{C}$ uniformemente regular $e$ $M=\left(M_{n}\right)$ uma sequência de álgebra tal que $d(M)=0$. Então, o espectro de $D_{P}=D_{P}(X, M)$ é homeomorfo a $\hat{X}$.

Demonstração. Uma vez que $X$ é uniformemente regular, $D_{P}$ e $D_{R}$ são álgebras de Banach tais que $D_{P} \subset D_{R}$. Como, pelo Teorema 3.3.6, $D_{R}$ é natural, segue da Proposição 2.3.5 que $\mathcal{M}_{\overline{D_{P}}} \cong \mathcal{M}_{D_{P}}$. Mas como $\mathbb{C}[z] \subset D_{P} \subset P(X)$, temos $\overline{D_{P}}=P(X)$. Daí, $\mathcal{M}_{D_{P}} \cong \mathcal{M}_{P(X)} \cong \hat{X}$.

Em 3.3.5, 3.3.6 e 3.3.7 uma das hipóteses era que $d(M)=0$. A seguir, mostraremos uma classe de sequências de álgebras que satisfazem esta condição. 
Exemplo 3.3.8. Seja $M_{0}=1$ e $M_{n}=n !^{\alpha}$, onde $\alpha>1$. Daí, $M=\left(M_{n}\right)$ é uma sequência de álgebra tal que

$$
\left(\frac{n !}{M_{n}}\right)^{1 / n}=\left(\frac{1}{n !^{\alpha-1}}\right)^{1 / n}=\frac{1}{\left(n !^{1 / n}\right)^{\alpha-1}} \rightarrow 0, \text { pois } \lim n !^{1 / n}=\infty, \text { implicando } d(M)=0 .
$$

Devido a dificuldade para apresentar exemplos de álgebras de Dales-Davie que satisfazem a condição $\|f\|_{X}=\|\hat{f}\|_{\mathcal{M}_{D}}$, Abtahi e Honary foram buscar condições sobre $M=\left(M_{n}\right)$ de modo que a igualdade seja válida. No que segue deste capítulo, apresentaremos os resultados do texto [2].

O próximo resultado foi demonstrado por Abtahi e Honary em [2]. Ele será importante para demonstrar o Teorema 3.3.12.

Lema 3.3.9. (Abtahi - Honary, [2]) Sejam $K>0$ e $\left(\epsilon_{m}\right)$ sequência em $\mathbb{R}_{+}$tal que $\epsilon_{m} \rightarrow 0$ quando $m \rightarrow \infty$. Então,

$$
\limsup _{p \rightarrow \infty}\left(\sum_{p=0}^{\infty}\left(\begin{array}{c}
p \\
m
\end{array}\right) \epsilon_{m}^{m} K^{p-m}\right)^{1 / p} \leq K .
$$

Demonstração. Dado $\epsilon>0$, existe $N_{0} \in \mathbb{N}, \epsilon_{m}<\epsilon K, \forall m \geq N_{0}$. Em particular, se $p>N_{0}$ e $m=0,1, \cdots, N_{0}$, temos

$$
\left(\begin{array}{c}
p \\
m
\end{array}\right)=\frac{p !}{m !(p-m) !} \leq \frac{p !}{(p-m) !}=p(p-1) \cdots(p-m+1) \leq p^{N_{0}} .
$$

Assim,

$$
\begin{aligned}
\sum_{m=0}^{p}\left(\begin{array}{c}
p \\
m
\end{array}\right) \epsilon_{m}^{m} K^{p-m} & =\sum_{m=0}^{N_{0}}\left(\begin{array}{c}
p \\
m
\end{array}\right) \epsilon_{m}^{m} K^{p-m}+\sum_{m=N_{0}+1}^{p}\left(\begin{array}{c}
p \\
m
\end{array}\right) \epsilon_{m}^{m} K^{p-m} \\
& <p^{N_{0}} \sum_{m=0}^{N_{0}} \epsilon_{m}^{m} K^{p-m}+\sum_{m=N_{0}+1}^{p}\left(\begin{array}{c}
p \\
m
\end{array}\right) \epsilon^{m} K^{p} \\
& <p^{N_{0}} \sum_{m=0}^{N_{0}} \epsilon_{m}^{m} K^{p-m}+K^{p} \sum_{m=0}^{p}\left(\begin{array}{c}
p \\
m
\end{array}\right) \epsilon^{m} \\
& <p^{N_{0}} K^{p} \sum_{m=0}^{N_{0}} \epsilon_{m}^{m} K^{-m}+K^{p}(1+\epsilon)^{p} \\
& <2 p^{N_{0}} K^{p}(1+\epsilon)^{p}\left(\sum_{m=0}^{N_{0}} \epsilon_{m}^{m} K^{-m}\right)\left(\frac{1}{2(1+\epsilon)^{p}}+\frac{1}{2 p^{N_{0}} \sum_{m=0}^{N_{0}} \epsilon_{m}^{m} K^{-m}}\right)
\end{aligned}
$$

Temos que $\frac{1}{2(1+\epsilon)^{p}} \leq \frac{1}{2}$ para todo $p$. Além disso, existe $p_{0} \in \mathbb{N}$ tal que $\frac{1}{p_{0}^{N_{0}}} \leq \sum_{m=0}^{N_{0}} \epsilon_{m}^{m} K^{-m}$. Assim,

$$
\frac{1}{2 p^{N_{0}} \sum_{m=0}^{N_{0}} \epsilon_{m}^{m} K^{-m}} \leq \frac{1}{2}, \forall p>p_{0} .
$$

Daí, se $p>\max \left\{N_{0}, p_{0}\right\}$, segue que

$$
\sum_{m=0}^{p}\left(\begin{array}{c}
p \\
m
\end{array}\right) \epsilon_{m}^{m} K^{p-m} \leq 2 p^{N_{0}} K^{p}(1+\epsilon)^{p}\left(\sum_{m=0}^{N_{0}} \epsilon_{m}^{m} K^{-m}\right) .
$$


Mas como, $\lim _{p \rightarrow \infty}\left(2 p^{N_{0}} \sum_{m=0}^{N_{0}} \epsilon_{m}^{m} K^{-m}\right)^{1 / p}=0$, concluímos que

$$
\limsup _{p \rightarrow \infty}\left(\sum_{p=0}^{\infty}\left(\begin{array}{c}
p \\
m
\end{array}\right) \epsilon_{m}^{m} K^{p-m}\right)^{1 / p} \leq K(1+\epsilon), \quad \forall \epsilon>0 .
$$

Como $\epsilon>0$ é arbitrário, podemos tomar $\epsilon \rightarrow 0$ e obtemos:

$$
\limsup _{p \rightarrow \infty}\left(\sum_{p=0}^{\infty}\left(\begin{array}{c}
p \\
m
\end{array}\right) \epsilon_{m}^{m} K^{p-m}\right)^{1 / p} \leq K .
$$

Em 1973, Dales e Davie definiram uma sequência $\left(P_{n}\right)$ para uma dada sequência de álgebra. Mais precisamente, dada uma sequência de álgebra $\left(M_{n}\right)$, vamos considerar $P_{n}=M_{n} / n$ ! para todo $n \geq 0$. A seguir, mostraremos algumas propriedades dessas sequências que serão necessárias para o decorrer desta dissertação.

Lema 3.3.10. Sejam $M=\left(M_{n}\right)$ uma sequência de álgebra e $P_{n}=M_{n} / n$ !, para todo $n \geq 0$. Então,

1. $P_{i} P_{j} \leq P_{i+j}, \forall i, j \geq 0$.

2. $\left(P_{i}\right)^{n} \leq P_{n i}$, para inteiros positivos $i$ e $n$.

3. Dados inteiros positivos $a_{1}, \cdots, a_{n}$ tais que $\sum_{k=1}^{n} k a_{k}=n$, temos

$$
\prod_{k=1}^{n}\left(P_{k}\right)^{a_{k}} \leq P_{n}
$$

Demonstração. 1. Dados $i, j \geq 0$, considere $n=i+j$ e $i=k$, daí

$$
\frac{P_{i} P_{j}}{P_{i+j}}=\frac{M_{i} M_{j}}{M_{i+j}} \cdot \frac{(i+j) !}{i ! j !}=\frac{M_{k} M_{n-k}}{M_{n}} \cdot \frac{n !}{k !(n-k) !} \leq 1 .
$$

2. Se $i=j$, do item 1 , temos $P_{i}^{2} \leq P_{2 i}$. Dado $n$, suponha que $P_{i}^{n} \leq P_{n i}$. Então,

$$
P_{i}^{n+1}=P_{i}^{n} P_{i} \leq P_{n i} P_{i} \leq P_{(n+1) i} .
$$

3. Dados inteiros positivos $a_{1}, \cdots, a_{n}$ tais que $\sum_{k=1}^{n} k a_{k}=n$, temos

$$
\prod_{k=1}^{n}\left(P_{k}\right)^{a_{k}} \leq \prod_{k=1}^{n} P_{k a_{k}} \leq P_{\sum_{k=1}^{n} k a_{k}} \leq P_{n}
$$

Tendo em vista o item 3 do Lema 3.3.10 e o conjunto $S(m, n)$ definido em 1.1, dados $n \geq m$ e $\left(a_{1}, \cdots, a_{n}\right) \in S(n, m)$, segue que

$$
\frac{1}{P_{n}} \prod_{k=1}^{n}\left(P_{k}\right)^{a_{k}} \leq 1
$$

Desse modo, podemos definir:

$$
A_{m}=\sup \left\{\frac{1}{P_{n}} \prod_{k=1}^{n}\left(P_{k}\right)^{a_{k}}: n \geq m, \quad\left(a_{1}, \cdots, a_{n}\right) \in S(n, m)\right\} .
$$


Proposição 3.3.11. Com a notação acima, se $M=\left(M_{n}\right)$ é uma sequência de álgebra, temos que

$$
\lim _{m \rightarrow \infty} A_{m}^{1 / m}=0 \Rightarrow d(M)=0 .
$$

Demonstração. Dado $\epsilon>0$, existe $N_{0} \in \mathbb{N}$ tal que $A_{m}^{1 / m}<\epsilon P_{1}, \forall m \geq N_{0}$. Em particular, se $n=m \geq N_{0}$ e $a_{1}=n, a_{2}=\cdots=a_{n}=0$, temos

$$
\left(\frac{n !}{M_{n}}\right)^{1 / n}=\frac{1}{P_{n}^{1 / n}}=\left(\frac{1}{P_{n}} P_{1}^{n}\right)^{1 / n} \frac{1}{P_{1}} \leq A_{n}^{1 / n} / P_{1}<\epsilon, \text { o que implica que } d(M)=0
$$

Em [2], Abtahi e Honary mostraram que se $X$ é uniformemente regular e se a sequência $\left(A_{m}\right)$ definida em 3.6 é tal que $A_{m}^{1 / m} \rightarrow 0$, então $D(X, M)$ é uma álgebra natural.

Teorema 3.3.12. (Abtahi - Honary, [2]) Sejam $X$ uniformemente regular, $M=\left(M_{n}\right)$ sequência de álgebra e $\left(A_{m}\right)$ sequência definida em 3.6. Se $\lim _{m \rightarrow \infty} A_{m}^{1 / m}=0$, então $D=D(X, M)$ é natural.

Demonstração. A Proposição 3.3.11 implica em $d(M)=0$. Assim, pelo Teorema 3.3.5 e a Proposição 2.2.6, é suficiente provar que $\|\hat{f}\|_{\mathcal{M}_{D}} \leq\|f\|_{X}$, para toda função $f \in D$.

Com efeito, seja $F(y)=y^{p}, p \in \mathbb{N}$. Daí, como $F^{(m)}(y)=m !\left(\begin{array}{c}p \\ m\end{array}\right) y^{p-m}$, pela Fórmula de Faà di Bruno (1.1.1),

$$
\begin{aligned}
(F \circ f)^{(n)}(z) & =\sum_{m=0}^{n} F^{(m)}(f(z)) \sum_{\left(a_{1}, \cdots, a_{n}\right) \in S(m, n)}\left[\frac{n !}{a_{1} ! \cdots a_{n} !} \prod_{k=1}^{n}\left(\frac{f^{(k)}}{k !}\right)^{a_{k}}\right] \\
& =\sum_{m=0}^{\min \{p, n\}} m !\left(\begin{array}{c}
p \\
m
\end{array}\right) f^{p-m}(z) \sum_{\left(a_{1}, \cdots, a_{n}\right) \in S(m, n)}\left[\frac{n !}{a_{1} ! \cdots a_{n} !} \prod_{k=1}^{n}\left(\frac{f^{(k)}}{k !}\right)^{a_{k}}\right] .
\end{aligned}
$$

Logo,

$$
\begin{aligned}
\left\|f^{p}\right\| & =\|F \circ f\|=\sum_{n=0}^{\infty} \frac{1}{M_{n}}\left\|(F \circ f)^{(n)}\right\|_{X} \\
& \leq \sum_{n=0}^{\infty} \frac{1}{M_{n}} \sum_{m=0}^{\min \{p, n\}} m !\left(\begin{array}{c}
p \\
m
\end{array}\right)\|f\|_{X}^{p-m} \sum_{\left(a_{1}, \cdots, a_{n}\right) \in S(m, n)}\left[\frac{n !}{a_{1} ! \cdots a_{n} !} \prod_{k=1}^{n}\left(\frac{\left\|f^{(k)}\right\|_{X}}{k !}\right)^{a_{k}}\right] .
\end{aligned}
$$

Trocando a ordem da soma, temos

$$
\begin{aligned}
\left\|f^{p}\right\| & \leq \sum_{m=0}^{p}\left(\begin{array}{c}
p \\
m
\end{array}\right)\|f\|_{X}^{p-m} m ! \sum_{n=m}^{\infty} \sum_{\left(a_{1}, \cdots, a_{n}\right) \in S(m, n)}\left[\frac{1}{a_{1} ! \cdots a_{n} !} \frac{n !}{M_{n}} \prod_{k=1}^{n}\left(\frac{\left\|f^{(k)}\right\|_{X}}{k !}\right)^{a_{k}}\right] \\
& \leq \sum_{m=0}^{p}\left(\begin{array}{c}
p \\
m
\end{array}\right)\|f\|_{X}^{p-m} m ! \sum_{n=m}^{\infty} \sum_{\left(a_{1}, \cdots, a_{n}\right) \in S(m, n)}\left[\frac{1}{\prod_{k=1}^{n} a_{k} !} \frac{\prod_{k=1}^{n}\left(P_{k}\right)^{a_{k}}}{P_{n}} \prod_{k=1}^{n}\left(\frac{\left\|f^{(k)}\right\|_{X}}{P_{k} k !}\right)^{a_{k}}\right] \\
& \leq \sum_{m=0}^{p}\left(\begin{array}{c}
p \\
m
\end{array}\right)\|f\|_{X}^{p-m} A_{m} m ! \sum_{n=m}^{\infty} \sum_{\left(a_{1}, \cdots, a_{n}\right) \in S(m, n)}\left[\frac{1}{\prod_{k=1}^{n} a_{k} !} \prod_{k=1}^{n}\left(\frac{\left\|f^{(k)}\right\|_{X}}{M_{k}}\right)^{a_{k}}\right],
\end{aligned}
$$


pois $\frac{\prod_{k=1}^{n}\left(P_{k}\right)^{a_{k}}}{P_{n}} \leq A_{m}$ e $M_{k}=P_{k} k$ !. Segue do Corolário 1.1.4 para $B_{k}=\left\|f^{(k)}\right\|_{X} / M_{k}$ que

$$
\begin{aligned}
\left\|f^{p}\right\| & \leq \sum_{m=0}^{p}\left(\begin{array}{c}
p \\
m
\end{array}\right)\|f\|_{X}^{p-m} A_{m}\left(\sum_{k=1}^{\infty} \frac{\left\|f^{(k)}\right\|_{X}}{M_{k}}\right)^{m} \\
& \leq \sum_{m=0}^{p}\left(\begin{array}{c}
p \\
m
\end{array}\right)\|f\|_{X}^{p-m}\left(A_{m}^{1 / m}\left(\|f\|-\|f\|_{X}\right)\right)^{m} .
\end{aligned}
$$

Aplicando o Lema 3.3.9 para $\epsilon_{m}=A_{m}^{1 / m}\left(\|f\|-\|f\|_{X}\right)$ e $K=\|f\|_{X}$, segue que

$$
\limsup _{p \rightarrow \infty}\left\|f^{p}\right\|^{1 / p} \leq \limsup _{p \rightarrow \infty}\left[\sum_{m=0}^{p}\left(\begin{array}{c}
p \\
m
\end{array}\right)\|f\|_{X}^{p-m}\left(A_{m}^{1 / m}\left(\|f\|-\|f\|_{X}\right)\right)^{m}\right]^{1 / p} \leq\|f\|_{X} .
$$

Portanto,

$$
\|\hat{f}\|_{\mathcal{M}_{D}}=\limsup _{p \rightarrow \infty}\left\|f^{p}\right\|^{1 / p} \leq\|f\|_{X}
$$

A partir do Teorema 3.3.12, temos que se $X$ é uniformemente regular e $\left(A_{m}\right)^{1 / m} \rightarrow 0$, então $D(X, M)$ será uma álgebra natural. No que segue deste capítulo, apresentaremos condições que garantem $\left(A_{m}\right)^{1 / m} \rightarrow 0$. Nessa direção, começamos apresentado um resultado de Abtahi e Honary [2].

Proposição 3.3.13. (Abtahi - Honary, [2]) Seja $\left(P_{k}\right)$ sequência de números reais positivos tal que

$$
K=\sup _{i, j \in \mathbb{N}} \frac{P_{i} P_{j}}{P_{i+j-1}}<\infty .
$$

Então,

$$
\prod_{k=1}^{n}\left(P_{k}\right)^{a_{k}} \leq K^{m-1} P_{n-m+1}, \quad \forall m \in \mathbb{N}, \forall n \geq m, \forall\left(a_{1}, \cdots, a_{n}\right) \in S(n, m) .
$$

Reciprocamente, se $\left(P_{k}\right)$ é uma sequência que satisfaz a inequação 3.8 para alguma constante $K>0$, então

$$
\sup _{i, j \in \mathbb{N}} \frac{P_{i} P_{j}}{P_{i+j-1}} \leq K
$$

Demonstração. Suponha que $\left(P_{k}\right)$ satisfaça 3.7. Vamos provar a inequação 3.8 por indução em $m$.

Caso $m=1$. Para todo $n \geq 1$, se $\left(a_{1}, \cdots, a_{n}\right) \in S(1, n)$, temos $\sum_{k=1}^{n} a_{k}=1$ e $\sum_{k=1}^{n} k a_{k}=n$. Então, $a_{n}=1$ e $a_{1}=\cdots=a_{n-1}=0$. Daí

$$
\prod_{k=1}^{n}\left(P_{k}\right)^{a_{k}}=\left(P_{n}\right)^{a_{n}}=P_{n} \text {, implicando na inequação } 3.8 \text { para o caso em que } m=1 .
$$

Dado $m>1$, suponha a inequação 3.8 verdadeira para dados $n \geq m$ e $\left(a_{1}, \cdots, a_{n}\right) \in S(m, n)$. Sejam, pois, $n \geq m+1$ e $\left(a_{1}, \cdots, a_{n}\right) \in S(m+1, n)$. Então, $\sum_{k=1}^{n} a_{k}=m+1$ e $\sum_{k=1}^{n} k a_{k}=n$.

Em particular, se $a_{1}=\cdots=a_{n-1}=0$, teríamos $a_{n}=m+1 \Rightarrow n(m+1)=n$, o que implicaria $m=0$, contradição. Logo, existe $i \in\{1, \cdots, n-1\}$ tal que $a_{i} \geq 1$. Assim,

$$
\left\{\begin{array}{c}
a_{1}+\cdots+a_{i-1}+\left(a_{i}-1\right)+a_{i+1}+\cdots+a_{n}=m \\
a_{1}+\cdots+(i-1) a_{i-1}+i\left(a_{i}-1\right)+(i+1) a_{i+1}+\cdots+n a_{n}=n-i
\end{array}\right.
$$

Consequentemente, $n-i \geq m$. 
Além disso, se existisse algum $j>n-i$ tal que $a_{j}>0$, teríamos $n=\sum_{k=1}^{n} k a_{k} \geq i+j>$ $i+n-i=n$, uma contradição. Portanto, $a_{k}=0, \forall k>n-i$. Desse modo, se $b_{k}=a_{k}$, para $k=1, \cdots, i-1, i+1, \cdots, n-i$ e $b_{i}=a_{i}-1$, temos

$$
\sum_{k=1}^{n-i} b_{k}=m \text { e } \sum_{k=1}^{n-i} k b_{k}=n-i \text {. Donde, }\left(b_{1}, \cdots, b_{n-i}\right) \in S(n-i, m) .
$$

Pela hipótese de indução, segue que

$$
\prod_{k=1}^{n-i}\left(P_{k}\right)^{b_{k}}=\left(P_{1}\right)^{a_{1}} \cdots\left(P_{i-1}\right)^{a_{i-1}}\left(P_{i}\right)^{a_{i}-1}\left(P_{i+1}\right)^{a_{i+1}} \cdots\left(P_{n}\right)^{a_{n}} \leq K^{m-1} P_{n-i-m+1},
$$

isto é,

$$
\prod_{k=1}^{n}\left(P_{k}\right)^{a_{k}}\left(P_{i}\right)^{-1} \leq K^{m-1} P_{n-i-m+1}
$$

o que implica que

$$
\prod_{k=1}^{n}\left(P_{k}\right)^{a_{k}} \leq K^{m-1} P_{n-i-m+1} P_{i} .
$$

Mas, por hipótese, $P_{n-i-m+1} P_{i} \leq K P_{n-m}$. Logo,

$$
\prod_{k=1}^{n}\left(P_{k}\right)^{a_{k}} \leq K^{m} P_{n-m}=K^{(m+1)-1} P_{n-(m+1)-1}
$$

Reciprocamente, suponha que $\left(P_{k}\right)$ seja uma sequência que satisfaça a inequação 3.8 para algum constante $K>0$. Então, dados $i, j \in \mathbb{N}$, considere $m=2$ e $n=i+j$.

Caso $i \neq j$, considere $a_{i}=a_{j}=1$ e $a_{k}=0$ para $k \neq i, j$. Daí, $\left(a_{1}, \cdots, a_{n}\right) \in S(2, n)$ e $\prod_{k=1}^{n}\left(P_{k}\right)^{a_{k}}=P_{i} P_{j} \leq K P_{i+j-1}$.

Caso $i=j$, considere $a_{i}=2$ e $a_{k}=0$ para $k \neq i$. Nesse caso, temos $\prod_{k=1}^{n}\left(P_{k}\right)^{a_{k}}=P_{i}^{2} \leq K P_{2 i-1}$.

Portanto,

$$
\frac{P_{i} P_{j}}{P_{i+j-1}} \leq K, \forall i, j \in \mathbb{N}
$$

isto é,

$$
\sup _{i, j \in \mathbb{N}} \frac{P_{i} P_{j}}{P_{i+j-1}} \leq K
$$

A partir da Proposição 3.3.13, temos o seguinte Corolário:

Corolário 3.3.14. (Abtahi-Honary, [2]) Seja $M=\left(M_{n}\right)$ sequência de álgebra tal que d $(M)=0$. Suponha que a sequência $P_{k}=M_{k} / k$ ! seja tal que

$$
K=\sup _{i, j \in \mathbb{N}} \frac{P_{i} P_{j}}{P_{i+j-1}}<\infty .
$$

Então, se $\left(A_{m}\right)$ é a sequência definida em 3.6, temos $\left(A_{m}\right)^{1 / m} \rightarrow 0$.

Demonstração. Pela Proposição 3.3.13, segue que

$$
\frac{1}{P_{n}} \prod_{k=1}^{n}\left(P_{k}\right)^{a_{k}} \leq K^{m-1} \frac{P_{n-m+1}}{P_{n}}, \quad \forall n \geq m, \forall\left(a_{1}, \cdots, a_{n}\right) \in S(m, n) .
$$


Então,

$$
A_{m} \leq \sup _{n \geq m} K^{m-1} \frac{P_{n-m+1}}{P_{n}} \leq \frac{K^{m-1}}{P_{m-1}}, \forall m \in \mathbb{N},
$$

pois $P_{n-m+1} P_{m-1} \leq P_{n}$ (Lema 3.3.10 - 1).

Uma vez que $d(M)=0$, temos $\frac{1}{\left(P_{m-1}\right)^{1 / m-1}} \rightarrow 0$. Por outro lado, como $A_{m} \leq 1, \forall m \in \mathbb{N}$, e pelo fato da função $\left(x \in(0, \infty) \mapsto a^{x}\right), 0<a \leq 1$, ser decrescente, segue que

$$
A_{m}^{1 / m} \leq A_{m}^{1 /(m-1)} \leq\left(\frac{K^{m-1}}{P_{m-1}}\right)^{1 /(m-1)}=\frac{K}{\left(P_{m-1}\right)^{1 / m-1}} \rightarrow 0 .
$$

O Corolário 3.3.14 nos permite mostrar o seguinte resultado:

Corolário 3.3.15. (Abtahi-Honary, [2]) Seja $M=\left(M_{n}\right) \operatorname{com} d(M)=0$. Se $P_{k}=M_{k} / k$ ! é tal que $\left(P_{k}\right)^{2} \leq P_{k-1} P_{k+1}, \forall k \in \mathbb{N}$. Então, se $\left(A_{m}\right)$ é a sequência definida em 3.6, temos $\left(A_{m}\right)^{1 / m} \rightarrow 0$.

Demonstração. Por hipótese,

$$
\frac{P_{i}}{P_{i-1}} \leq \frac{P_{i+1}}{P_{i}} \leq \frac{P_{i+2}}{P_{i+1}} \leq \cdots \leq \frac{P_{i+j}}{P_{i+j-1}}, \quad \forall i, j \geq 1 .
$$

Se $j>i \geq 1$, existe $l$ tal que $j=i+l-1$. Daí,

$$
\frac{P_{i}}{P_{i-1}} \leq \frac{P_{i+l}}{P_{i+l-1}}
$$

implicando que

$$
\frac{P_{i}}{P_{i-1}} \leq \frac{P_{j+1}}{P_{j}}
$$

Logo, $P_{i} P_{j} \leq P_{i-1} P_{j+1}, \forall i, j \geq 1$, pois o caso $i=j$ é a hipótese do corolário. Assim,

$$
P_{i} P_{j} \leq P_{i-1} P_{j+1} \leq P_{i-2} P_{j+2} \leq \cdots \leq P_{1} P_{i+j-1} .
$$

Portanto,

$$
\sup _{i, j \in \mathbb{N}} \frac{P_{i} P_{j}}{P_{i+j-1}} \leq P_{1}<\infty
$$

Concluímos do Corolário 3.3.14 que $\left(A_{m}\right)^{1 / m} \rightarrow 0$.

A partir do Corolário 3.3.15, conseguimos apresentar exemplos de sequências de álgebras que satisfazem a condição $\left(A_{m}\right)^{1 / m} \rightarrow 0$.

Exemplo 3.3.16. Sejam $M_{0}=1$ e $M_{n}=n !^{\alpha}$, onde $\alpha>1$. Daí, $P_{0}=1$ e $P_{n}=n !^{\alpha-1}$. Como $n \leq n+1$, temos

$$
(n-1) ! n n ! \leq(n-1) !(n+1) n ! \Rightarrow n !^{2} \leq(n-1) !(n+1) ! \Rightarrow\left(n !^{2}\right)^{\alpha} \leq((n-1) !(n+1) !)^{\alpha} .
$$

Portanto, $\left(P_{k}\right)^{2} \leq P_{k-1} P_{k+1}, \forall k \in \mathbb{N}$.

O próximo Lema será utilizado para apresentar o Exemplo 3.3.18.

Lema 3.3.17. Seja $\left(P_{k}\right)$ sequência de números reais positivos tal que

$$
P_{0}=1 e\left(P_{k}\right)^{2} \leq P_{k-1} P_{k+1}, \forall k .
$$

Então, se $M=\left(n ! P_{n}\right), M$ é sequência de álgebra. 
Demonstração. Assim como no Corolário 3.3.15, temos $P_{i} P_{j} \leq P_{i-1} P_{j+1}, \forall i, j \geq 1$. Daí,

$$
P_{i} P_{j} \leq P_{i-1} P_{j+1} \leq \cdots \leq P_{0} P_{i+j}=P_{i+j} .
$$

Assim, se $n=i+j$ e $k=i$, temos

$$
\frac{P_{n}}{P_{k} P_{n-k}} \geq 1
$$

Donde,

$$
\frac{M_{n}}{M_{k} M_{n-k}} \geq\left(\begin{array}{l}
n \\
k
\end{array}\right)
$$

Exemplo 3.3.18. Sejam $M_{0}=1$ e $M_{n}=n$ ! $[\log (n+1)]^{n}$. Assim, $P_{0}=1$ e $P_{n}=[\log (n+1)]^{n}$. Queremos provar que $\frac{P_{n}}{P_{n-1}} \leq \frac{P_{n+1}}{P_{n}}, \forall n \in \mathbb{N}$. Com efeito, considere a função $f(x)=x \log (\log (x+1))$. Derivando $f$ duas vezes, temos

$$
f^{\prime \prime}(x)=\frac{1}{(x+1)^{2}(\log (x+1))^{2}}((x+2) \log (x+1)-x) \geq 0, \forall x \geq 0 .
$$

Logo, $f$ é convexa. Daí, $2 f(n) \leq f(n-1)+f(n+1)$, i.e

$$
2 n \log (\log (n+1)) \leq(n-1) \log (\log (n))+(n+1) \log (\log (n+2)) .
$$

Utilizando propriedades básicas de logaritmo, segue que

$$
\left(\log (\log (n+1))^{2 n}\right) \leq \log \left((\log (n))^{n-1}(\log (n+2))^{n+1}\right) .
$$

Usando que a função log é crescente, segue que $(\log (n+1))^{2 n} \leq(\log (n))^{n-1}(\log (n+2))^{n+1}$. Então $\left(P_{k}\right)^{2} \leq P_{k-1} P_{k+1}, \forall k \in \mathbb{N}$.

Daí, pelo Lema 3.3.17, temos que $M$ é uma sequência de álgebra. Além disso, como

$$
d(M)=\lim _{n \rightarrow \infty}\left[\frac{1}{(\log (n+1))^{n}}\right]^{1 / n}=\lim _{n \rightarrow \infty} \frac{1}{\log (n+1)}=0,
$$

concluímos pelo Corolário 3.3.15 que $A_{m}^{1 / m} \rightarrow 0$.

Os Exemplos 3.3.16 e 3.3.18 são exemplos de sequências de álgebras que satisfazem $A_{m}^{1 / m} \rightarrow 0$. Por conseguinte, se $X$ é uniformemente regular, teremos, nesses casos, que $D(X, M)$ será uma álgebra natural.

Recordemos que se $\left(a_{n}\right)$ uma sequência limitada de números reais positivos. Então,

$$
\liminf _{n \rightarrow \infty} \frac{a_{n+1}}{a_{n}} \leq \liminf _{n \rightarrow \infty}\left(a_{n}\right)^{1 / n} \leq \limsup _{n \rightarrow \infty}\left(a_{n}\right)^{1 / n} \leq \limsup _{n \rightarrow \infty} \frac{a_{n+1}}{a_{n}} .
$$

A desigualdade acima é estudada em cursos de nível de graduação de análise real cuja demonstração pode ser encontrada em [24].

A seguir, apresentaremos uma outra condição apresentada por Abtahi e Honary que implica em $A_{m}^{1 / m} \rightarrow 0$.

Proposição 3.3.19. (Abtahi - Honary, [2]) Seja $\left(P_{k}\right)$ sequência de números reais positivos tais que $P_{i} P_{j} \leq P_{i+j}, \forall i, j \in \mathbb{N}$. Defina, para cada $n \in \mathbb{N}$,

$$
B_{n}=\max \left\{\frac{P_{k} P_{n-k}}{P_{n}}: 1 \leq k \leq n-1\right\} .
$$

Se $B_{n} \rightarrow 0$ e se $\left(A_{m}\right)$ é dada como em 3.6, segue que $\left(A_{m}\right)^{1 / m} \rightarrow 0$. 
Demonstração. Como $\left(A_{m}\right)$ é uma sequência limitada de números positivos, temos

$$
\liminf _{n \rightarrow \infty} \frac{A_{n+1}}{A_{n}} \leq \liminf _{n \rightarrow \infty}\left(A_{n}\right)^{1 / n} \leq \limsup _{n \rightarrow \infty}\left(A_{n}\right)^{1 / n} \leq \limsup _{n \rightarrow \infty} \frac{A_{n+1}}{A_{n}} .
$$

Assim, é suficiente verificar que $A_{m+1} / A_{m} \rightarrow 0$. Uma vez que $B_{n} \rightarrow 0$, dado $\epsilon>0$, existe $N \in \mathbb{N}$ tal que $B_{n}<\epsilon, \forall n \geq N$. Daí,

$$
\frac{P_{i} P_{n-i}}{P_{n}}<\epsilon, \quad \forall n \geq N, \forall i=1, \cdots, n-1 .
$$

Sejam $n \geq m+1$ e $\left(a_{1}, \cdots, a_{n}\right) \in S(m+1, n)$. Então, $\sum_{k=1}^{n} a_{k}=m+1$ e $\sum_{k=1}^{n} k a_{k}=n$. Se $a_{1}=\cdots=a_{n-1}=0$, teríamos $a_{n}=m+1$. Logo, $n(m+1)=n$, que implicaria que $m+1=0$, contradição. Logo, existe $i \in\{1, \cdots, n-1\}$ tal que $a_{i} \geq 1$. Desse modo,

$$
\left\{\begin{array}{c}
a_{1}+\cdots+a_{i-1}+\left(a_{i}-1\right)+a_{i+1}+\cdots+a_{n}=m \\
a_{1}+\cdots+(i-1) a_{i-1}+i\left(a_{i}-1\right)+(i+1) a_{i+1}+\cdots+n a_{n}=n-i
\end{array}\right.
$$

Em particular, temos que $n-i \geq m$.

Se para algum $k>n-i, a_{k}>0$, teríamos $n \geq i+k>n$, contradição. Assim, $a_{k}=0, \forall k>n-i$. Daí, se $b_{k}=a_{k}$ para $k=1, \cdots, i-1, i+1, \cdots, n-i$, e $b_{i}=a_{i}-1$, temos $\left(b_{1}, \cdots, b_{n-i}\right) \in S(m, n-i)$. Então,

$$
\frac{1}{P_{n-i}} \prod_{k=1}^{n-i}\left(P_{k}\right)^{b_{k}} \leq A_{m}
$$

No entanto,

$$
\frac{1}{P_{n-i}} \prod_{k=1}^{n-i}\left(P_{k}\right)^{b_{k}}=\frac{1}{P_{n-i}} \prod_{k=1}^{n}\left(P_{k}\right)^{a_{k}}\left(P_{i}\right)^{-1}
$$

Assim,

$$
\frac{1}{P_{n}} \prod_{k=1}^{n}\left(P_{k}\right)^{a_{k}}=\frac{P_{n-i} P_{i}}{P_{n}} \frac{1}{P_{n-i}} \prod_{k=1}^{n}\left(P_{k}\right)^{a_{k}}\left(P_{i}\right)^{-1}<\epsilon A_{m} .
$$

Isto é,

$$
\frac{1}{P_{n}} \prod_{k=1}^{n}\left(P_{k}\right)^{a_{k}}<\epsilon A_{m}, \forall n \geq m+1, \forall\left(a_{1}, \cdots, a_{n}\right) \in S(m+1, n) .
$$

Logo,

$$
A_{m+1}<\epsilon A_{m}, \forall m \geq N
$$

Portanto, $A_{m+1} / A_{m} \rightarrow 0$.

A partir do Teorema 3.3.12 e da Proposição 3.3.19, temos o seguinte resultado:

Corolário 3.3.20. (Abtahi-Honary, [2]) Sejam $X$ uniformemente regular, $M=\left(M_{n}\right)$ sequência de álgebra, $P_{n}=M_{n} / n$ ! e $B_{n}$ dado em 3.10. Então, se $B_{n} \rightarrow 0, D(X, M)$ é álgebra natural.

A Proposição 3.3.21 mostrará quais tipos de sequências satisfazem $B_{n} \rightarrow 0$.

Proposição 3.3.21. (Abtahi-Honary, [2]) Se $M=\left(M_{n}\right)$ é uma sequência de álgebra tal que d $(M)=$ 0 e $P_{n}=M_{n} / n$ ! satisfaz $P_{n}^{2} \leq P_{n-1} P_{n+1}, \forall n \in \mathbb{N}$. Então, $B_{n} \rightarrow 0$.

Demonstração. Como $P_{n} / P_{n+1} \leq P_{n-1} / P_{n}, \forall n \in \mathbb{N}$ e $d(M)=0$, temos $P_{n}^{1 / n} \rightarrow \infty$. Por 3.9, segue que $P_{n} / P_{n-1} \rightarrow \infty$, $\operatorname{logo} P_{n-1} / P_{n} \rightarrow 0$. A partir da demonstração do Corolário 3.3 .15 , concluímos que $K=\sup _{i, j \in \mathbb{N}} \frac{P_{i} P_{j}}{P_{i+j-1}}<\infty$. Daí, para $n \in \mathbb{N}$ e $k=1, \cdots, n-1$, temos

$$
K \geq \frac{P_{k} P_{n-k}}{P_{n-1}}=\frac{P_{k} P_{n-k}}{P_{n-1}} \frac{P_{n}}{P_{n}}=\frac{P_{k} P_{n-k}}{P_{n}} \frac{P_{n}}{P_{n-1}},
$$


o que implica que

$$
\frac{P_{n-1}}{P_{n}} K \geq \frac{P_{k} P_{n-k}}{P_{n}}
$$

Logo, $B_{n} \leq K\left(P_{n-1} / P_{n}\right), \forall n \in \mathbb{N}$. Em suma, concluímos que $B_{n} \rightarrow 0$.

A partir da Proposição 3.3.21, concluímos que as sequências de álgebra vistas nos exemplos 3.3.16 e 3.3.18 são tais que $B_{n} \rightarrow 0$.

Tendo em vista o que foi feito nesta seção, temos que se $X$ é uniformemente regular, $M=\left(M_{n}\right)$ é sequência de álgebra e $P_{n}=M_{n} / n$ !, então:

1. se $d(M)=0$ e $\sup _{i, j \in \mathbb{N}} \frac{P_{i} P_{j}}{P_{i+j-1}}<\infty$, temos que $D(X, M)$ é natural.

2. se $B_{n}=\max _{k=1, \cdots, n-1} \frac{P_{k} P_{n-k}}{P_{n}} \rightarrow 0$, temos que $D(X, M)$ é natural.

Além disso,

$$
d(M)=0 \text { e } P_{n}^{2} \leq P_{n-1} P_{n+1}, \forall n \in \mathbb{N} \text {, o que implica que } D(X, M) \text { é natural. }
$$

Em [2], Abtahi e honary deixaram o seguinte pergunta em aberto:

Problema 3.3.22. Se $X$ é um conjunto uniformemente regular e se $\left(M_{n}\right)$ é uma sequência de álgebra tal que $d(M)=0$, então a álgebra $D(X, M)$ é natural?

Como vimos nessa seção, Abtahi e Honary mostraram que se $d(M)=0$ e $P_{n}^{2} \leq P_{n-1} P_{n+1}$, então $D(X, M)$ será natural. O Lema 3.3.17 mostra que se $\left(P_{n}\right)$ é sequência de números reais positivos satisfazendo $P_{n}^{2} \leq P_{n-1} P_{n+1}$, então a sequência $M=\left(M_{n}\right)$ tal que $M_{n}=P_{n} n$ ! é uma sequência de álgebra. Entretanto, essa relação não implica em $d(M)=0$ como mostra o próximo Exemplo:

Exemplo 3.3.23. Se $M_{n}=n$ !, temos $P_{n}=1, \forall n \in \mathbb{N}_{0}$. Assim, $P_{n}^{2}=1=P_{n-1} P_{n+1}$ e $d(M)=1$.

Uma pergunta natural é se $d(M)=0$ implica na relação $P_{n}^{2} \leq P_{n-1} P_{n+1}$. Caso a afirmação seja verdadeira, o Problema 3.3.22 estaria respondido. 


\section{Capítulo 4}

\section{Lineabilidade e Algebrabilidade em um Subconjunto da Álgebra de Disco}

Se $X=\overline{\mathcal{D}}$ e $M=\left(M_{n}\right)$ é uma sequência de álgebra, temos que $D(\overline{\mathcal{D}}, M)$ está contido em $\mathcal{A}(\mathcal{D})$. Uma pergunta natural é quão distante algébrica e topologicamente estão estas álgebras.

A resposta para esta pergunta está dentro do tema de algebrabilidade e residualidade sobre determinados conjuntos. Com este enfoque, Lourenço e Vieira estudaram a diferença entre esses conjuntos nos textos [25, 26]. Neste capítulo, vamos estudar os textos científicos [25, 26].

Recordemos que

$$
f \in D(\overline{\mathcal{D}}, M) \Longleftrightarrow f \in D^{\infty}(\overline{\mathcal{D}}) \text { e } \sum_{n=0}^{\infty} \frac{1}{M_{n}}\left\|f^{(n)}\right\|_{\overline{\mathcal{D}}}<\infty .
$$

No que segue, vamos estudar o conjunto:

$$
\mathcal{H}(M)=\mathcal{A}(\mathcal{D}) \backslash D(\overline{\mathcal{D}}, M) .
$$

Estaremos interessados em sequências de álgebras tais que $d(M) \neq 0$, pois, neste caso $R_{0}(\overline{\mathcal{D}})$ não está contido em $D(\overline{\mathcal{D}}, M)$ e, por conseguinte, $\mathcal{H}(M) \neq \emptyset$. Quando $M_{n}=n$ !, escreveremos $\mathcal{H}$ ao invés de $\mathcal{H}(M)$.

Os resultados abordados neste capítulo podem ser encontrados em [3, 25, 26].

\subsection{Preliminares}

Nesta seção, trataremos a respeito dos pré-requisitos para este capítulo. As definições e resultados encontrados nesta seção podem ser encontrados no capítulo 1 do livro [3].

A seguir vamos definir lineabilidade de um subconjunto num espaço vetorial.

Definição 4.1.1. Sejam $E$ um espaço vetorial, $S \subset E$ e $\mu$ um número cardinal. Dizemos que $S$ é $\mu$-lineável se $S \cup\{0\}$ contém um espaço vetorial de dimensão $\mu$. Caso o respectivo espaço vetorial contido em $S \cup\{0\}$ tenha dimensão infinita, vamos nos referir simplesmente como lineável.

Recordemos que um espaço vetorial topológico é um espaço vetorial munido de uma topologia tal que as operações de adição e de multiplicação por escalar são contínuas. Em subconjuntos de espaços vetoriais topológicos podemos definir o conceito de espaçabilidade.

Definição 4.1.2. Sejam $E$ um espaço vetorial topológico, $S \subset E$ e $\mu$ um número cardinal. Dizemos que $S$ é $\mu$-espaçável se $S \cup\{0\}$ contém um espaço vetorial fechado de dimensão $\mu$. Caso o respectivo espaço vetorial contido em $S \cup\{0\}$ tenha dimensão infinita, vamos nos referir simplesmente como espaçável. 
É imediato das definições que se $S$ é um subconjunto espaçável de um espaço vetorial topológico $V$, então $S$ é lineável.

A noção de algebrabilidade segue da mesma ideia da lineabilidade, só que para álgebras. Antes de apresentarmos a definição de algebrabilidade, precisamos falar a respeito de sistema de geradores de uma álgebra e independência algébrica.

Se $S$ é um subconjunto de uma álgebra (unital) $\mathcal{A}$, definimos a álgebra gerada por $S$, denotada por $\mathcal{A}(S)$, pela intersecção de todas as subálgebras (unitais) de $\mathcal{A}$ que contém $S$. Um conjunto $S=\left\{z_{i}: i \in I\right\}$ é um sistema de geradores para $\mathcal{A}$ se $\mathcal{A}=\mathcal{A}(S)$. Além disso, se para todo $i_{0} \in I, z_{i_{0}} \notin \mathcal{A}\left(S \backslash\left\{i_{0}\right\}\right), S$ é um sistema minimal de geradores.

Ademais, o conjunto $S$ é dito livre ou algebricamente independente se

$$
\left(P\left(z_{i_{1}}, \cdots, z_{i_{n}}\right)=0 \Rightarrow P=0\right), \forall P \in \mathbb{C}\left[z_{1}, \cdots, z_{n}\right], \forall z_{i_{1}}, \cdots, z_{i_{n}} \in S .
$$

Em particular, se $a$ e $b$ são algebricamente independentes em $\mathcal{A}$, a aplicação $\Phi: \mathbb{C}\left[z_{1}, z_{2}\right] \rightarrow$ $\mathcal{A}(\{a, b\})$ dada por $\Phi(P)=P(a, b)$ é um isomorfismo. De fato, é imediato que $\Phi$ é um homomorfismo de álgebras. A sobrejetividade segue do fato de todo elemento de $\mathcal{A}(\{a, b\})$ ser da forma $\sum_{j, k=1}^{m} \alpha_{j k} a^{j} b^{k}$. Enquanto a injetividade é consequência do fato de $a$ e $b$ serem algebricamente independentes.

Exemplo 4.1.3. Sejam $g_{1}(z)=e^{z}$ e $g_{2}(z)=e^{\beta z}$, onde $\beta \in \mathbb{R} \backslash \mathbb{Q}$. Vamos mostrar que $g_{1}$ e $g_{2}$ são algebricamente independentes. Com efeito, seja $P\left(z_{1}, z_{2}\right)=\sum_{j, k=0}^{m} \alpha_{j k} z_{1}^{j} z_{2}^{k}$ um polinômio em $\mathbb{C}\left[z_{1}, z_{2}\right]$ tal que $P\left(g_{1}, g_{2}\right)=0$. Daí,

$$
0=\sum_{j, k=0}^{m} \alpha_{j k}\left(e^{z}\right)^{j}\left(e^{\beta z}\right)^{k}=\sum_{j, k=0}^{m} \alpha_{j k} e^{(j+\beta k) z}=\sum_{j, k=0}^{m} \alpha_{j k} e^{\beta_{j k} z}, \forall z \in \mathbb{C}, \quad \beta_{j k}=j+\beta k .
$$

Derivando a equação acima $r$ vezes e aplicando em $z=1$, segue que $\sum_{j, k=0}^{m} \alpha_{j k} \beta_{j k}^{r} e^{\beta_{j k}}=0$. Ilustraremos aqui o caso $m=2$, o caso geral é análogo. De fato, como $\beta_{00}=0$, temos o seguinte sistema:

$$
\left[\begin{array}{cccc}
\beta_{01} e^{\beta_{01}} & \beta_{02} e^{\beta_{02}} & \cdots & \beta_{22} e^{\beta_{22}} \\
\beta_{01}^{2} e^{\beta_{01}} & \beta_{02}^{2} e^{\beta_{02}} & \cdots & \beta_{22}^{2} e^{\beta_{22}} \\
\vdots & \vdots & \ddots & \vdots \\
\beta_{01}^{8} e^{\beta_{01}} & \beta_{02}^{8} e^{\beta_{02}} & \cdots & \beta_{22}^{8} e^{\beta_{22}}
\end{array}\right]\left[\begin{array}{c}
\alpha_{01} \\
\alpha_{02} \\
\vdots \\
\alpha_{22}
\end{array}\right]=\left[\begin{array}{c}
0 \\
0 \\
\vdots \\
0
\end{array}\right] .
$$

O determinante da matriz dos coeficientes do sistema acima é

$$
e^{\beta_{01}} e^{\beta_{02}} \cdots e^{\beta_{22}} \beta_{01} \beta_{02} \cdots \beta_{22} \prod\left(\beta_{j k}-\beta_{p q}\right),
$$

onde $j, k, p, q=0,1,2,(j, k) \neq(p, q),(j, k) \neq(0,0),(p, q) \neq(0,0)$.

Como os $e^{\beta_{j k}} \neq 0$ e $\beta_{j k} \neq 0$, temos que o determinante é igual a zero se, e só se, $\beta_{j k}=\beta_{p q}$ para certos $(j, k) \neq(p, q)$. Não obstante, teríamos $j+\beta k=p+\beta q$. Assim, pela irracionalidade de $\beta,(j, k) \neq(p, q)$, uma contradição. Então, $\alpha_{j k}=0, \forall j, k$ e $(j, k) \neq(0,0)$. Logo, $P=\alpha_{00}$ e como $P\left(g_{1}, g_{2}\right)=0$, concluímos que $P=0$.

A partir das observações acima, temos a seguinte definição:

Definição 4.1.4. Sejam $\mathcal{A}$ uma álgebra de Banach, $M \subset \mathcal{A}$ e $\alpha$ e $\beta$ dois cardinais. Dizemos que $M$ é

1. algebrável se existe subálgebra $\mathcal{C}$ de $\mathcal{A}$ contida em $M \cup\{0\}$ tal que cardinalidade de qualquer sistema de geradores de $\mathcal{C}$ é infinita.

2. ( $\alpha, \beta)$-algebrável se existe subálgebra $\mathcal{C}$ de $\mathcal{A}$ contida em $M \cup\{0\}$ tal que $\operatorname{dim}(\mathcal{C})=\alpha$ e card $(S)=\beta$, onde $S$ é um sistema minimal de geradores para $\mathbb{C}$. 
3. fortemente $\alpha$-algebrável se existe uma subálgebra $\mathcal{C}$ de $\mathcal{A}$ contida em $M \cup\{0\}$ que admite um sistema de geradores livre com cardinalidade $\alpha$.

Existem outras definições dentro dos tópicos de lineabilidade e algebrabilidade. No entanto, enunciamos apenas as que serão utilizadas neste trabalho. No livro [3], os autores enunciam todas as definições nesta área.

Na literatura, encontramos algumas técnicas que auxiliam na demonstração de que um conjunto é lineável, espaçável, algebrável ou fortemente $\alpha$-algebrável. A seguir, enunciaremos alguns resultados, sem apresentar a demonstração, que serão utilizados neste capítulo. O próximo resultado foi originalmente enunciado para Espaços de Fréchet em [12] e utilizado por Kitson e Timoney em [21]. Como não abordaremos esses espaços nesta dissertação, enunciaremos o resultado para espaços de Banach, uma vez que todo espaço de Banach é um espaço de Fréchet.

Teorema 4.1.5. Sejam E e $F$ espaços de Banach e $T: F \rightarrow E$ um operador linear contínuo cuja imagem não é fechada. Então, existe um subespaço vetorial fechado de E com dimensão infinita contido em $(E \backslash T(F)) \cup\{0\}$, isto é, $E \backslash T(F)$ é espaçável.

Denotaremos por $\mathcal{E}$ a família das funções de $\mathbb{C} \rightarrow \mathbb{C}$ da forma

$$
\varphi(z)=\sum_{j=1}^{m} a_{j} e^{b_{j} z},
$$

onde $a_{1}, \cdots, a_{m}, b_{1}, \cdots, b_{m} \in \mathbb{C} \backslash\{0\}$ e os $b_{j}^{\prime} s$ são dois a dois distintos.

Com a notação acima, apresentamos um Teorema cuja demonstração pode ser encontrado na página 270 do livro [3].

Teorema 4.1.6. Sejam $\Omega$ um conjunto não vazio e $\mathcal{F}$ uma família de funções de $\Omega \rightarrow \mathbb{K}$, onde $\mathbb{K}=\mathbb{R}$ ou $\mathbb{K}=\mathbb{C}$. Suponha que exista uma função $f \in \mathcal{F}$ cuja imagem $f(\Omega)$ é não enumerável $e$ $\varphi \circ f \in \mathcal{F}, \forall \varphi \in \mathcal{E}$. Então, $\mathcal{F}$ é fortemente c-algebrável. Mais precisamente, se $H \subset(0, \infty)$ é um conjunto com cardinalidade $\mathfrak{c}$ e linearmente independente sobre o corpo $\mathbb{Q}$, então

$$
\{\exp \circ(r f): r \in H\}
$$

é um sistema de geradores livre para uma álgebra contida em $\mathcal{F} \cup\{0\}$.

\subsection{Lineabilidade e Espaçabilidade}

Nesta seção, vamos estudar a lineabilidade do conjunto $\mathcal{H}(M)$ definido em 4.1. Mais precisamente, considerando $M_{n}=n$ ! , temos que $d(M) \neq 0$ e, pelo Teorema 3.3.3, segue que $R_{0}(\overline{\mathcal{D}})$ não está contido em $D(\overline{\mathcal{D}}, M)$. Desse modo, veremos que $\mathcal{H}$ é não vazio.

É imediato que espaçabilidade implica em lineabilidade, entretanto como os resultados foram provados utilizando técnicas independentes, vamos apresentar os resultados de [25, 26], onde Lourenço e Vieira mostraram que $\mathcal{H}$ é $\aleph_{0}$-lineável, c-lineável e espaçável.

O próximo Lema apresenta uma classe de funções que está contida no conjunto $\mathcal{H}$.

Lema 4.2.1. (Lourenço - Vieira, [25]) Sejam $w=2 e^{i \theta}$, onde $0 \leq \theta \leq 2 \pi$, e $f(z)=\frac{1}{z-w}, \forall z \in \overline{\mathcal{D}}$. Então, $f \in \mathcal{H}$.

Demonstração. Por definição, $f$ é uma função racional com polo fora de $\overline{\mathcal{D}}, \operatorname{logo} f \in \mathcal{A}(\mathcal{D})$. Além disso, pelo Princípio do Máximo - 2.1.3:

$$
\|f\|_{\overline{\mathcal{D}}}=\sup _{z \in \overline{\mathcal{D}}}|f(z)|=\max _{0 \leq \alpha \leq 2 \pi} \frac{1}{\left|e^{i \alpha}-2 e^{i \theta}\right|}=\min _{0 \leq \alpha \leq 2 \pi}\left|e^{i \alpha}-2 e^{i \theta}\right|=1 .
$$


Como $f^{(n)}=(-1)^{n} n ! f^{n+1}(z), z \in \overline{\mathcal{D}}$, temos $\left\|f^{(n)}\right\|_{\overline{\mathcal{D}}}=n !\|f\|_{\overline{\mathcal{D}}}^{n+1}=n !$ Então,

$$
\sum_{n=0}^{\infty} \frac{1}{M_{n}}\left\|f^{(n)}\right\|_{\overline{\mathcal{D}}}=\sum_{n=0}^{\infty} 1=+\infty
$$

Donde, $f \in \mathcal{H}$.

Se $f \in C(K)$ e $n \in \mathbb{N}$, define-se a função $f^{n}: K \rightarrow \mathbb{C}$ por $f^{n}(z)=(f(z))^{n}$, para cada $z \in K$. Utilizando esta notação, vamos apresentar a demonstração de que $\mathcal{H}$ é $\aleph_{0}$-lineável.

Proposição 4.2.2. (Lourenço - Vieira, [25]) O conjunto $\mathcal{H}$ é $\aleph_{0}$-lineável.

Demonstração. Pelo Lema 4.2.1, temos que $f(z)=\frac{1}{z-2} \in \mathcal{H}$. Considere $S=\left\{f^{n}: n \in \mathbb{N}\right\}$.

Como $f \in \mathcal{A}(\mathcal{D})$ e $\mathcal{A}(\mathcal{D})$ é uma álgebra, $f^{n} \in \mathcal{A}(\mathcal{D}), \forall n$. Logo, $S \subset \mathcal{A}(\mathcal{D})$ e, por conseguinte, $[S] \subset \mathcal{A}(\mathcal{D})$. Vamos provar que $[S] \subset \mathcal{H} \cup\{0\}$.

Vejamos primeiro que $S$ é linearmente independente. Com efeito, sejam $\beta_{1}, \cdots, \beta_{k} \in \mathbb{C}$ tais que $\sum_{j=1}^{k} \beta_{j} f^{j}=0$. Em particular, dados $z_{1}, \cdots, z_{k} \in \mathcal{D} \cap \mathbb{R}$ dois a dois distintos, temos que $f\left(z_{1}\right), \cdots, f\left(z_{k}\right) \in \mathbb{R}$ são dois a dois distintos. Logo, $\sum_{j=1}^{k} \beta_{j} f^{j}\left(z_{i}\right)=0, \forall i=1, \cdots, k$. Em notação matricial:

$$
\left[\begin{array}{cccc}
f\left(z_{1}\right) & f\left(z_{1}\right)^{2} & \cdots & f\left(z_{1}\right)^{k} \\
f\left(z_{2}\right) & f\left(z_{2}\right)^{2} & \cdots & f\left(z_{2}\right)^{k} \\
\vdots & \vdots & \ddots & \vdots \\
f\left(z_{k}\right) & f\left(z_{k}\right)^{2} & \cdots & f\left(z_{k}\right)^{k}
\end{array}\right]\left[\begin{array}{c}
\beta_{1} \\
\beta_{2} \\
\vdots \\
\beta_{k}
\end{array}\right]=\left[\begin{array}{c}
0 \\
0 \\
\vdots \\
0
\end{array}\right] .
$$

A matriz dos coeficientes do sistema acima é uma matriz de Vandermonde e portanto tem determinante igual:

$$
f\left(z_{1}\right) \cdots\left(z_{k}\right) \prod_{1 \leq i<j \leq k}\left(f\left(x_{i}\right)-f\left(x_{j}\right)\right) \neq 0 .
$$

Assim, temos um sistema homogêneo possível e determinando, $\operatorname{logo} \beta_{1}=\cdots=\beta_{k}=0$ e, consequentemente, $S$ é linearmente independente.

Queremos agora mostrar que $[S] \subset \mathcal{H} \cup\{0\}$. Para isso, seja $g \in[S]$, veremos que se $g \neq 0$, então $g \in \mathcal{H}$. De fato, se $g \neq 0$, existem $\beta_{1}, \cdots, \beta_{k} \in \mathbb{C}$ não todos nulos tais que $g=\sum_{j=1}^{k} \beta_{j} f^{j}$. Mas como,

$$
\left(f^{j}\right)^{(n)}=(-1)^{n} j(j+1) \cdots(j+n-1) f^{j+n} \text { e } f(1)=-1,
$$

segue que

$$
g^{(n)}(1)=\sum_{j=1}^{n} \beta_{j}(-1)^{n} j(j+1) \cdots(j+n-1)(-1)^{j+n}=\sum_{j=1}^{n} \beta_{j}(-1)^{j} \frac{(j+n-1) !}{(j-1) !} .
$$

Logo,

$$
\frac{g^{(n)}(1)}{n !}=-\beta_{1} \frac{1}{0 !}+\beta_{2} \frac{n+1}{1 !}-\beta_{3} \frac{(n+1)(n+2)}{2 !}+\cdots+(-1)^{k} \beta_{k} \frac{(n+1) \cdots(n+k-1)}{(k-1) !} .
$$

Por outro lado, se $\sum_{n=0}^{\infty} \frac{\left|g^{(n)}(1)\right|}{n !}<\infty$, temos que $\lim _{n \rightarrow \infty} \frac{\left|g^{(n)}(1)\right|}{n !} \rightarrow 0$, então $\beta_{1}=\cdots=\beta_{k}=0$, contradição. Logo, $g \in \mathcal{H}$ e, por conseguinte, $[S] \subset \mathcal{H} \cup\{0\}$. Em suma, $\mathcal{H}$ é $\aleph_{0}$-lineável.

Com a mesma notação da Proposição 4.2.2, temos, em particular, que a álgebra gerada pelo conjunto $S$ coincide com o espaço gerado por $S$, i.e $\mathcal{A}(S)=[S]$. De fato, é imediato que $[S] \subset \mathcal{A}(S)$. Por outro lado, $[S]$ também é uma subálgebra de $\mathcal{A}(\mathcal{D})$ que contém $S, \operatorname{logo} \mathcal{A}(S) \subset[S]$.

Desse modo, como $\operatorname{dim}(\mathcal{A}(S))=\operatorname{dim}([S])=\aleph_{0}$ e $\{f\}$ é um sistema minimal de geradores para $\mathcal{A}(S)$, temos o seguinte Corolário: 
Corolário 4.2.3. (Lourenço - Vieira, [25]) O conjunto $\mathcal{H}$ é $\left(\aleph_{0}, 1\right)$-algebrável.

Na Proposição 4.2.2, Lourenço e Vieira apresentaram um espaço vetorial de dimensão $\aleph_{0}$ contido em $\mathcal{H}$. Em [26], Lourenço e Vieira demonstraram que $\mathcal{H}$ contém um espaço vetorial de dimensão $\mathfrak{c}$. Para isso, fixaremos algumas notações. Dados $\alpha \in(0,1)$ e $f \in \mathcal{A}(\mathcal{D})$, definimos a função $f_{\alpha}: \overline{\mathcal{D}} \rightarrow \mathbb{C}$ por $f_{\alpha}(z)=f(\alpha z)$, para todo $z \in \mathcal{D}$. Uma vez que $f_{\alpha}$ é a composição das funções $f$ e $(z \in \overline{\mathcal{D}} \rightarrow \alpha z)$, segue que $f_{\alpha} \in \mathcal{A}(\mathcal{D})$.

Vamos utilizar o próximo Lema, na demonstração de que $\mathcal{H}$ é $\mathfrak{c}$-lineável.

Lema 4.2.4. (Lourenço - Vieira, [26]) Se $f \in \mathcal{A}(\mathcal{D})$ não é um polinômio, então o conjunto $\left\{f_{\alpha}: 0<\alpha<1\right\}$ é linearmente independente.

Demonstração. Sejam $c_{1}, \cdots, c_{N} \in \mathbb{C}$ e $0<\alpha_{1}, \cdots, \alpha_{N}<1$ tais que $\sum_{k=1}^{N} c_{k} f_{\alpha_{k}}=0$. Sem perda de generalidade, suponha $\alpha_{1}<\alpha_{2}<\cdots<\alpha_{N}$. Por hipótese, como $f \in \mathcal{A}(\mathcal{D})$, então $f(z)=\sum_{n=0}^{\infty} a_{n} z^{n}$ uniformemente em $\delta \mathcal{D}=\{\delta z: z \in \mathcal{D}\}$, onde $0<\delta<1$. Daí,

$$
0=\sum_{k=1}^{N} c_{k} f_{\alpha_{k}}(z)=\sum_{k=1}^{N} c_{k} f\left(\alpha_{k} z\right)=\sum_{k=1}^{N} c_{k} \sum_{n=0}^{\infty} a_{n}\left(\alpha_{k} z\right)^{n}=\sum_{n=0}^{\infty}\left(a_{n} \sum_{k=1}^{N} c_{k} \alpha_{k}^{n}\right) z^{n}, \forall z \in \delta \mathcal{D} .
$$

Como a série converge uniformemente em $\delta \mathcal{D}$, segue que

$$
a_{n}\left(c_{1} \alpha_{1}^{n}+\cdots+c_{N} \alpha_{N}^{n}\right)=0, \forall n \in \mathbb{N}_{0} .
$$

Por outro lado, como $f$ não é um polinômio, exite uma sequência crescente $\left(n_{j}\right)_{j \in \mathbb{N}_{0}}$ tal que $a_{n_{j}} \neq$ $0, \forall j \in \mathbb{N}_{0}$. Em particular,

$$
c_{1} \alpha_{1}^{n_{j}}+\cdots+c_{N} \alpha_{N}^{n_{j}}=0, \forall j \in \mathbb{N}_{0}
$$

Se $c_{N} \neq 0$, temos:

$$
-1=\frac{c_{1} \alpha_{1}^{n_{j}}+\cdots+c_{N-1} \alpha_{N-1}^{n_{j}}}{c_{N} \alpha_{N}^{n_{j}}}=\sum_{k=1}^{N-1} \frac{c_{k}}{c_{N}}\left(\frac{\alpha_{k}}{\alpha_{N}}\right)^{n_{j}}, \forall j \in \mathbb{N}_{0} .
$$

Tomando $j \rightarrow+\infty$, segue que $n_{j} \rightarrow+\infty$. Donde, $\left(\frac{\alpha_{k}}{\alpha_{N}}\right)^{n_{j}} \rightarrow 0, \forall k=1, \cdots, N-1$, uma vez que $\alpha_{k}<\alpha_{N}, \forall k=1, \cdots, N-1$. Então,

$$
-1=\lim _{j \rightarrow+\infty} \sum_{k=1}^{N-1} \frac{c_{k}}{c_{N}}\left(\frac{\alpha_{k}}{\alpha_{N}}\right)^{n_{j}}=0, \text { contradição. }
$$

Então, $c_{N}=0$ e $c_{1} \alpha_{1}^{n_{j}}+\cdots+c_{N-1} \alpha_{N-1}^{n_{j}}=0$, para todo $j \in \mathbb{N}_{0}$. Repetindo o argumento para $c_{N-1}, c_{N-2}, \cdots, c_{1}$, segue que $c_{k}=0, \forall k=1, \cdots, N$. Logo, $\left\{f_{\alpha}: 0<\alpha<1\right\}$ é linearmente independente.

O próximo Lema apresenta uma ferramenta de cálculo que será utilizada na demonstração da Proposição 4.2.6.

Lema 4.2.5. Seja $f(z)=\frac{1}{z-3 / 2}$, então $\left(f_{\alpha}\right)^{(n)}=\alpha^{n}\left(f^{(n)}\right)_{\alpha}$.

Demonstração. A prova será por indução em $n$. Com efeito,

$$
\left(f_{\alpha}\right)^{\prime}(z)=(f(\alpha z))^{\prime}=\alpha f^{\prime}(\alpha z) \Rightarrow\left(f_{\alpha}\right)^{\prime}=\alpha\left(f^{\prime}\right)_{\alpha} .
$$

Dado $n \in \mathbb{N}$, suponha $\left(f_{\alpha}\right)^{(n)}=\alpha^{n}\left(f^{(n)}\right)_{\alpha}$. Como

$$
f^{(n)}=(-1)^{n} n ! f^{n+1} \text { e }\left(f^{n+1}\right)_{\alpha}^{\prime}=\alpha\left((n+1) f^{n} f^{\prime}\right)_{\alpha}=\alpha(n+1)(-1)\left(f^{n+2}\right)_{\alpha},
$$


temos

$$
\begin{aligned}
\left(f_{\alpha}\right)^{(n+1)} & =\left(\left(f_{\alpha}\right)^{(n)}\right)^{\prime} \\
& =\left(\alpha^{n}\left(f^{(n)}\right)_{\alpha}\right)^{\prime} \\
& =\alpha^{n}\left((-1)^{n} n ! f^{n+1}\right)_{\alpha}^{\prime} \\
& =\alpha^{n}(-1)^{n} n !\left(f^{n+1}\right)_{\alpha}^{\prime} \\
& =\alpha^{n+1}\left((-1)^{n+1}(n+1) ! f^{n+2}\right)_{\alpha} \\
& =\alpha^{n+1}\left(f^{(n+1)}\right)_{\alpha} .
\end{aligned}
$$

Proposição 4.2.6. (Lourenço - Vieira, [26]) Seja $f(z)=\frac{1}{z-3 / 2}$. Então, $\left[f_{\alpha}: 3 / 4<\alpha<1\right] \subset$ $\mathcal{H} \cup\{0\}$.

Demonstração. Uma vez que $f$ é uma função racional com polo fora de $\overline{\mathcal{D}}$, segue que $f \in \mathcal{A}(\mathcal{D})$. Vejamos agora que $f \in \mathcal{H}$. Com efeito, a partir do Lema 4.2.5, temos:

$$
\left\|\left(f_{\alpha}\right)^{(n)}\right\|_{\overline{\mathcal{D}}}=\alpha^{n}\left\|\left(f^{(n)}\right)_{\alpha}\right\|_{\overline{\mathcal{D}}}=\alpha^{n} n !\left\|f_{\alpha}\right\|_{\overline{\mathcal{D}}}^{n+1}=\alpha^{n} n !\left(\frac{2}{3-2 \alpha}\right)^{n+1}=n ! \frac{2}{3-2 \alpha}\left(\frac{2 \alpha}{3-2 \alpha}\right)^{n},
$$

pois $\sup _{|z| \leq 1}\left|\frac{1}{\alpha z-3 / 2}\right|=\frac{1}{|\alpha-3 / 2|}=\frac{2}{3-2 \alpha}$.

Como $\alpha>3 / 4$, temos $3-2 \alpha<3-2(3 / 4)=3-3 / 2=3 / 2$. Daí,

$$
\frac{2 \alpha}{3-2 \alpha}>\frac{3 / 2}{2 / 3}=9 / 4>1, \text { o que implica que }\left(\frac{2 \alpha}{3-2 \alpha}\right)^{n} \rightarrow+\infty \text {, quando } n \rightarrow \infty \text {. }
$$

Logo, $\sum_{n=0}^{\infty} \frac{1}{n !}\left\|f_{\alpha}^{(n)}\right\|_{\overline{\mathcal{D}}}=+\infty$, i.e $f_{\alpha} \in \mathcal{H}$.

Seja $g \in\left[f_{\alpha}: 3 / 4<\alpha<1\right]$. Vamos mostrar que se $g \neq 0$, então $g \in \mathcal{H}$. De fato, se $g \neq 0$, sejam $\beta_{1}, \cdots, \beta_{k} \in \mathbb{C} \backslash\{0\}$ e $3 / 4<\alpha_{1}, \cdots, \alpha_{k}<1$ tais que $g=\sum_{j=1}^{k} \beta_{j} f_{\alpha_{j}}$. Daí, pelo Lema 4.2.5,

$$
g^{(n)}(z)=\sum_{j=1}^{k} \beta_{j} f_{\alpha_{j}}^{(n)}(z)=\sum_{j=1}^{k} \beta_{j} \alpha_{j}^{n} n !(-1)^{n}\left(\frac{1}{\alpha_{j} z-3 / 2}\right)^{n+1} .
$$

Para concluir que $g \in \mathcal{H}$, é suficiente mostrar que $\lim _{n \rightarrow \infty}\left|\frac{g^{(n)}(1)}{n !}\right|=+\infty$, pois

$$
\frac{\left|g^{(n)}(1)\right|}{n !} \rightarrow+\infty \Rightarrow \sum_{n=0}^{\infty} \frac{1}{n !}\left\|g^{(n)}\right\|_{\mathcal{D}} \geq \sum_{n=0}^{\infty} \frac{1}{n !}\left|g^{(n)}(1)\right|=+\infty .
$$

Sendo assim, escreva

$$
\frac{g^{(n)}(1)}{n !}=\sum_{j=1}^{k}(-1)^{n} D_{j}\left(C_{j}\right)^{n}, \text { onde } C_{j}=\frac{2 \alpha_{j}}{2 \alpha_{j}-3} \text { e } D_{j}=\frac{2 \beta_{j}}{2 \alpha_{j}-3} .
$$

Sem perda de generalidade, suponha $C_{1}>C_{2}>\cdots C_{k}$, daí

$$
\frac{g^{(n)}(1)}{n !}=\left(C_{1}\right)^{n}(-1)^{n}\left(D_{1}+D_{2}\left(\frac{C_{2}}{C_{1}}\right)^{n}+D_{3}\left(\frac{C_{3}}{C_{1}}\right)^{n}+\cdots+D_{k}\left(\frac{C_{k}}{C_{1}}\right)^{n}\right) .
$$


Uma vez que $C_{1}>C_{j}, j=2, \cdots k$, temos

$$
\lim _{n \rightarrow \infty}\left(D_{1}+D_{2}\left(\frac{C_{2}}{C_{1}}\right)^{n}+D_{3}\left(\frac{C_{3}}{C_{1}}\right)^{n}+\cdots+D_{k}\left(\frac{C_{k}}{C_{1}}\right)^{n}\right)=D_{1} .
$$

Então, como $\left|C_{1}\right|>1$, segue que

$$
\lim _{n \rightarrow \infty}\left|\left(C_{1}\right)^{n}(-1)^{n}\right|=+\infty \text {, implicando que } \lim _{n \rightarrow \infty}\left|\frac{g^{(n)}(1)}{n !}\right|=+\infty .
$$

A partir do Lema 4.2.4 e da Proposição 4.2.6, Lourenço e Vieira mostraram que $\mathcal{H}$ é c-lineável.

Corolário 4.2.7. (Lourenço - Vieira, [26]) H é c-lineável.

Demonstração. Como $f(z)=\frac{1}{z-3 / 2} \in \mathcal{A}(\mathcal{D})$ não é um polinômio, pelo Lema 4.2.4, o conjunto $\left\{f_{\alpha}: 0<\alpha<1\right\}$ é linearmente independente. Assim, o conjunto $\left\{f_{\alpha}: 3 / 4<\alpha<1\right\}$ é base de $V=\left[f_{\alpha}: 3 / 4<\alpha<1\right]$ que está contido em $\mathcal{H} \cup\{0\}$ pela Proposição 4.2.6. Mas como $\operatorname{dim} V=$ card $((3 / 4,1))=$ card $(\mathbb{R})=\mathfrak{c}$, concluímos que $\mathcal{H}$ é $\mathfrak{c}$-lineável.

Vimos em 4.2.2 e em 4.2.7 que o conjunto $\mathcal{H}$ contém espaços vetoriais de dimensões infinitas. Como aplicação do Teorema 4.1.5, podemos mostrar que o conjunto $\mathcal{H}$ é espaçável. Ainda que o conceito de espaçabilidade implique lineabilidade. Apresentamos aqui todas as demonstrações, uma vez que elas apresentam técnicas diferentes.

Corolário 4.2.8. H é espaçável.

Demonstração. Sejam $M=(n !)_{n \in \mathbb{N}_{0}}$ e $D=D(\overline{\mathcal{D}}, M)$. A inclusão $i: D \rightarrow \mathcal{A}(\mathcal{D})$ é um operador linear contínuo entre os espaços de Banach, pois

$$
\|i(f)\|_{\overline{\mathcal{D}}}=\|f\|_{\overline{\mathcal{D}}} \leq \sum_{n=0}^{\infty} \frac{\left\|f^{(n)}\right\|_{\overline{\mathcal{D}}}}{n !}=\|f\|, \forall f \in D .
$$

Por outro lado, imagem $i(D)=D$ não é fechada em $\mathcal{A}(\mathcal{D})$. De fato, se $f=\frac{1}{z+2}$, pelo Lema 4.2.1, $f \in \mathcal{H}$. Mas como $f \in \mathcal{A}(\mathcal{D})$, pelo Teorema 2.1.4, existe sequência de polinômios $\left(p_{n}\right)$ tal que $\left\|p_{n}-f\right\|_{\overline{\mathcal{D}}} \rightarrow 0$. Em suma, $\left(p_{n}\right)$ é sequência em $D$ que converge para $f \notin D$. Assim, pelo Teorema 4.1.5, concluímos que $\mathcal{A}(\mathcal{D}) \backslash i(D)=\mathcal{A}(\mathcal{D}) \backslash D=\mathcal{H}$ é espaçável.

Note que se $M_{n} \leq n$ ! e $f \in \mathcal{H}$, então

$$
\sum_{n=0}^{\infty} \frac{1}{M_{n}}\|f\|_{\overline{\mathcal{D}}} \geq \sum_{n=0}^{\infty} \frac{1}{n !}\|f\|_{\overline{\mathcal{D}}}=+\infty .
$$

Portanto, temos $\mathcal{H} \subset \mathcal{H}(M)$. Assim, os espaços vetoriais de 4.2.2, 4.2.7 e 4.2.8 também estão contidos em $\mathcal{H}(M) \cup\{0\}$. Desse modo, temos o seguinte resultado:

Corolário 4.2.9. Se $M=\left(M_{n}\right)$ é uma sequência de álgebra tal que $M_{n} \leq n$ !, para todo $n \in \mathbb{N}$. Então, $\mathcal{H}(M)$ é $\aleph_{0}$-lineável, $\mathfrak{c}$-lineável e espaçável.

As sequência de álgebras apresentadas no item 1 de 3.1 .2 são tais que $M_{n} \leq n !, \forall n \in \mathbb{N}$. 


\subsection{Algebrabilidade}

Nessa seção, vamos estudar a algebrabilidade do conjunto $\mathcal{H}$. Os resultados desta seção podem ser encontrados em [25, 26]. Em [25], Lourenço e Vieira mostraram que o conjunto é algebrável. Enquanto em [26], aplicaram o Teorema 4.1.6 para mostrar que $\mathcal{H}$ é fortemente $\mathfrak{c}$-algebrável.

O próximo resultado foi provado por Lourença e Vieira em [25]. Lourenço e Vieira utilizam o Lema nas demonstrações dos Teoremas 4.3.2 e 4.3.4.

Lema 4.3.1. (Lourenço - Vieira, [25]) Sejam $\beta>0$ e $n \in \mathbb{N}$. Para cada $r=1, \cdots, n$, considere

$$
a_{r}=\frac{1}{r !} \sum_{s=1}^{r}\left(\begin{array}{l}
r \\
s
\end{array}\right)(-1)^{r-s+n} s(s+1) \cdots(s+n-1) .
$$

Então,

1. Se $K \in \mathbb{N}$ é tal que $1 \leq r \leq K$, e n é par, temos $\lim _{n \rightarrow \infty} \beta^{r} \frac{a_{r}}{n !}=+\infty$.

2. $\lim _{n \rightarrow \infty} \lim _{r \rightarrow n} \beta^{r} \frac{a_{r}}{n !}=0$.

Demonstração. Primeiramente, note que:

$$
\begin{aligned}
\frac{a_{r}}{n !} & =\frac{1}{n ! r !} \sum_{s=1}^{r}\left(\begin{array}{l}
r \\
s
\end{array}\right)(-1)^{r-s+n} s(s+1) \cdots(s+n-1) \\
& =\sum_{s=1}^{r} \frac{r !}{(r-s) ! s !}(-1)^{r-s+n} \frac{s(s+1) \cdots(s+n-1)}{n ! r !} \\
& =\sum_{s=1}^{r}(-1)^{r-s+n} \frac{(s+n-1) !}{(s-1) !(r-s) ! s ! n !} \\
& =\sum_{s=1}^{r}(-1)^{r-s+n} \frac{(n+1)(n+2) \cdots(s+n-1)}{(s-1) !(r-s) ! s !} .
\end{aligned}
$$

Desse modo, cada termo da soma acima é um polinômio em $n$ com grau $s-1$, $\operatorname{logo} a_{r} / n$ ! é um polinômio em $n$ com grau $r-1$. Seja, agora, $K$ um inteiro positivo tal que $1 \leq r \leq K$. Assim, $\beta^{r} \leq \beta^{K}$ é limitado. Além disso, se $n$ é par, o termo de maior grau de $a_{r} / n$ ! é

$$
(-1)^{n} \frac{(n+1) \cdots(n+r-1)}{r !(r-1) !}=\frac{(n+1) \cdots(n+r-1)}{r !(r-1) !}>0 .
$$

Então,

$$
\lim _{n \rightarrow \infty} \frac{a_{r}}{n !}=+\infty \Rightarrow \lim _{n \rightarrow \infty} \beta^{r} \frac{a_{r}}{n !}=+\infty
$$

Para provar o item 2, observe que:

$$
\begin{aligned}
\lim _{r \rightarrow n} \lim _{s \rightarrow r} \beta^{r}(-1)^{r-s+n} \frac{(s+n-1) !}{(r-s) ! s !(s-1) ! n !} & =\lim _{r \rightarrow n} \beta^{r}(-1)^{n} \frac{(r+n-1) !}{r !(r-1) ! n !} \\
& =\beta^{n}(-1)^{n} \frac{(2 n-1) !}{n ! n !(n-1) !} .
\end{aligned}
$$


Se $b_{n}=\beta^{n}(-1)^{n} \frac{(2 n-1) !}{n ! n !(n-1) !}, \forall n$, temos:

$$
\begin{aligned}
\frac{b_{n+1}}{b_{n}} & =-\beta \frac{(2(n+1)-1) !}{(n+1) !(n+1) ! n !} \frac{n ! n !(n-1) !}{(2 n-1) !} \\
& =-\beta \frac{(2 n+1) ! n !(n-1) !}{(n+1) !(n+1) !(2 n-1) !} \\
& =-\beta \frac{(2 n+1)(2 n)}{(n+1)(n+1) n} \\
& =-\beta \frac{4 n+2}{n^{2}+2 n+1} \\
& =-\beta \frac{n(4+2 / n)}{n^{2}\left(1+2 / n+1 / n^{2}\right)} \\
& =-\frac{\beta}{n} \frac{4+2 / n}{1+2 / n+1 / n^{2}} \\
& \stackrel{n \rightarrow \infty}{\rightarrow} 0 .
\end{aligned}
$$

Assim, pelo critério de D'Alembert, segue que $\sum b_{n}$ converge absolutamente e portanto $b_{n} \rightarrow 0$. Daí,

$$
\lim _{n \rightarrow \infty} \lim _{r \rightarrow n} \beta^{r} \frac{a_{r}}{n !}=0 .
$$

Em [25], foi apresentada uma certa classe de funções contidas em $\mathcal{H} \cup\{0\}$ como veremos no próximo resultado.

Teorema 4.3.2. (Lourenço - Vieira, [25]) Sejam $g_{1}(z)=e^{z}$ e $g_{2}(z)=e^{\beta z}, \forall z \in \mathbb{C}$, onde $\beta \in \mathbb{R} \backslash \mathbb{Q}$. Sejam $P \in \mathbb{C}\left[z_{1}, z_{2}\right]$ um polinômio não constante, $f(z)=\frac{1}{z+2} e h=P\left(g_{1}, g_{2}\right)$. Então, $h \circ f \in \mathcal{H} \cup\{0\}$.

Demonstração. Pelo Lema 4.2.1, $f \in \mathcal{H}$. Além disso, pelo Princípio do Módulo Máximo - 2.1.3,

$$
\|f\|_{\overline{\mathcal{D}}}=\sup _{z \in \overline{\mathcal{D}}}|f(z)|=\max _{z \in \partial \overline{\mathcal{D}}} \frac{1}{|z+2|}=\min _{z \in \partial \overline{\mathcal{D}}}|z+2|=1 .
$$

Seja $P\left(z_{1}, z_{2}\right)=\sum_{j, k=0}^{m} \alpha_{j k} z_{1}^{j} z_{2}^{k}$ um polinômio não constante. Assim,

$$
h(z)=P\left(g_{1}, g_{2}\right)=\sum_{j, k=0}^{m} \alpha_{j k} e^{(j+\beta k) z}=\sum_{j, k=0}^{m} \alpha_{j k} e^{\beta_{j k} z}, \forall z \in \mathbb{C},
$$

onde $\beta_{j k}=j+\beta k$. Pela Fórmula de Hoppe (Proposição 1.1.2),

$$
(h \circ f)^{(n)}=\sum_{r=1}^{n} \frac{h^{(r)}(f(z))}{r !} \sum_{s=0}^{r}\left(\begin{array}{l}
r \\
s
\end{array}\right)(-f(z))^{r-s}\left(f^{s}\right)^{(n)}(z) .
$$

$\operatorname{Mas}$ como $\left(f^{s}\right)^{(n)}=(-1)^{n} s(s+1) \cdots(s+n-1) f^{s+n}, f(-1)=1$ e $h^{(r)}(1)=\sum_{j, k=0}^{m} \alpha_{j k} \beta_{j k}^{r} e^{\beta_{j k}}$ temos:

$$
(h \circ f)^{(n)}(-1)=\sum_{r=1}^{n} \frac{1}{r !}\left(\sum_{j, k=0}^{m} \alpha_{j k} \beta_{j k}^{r} e^{\beta_{j k}}\right) \sum_{s=0}^{r}\left(\begin{array}{l}
r \\
s
\end{array}\right)(-1)^{r-s+n} s(s+1) \cdots(s+n-1) .
$$

Desse modo, com a notação do Lema 4.3.1,

$$
(h \circ f)^{(n)}(-1)=\sum_{r=1}^{n} \sum_{j, k=0}^{m} \alpha_{j k} \beta_{j k}^{r} e^{\beta_{j k}} a_{r} .
$$


Vamos provar que $\sum_{n=0}^{\infty} \frac{\left|(h \circ f)^{(n)}(-1)\right|}{n !}=+\infty$. Em caso afirmativo, teremos

$$
\sum_{n=0}^{\infty} \frac{\left\|(h \circ f)^{(n)}\right\|_{\overline{\mathcal{D}}}}{n !} \geq \sum_{n=0}^{\infty} \frac{\left|(h \circ f)^{(n)}(-1)\right|}{n !}=+\infty, \text { o que implica que } h \circ f \in \mathcal{H} \cup\{0\} .
$$

Com efeito, para cada $n \in \mathbb{N}_{0}$, sejam

$$
H_{n}=\frac{(h \circ f)^{(n)}(-1)}{n !} \text { e } L_{r}=\sum_{j, k=0}^{m} \alpha_{j k} \beta_{j k}^{r} e^{\beta_{j k}}
$$

Assim,

$$
H_{n}=\sum_{r=1}^{n} \frac{a_{r}}{n !} L_{r}
$$

Veremos que $L_{N_{0}} \neq 0$ para algum $N_{0}$. Para melhor compreensão, ilustraremos aqui o caso em que $m=2$. Se, por contradição, $L_{r}=0, \forall r$, como $\beta_{00}=0$, temos o seguinte sistema:

$$
\left[\begin{array}{cccc}
\beta_{01} e^{\beta_{01}} & \beta_{02} e^{\beta_{02}} & \cdots & \beta_{22} e^{\beta_{22}} \\
\beta_{01}^{2} e^{\beta_{01}} & \beta_{02}^{2} e^{\beta_{02}} & \cdots & \beta_{22}^{2} e^{\beta_{22}} \\
\vdots & \vdots & \ddots & \vdots \\
\beta_{01}^{8} e^{\beta_{01}} & \beta_{02}^{8} e^{\beta_{02}} & \cdots & \beta_{22}^{8} e^{\beta_{22}}
\end{array}\right]\left[\begin{array}{c}
\alpha_{01} \\
\alpha_{02} \\
\vdots \\
\alpha_{22}
\end{array}\right]=\left[\begin{array}{c}
0 \\
0 \\
\vdots \\
0
\end{array}\right] .
$$

Pelo mesmo argumento utilizado no Exemplo 4.1.3, concluímos que $\alpha_{i j}=0, \forall(i, j) \neq(0,0)$. Então, $P=\alpha_{00}$ é constante, uma contradição. Para o caso com $m$ arbitrário, teríamos um sistema com $(m+1)^{2}-1$ variáveis e obteríamos a mesma conclusão.

Portanto, existe $N_{0} \in \mathbb{N}$ tal que $L_{N_{0}} \neq 0$, i.e

$$
H_{n}=\frac{a_{1}}{n !} L_{1}+\cdots+\frac{a_{N_{0}}}{n !} L_{N_{0}}+\cdots+\frac{a_{n}}{n !} L_{n}, \forall n>N_{0} .
$$

Dado $n>N_{0}$ par, temos:

$$
\lim _{n \rightarrow \infty} \beta_{j k}^{r} \frac{a_{r}}{n !}=+\infty, \forall r=1, \cdots, N_{0}, L_{r} \neq 0 \quad \text { e } \quad \lim _{n \rightarrow \infty} \lim _{r \rightarrow n} \beta_{j k}^{r} \frac{a_{r}}{n !}=0, \forall r>N_{0} .
$$

Por conseguinte,

$$
\lim _{n \rightarrow \infty}\left|H_{n}\right|=\left|\sum_{j, k=0}^{m} \alpha_{j k} e^{\beta_{j k}} \lim _{n \rightarrow \infty}\left(\beta_{j k}^{r} \frac{a_{r}}{n !}\right)\right|=+\infty \text {, implicando que } \sum\left|H_{n}\right|=+\infty .
$$

Agora, apresentaremos a demonstração de que $\mathcal{H}$ é um conjunto algebrável.

Teorema 4.3.3. (Lourenço - Vieira, [25]) H é algebrável.

Demonstração. Sejam $g_{1}(z)=e^{z}$ e $g_{2}(z)=e^{\beta z}$, onde $\beta \in \mathbb{R} \backslash \mathbb{Q}$. Vimos no Exemplo 4.1.3 que $g_{1}$ e $g_{2}$ são algebricamente independentes, assim $\mathcal{A}\left(\left\{g_{1}, g_{2}\right\}\right)$ e $\mathbb{C}\left[z_{1}, z_{2}\right]$ são álgebras isomorfas. Considere o conjunto

$$
S=\left\{g_{1} g_{2}^{n}: n \in \mathbb{N}_{0}\right\} .
$$

É imediato que $\mathcal{B}=\mathcal{A}(S) \subset \mathcal{A}\left(\left\{g_{1}, g_{2}\right\}\right)$. Vamos provar que $S$ é um sistema minimal de geradores para $\mathcal{B}$. Com efeito, dado $i_{0} \in \mathbb{N}_{0}$, veremos que $g_{1} g_{2}^{i_{0}} \notin \mathcal{A}\left(S \backslash\left\{g_{i_{0}}\right\}\right)$. Caso $\alpha_{i_{0}} g_{1} g_{2}^{i_{0}}+$ 
$\sum_{j=1}^{k} \alpha_{j}\left(g_{1} g_{2}^{m}\right)^{j}=0$, com $m \neq i_{0}$, i.e.

$$
\alpha_{i_{0}} e^{\left(1+\beta i_{0}\right) z}+\sum_{j=1}^{k} \alpha_{j} e^{(1+\beta(m j)) z}=0, \text { consequentemente } \alpha_{i_{0}} e^{\beta_{i_{0}} z}+\sum_{j=1}^{k} \alpha_{j} e^{\beta_{j} z}=0,
$$

onde $\beta_{i_{0}}=1+\beta i_{0}$ e $\beta_{j}=1+\beta(m j)$. Derivando a equação $r$ vezes e aplicando em $z=1$, temos:

$$
\alpha_{i_{0}} \beta_{i_{0}}^{r} e^{\beta_{i_{0}} z}+\sum_{j=1}^{k} \alpha_{j} \beta_{j}^{r} e^{\beta_{j} z}=0, \forall r
$$

Em notação matricial:

$$
\left[\begin{array}{cccc}
\beta_{i_{0}} e^{\beta_{i_{0}}} & \beta_{1} e^{\beta_{1}} & \cdots & \beta_{k} e^{\beta_{k}} \\
\beta_{i_{0}}^{2} e^{\beta_{i_{0}}} & \beta_{1}^{2} e^{\beta_{1}} & \cdots & \beta_{k}^{2} e^{\beta_{k}} \\
\vdots & \vdots & \ddots & \vdots \\
\beta_{i_{0}}^{k+1} e^{\beta_{i_{0}}} & \beta_{1}^{k+1} e^{\beta_{1}} & \cdots & \beta_{k}^{k+1} e^{\beta_{k}}
\end{array}\right]\left[\begin{array}{c}
\alpha_{i_{0}} \\
\alpha_{1} \\
\vdots \\
\alpha_{k}
\end{array}\right]=\left[\begin{array}{c}
0 \\
0 \\
\vdots \\
0
\end{array}\right]
$$

Com o mesmo argumento utilizado no Exemplo 4.1.3, segue que $\alpha_{i_{0}}=\alpha_{1}=\cdots=\alpha_{k}=0$.

Sejam $f(z)=1 /(z+2)$ e $W=\left\{\left(g_{1} g_{2}^{n}\right) \circ f: n \in \mathbb{N}_{0}\right\}$. Então, como $S$ é minimal, $W$ também o é. Além disso, $\mathcal{F}=\{h \circ f: h \in \mathcal{B}\}=\mathcal{A}(W)$. De fato, se $g \in \mathcal{F}$, existe $h \in \mathcal{B}, g=h \circ f$. Mas como $h=\sum_{i=1}^{n} \alpha_{i}\left(g_{1} g_{2}^{j}\right)^{i}$ para algum $j \in \mathbb{N}$, temos

$$
g=\sum_{i=1}^{n} \alpha_{i}\left[\left(g_{1} g_{2}^{j}\right)^{i} \circ f\right]=\sum_{i=1}^{n} \alpha_{i}\left[\left(g_{1} g_{2}^{j}\right) \circ f\right]^{i} \in \mathcal{A}(W) .
$$

Portanto, $\mathcal{F} \subset \mathcal{A}(W)$. Por outro lado, se $g \in \mathcal{A}(W)$, segue que

$$
g=\sum_{i=1}^{n} \alpha_{i}\left[\left(g_{1} g_{2}^{j}\right) \circ f\right]^{i}=\sum_{i=1}^{n} \alpha_{i}\left[\left(g_{1} g_{2}^{j}\right)^{i} \circ f\right]=\left[\sum_{i=1}^{n} \alpha_{i}\left(g_{1} g_{2}^{j}\right)^{i}\right] \circ f \in \mathcal{F},
$$

concluindo que $\mathcal{A}(W) \subset \mathcal{F}$.

Assim, pelo Teorema 4.3.2, dado $h \in \mathcal{A}\left(\left\{g_{1}, g_{2}\right\}\right) \backslash\{0\}, h \circ f \in \mathcal{H}$, pois $h=P\left(g_{1}, g_{2}\right)$ e $P \in$ $\mathbb{C}\left[z_{1}, z_{2}\right]$ é não constante. Caso, $h=0$, temos $h \circ f=0$. Então, $\mathcal{F} \subset \mathcal{H} \cup\{0\}$. Em suma, $\mathcal{F}$ é uma subálgebra de $\mathcal{A}(\mathcal{D})$ gerada por um sistema minimal de geradores infinito que está contida em $\mathcal{H} \cup\{0\}$.

Em [26], Lourenço e Vieira mostraram, a partir do Teorema 4.1.6, que $\mathcal{H}$ é fortemente $\mathfrak{c}$ algebrável, melhorando, pois, o Teorema 4.3.3, obtido em [25].

Teorema 4.3.4. (Lourenço - Vieira, [26]) H é fortemente c-algebrável.

Demonstração. Seja $f(z)=\frac{1}{z+2}$. Pelo Lema 4.2.1, $f \in \mathcal{H}$. Se $\varphi \in \mathcal{E}$, existem $\alpha_{1}, \cdots, \alpha_{m} \in \mathbb{C} \backslash\{0\}$ e existem $b_{1}, \cdots, b_{m} \in \mathbb{C} \backslash\{0\}$ dois a dois distintos tais que $\varphi(z)=\sum_{j=1}^{m} \alpha_{j} e^{b_{j} z}, \forall z \in \mathbb{C}$. Desse modo,

$$
(\varphi \circ f)(z)=\sum_{j=1}^{m} \alpha_{j} e^{b_{j} f(z)} \in \mathcal{A}(\mathcal{D}) .
$$

Aplicando a Fórmula de Hoppe e prosseguindo de modo análogo à demonstração do Teorema 4.3.3, segue que

$$
(\varphi \circ f)^{(n)}(-1)=\sum_{r=1}^{n} \sum_{j=1}^{m} \alpha_{j} b_{j}^{r} e^{b_{j}} a_{r}
$$


onde

$$
a_{r}=\frac{1}{r !} \sum_{s=0}^{r}\left(\begin{array}{l}
r \\
s
\end{array}\right)(-1)^{r-s+n} s(s+1) \cdots(s+n-1) .
$$

Para cada $n$ e $r=1, \cdots, n$, sejam

$$
H_{n}=\frac{(\varphi \circ f)^{(n)}(-1)}{n !} \text { e } L_{r}=\sum_{j=1}^{m} \alpha_{j} b_{j}^{r} e^{b_{j}} .
$$

Isto é, $H_{n}=\sum_{r=1}^{n} \frac{a_{r}}{n !} L_{r}$

Suponha, por contradição, que $L_{r}=0, \forall r$. Assim, temos o seguinte sistema matricial:

$$
\left[\begin{array}{cccc}
b_{1} e^{b_{1}} & b_{2} e^{b_{2}} & \cdots & b_{m} e^{b_{m}} \\
b_{1}^{2} e^{b_{1}} & b_{2}^{2} e^{b_{2}} & \cdots & b_{m}^{2} e^{b_{m}} \\
\vdots & \vdots & \ddots & \vdots \\
b_{1}^{m} e^{b_{1}} & b_{2}^{m} e^{b_{2}} & \cdots & b_{m}^{m} e^{b_{m}}
\end{array}\right]\left[\begin{array}{c}
\alpha_{1} \\
\alpha_{2} \\
\vdots \\
\alpha_{m}
\end{array}\right]=\left[\begin{array}{c}
0 \\
0 \\
\vdots \\
0
\end{array}\right]
$$

A matriz dos coeficientes do sistema acima é uma matriz de Vandermonde cujo determinante é

$$
b_{1} \cdots b_{m} e^{b_{1}} \cdots e^{b_{m}} \prod_{1 \leq i<j \leq m}\left(b_{i}-b_{j}\right) \neq 0
$$

pois os $b_{j}^{\prime} s$ são não nulos e dois a dois distintos. Daí, teríamos $\alpha_{1}=\cdots=\alpha_{r}=0$, uma contradição.

Portanto, existe $N_{0} \in\{1, \cdots, m\}$ tal que $L_{N_{0}} \neq 0$, logo

$$
H_{n}=\frac{a_{1}}{n !} L_{1}+\cdots+\frac{a_{N_{0}}}{n !} L_{N_{0}}+\cdots+\frac{a_{n}}{n !} L_{n}, \forall n>N_{0} .
$$

Aplicando o Lema 4.3.1, se $n>N_{0}$ par, temos:

$$
\lim _{n \rightarrow \infty} b_{j}^{r} \frac{a_{r}}{n !}=+\infty, \forall r=1, \cdots, N_{0}, L_{r} \neq 0 \quad \text { e } \quad \lim _{n \rightarrow \infty} \lim _{r \rightarrow n} b_{j}^{r} \frac{a_{r}}{n !}=0, \forall r>N_{0} .
$$

Por conseguinte,

$$
\lim _{n \rightarrow \infty}\left|H_{n}\right|=\left|\sum_{j=1}^{m} \alpha_{j} e^{b_{j}} \lim _{n \rightarrow \infty}\left(b_{j}^{r} \frac{a_{r}}{n !}\right)\right|=+\infty .
$$

Logo, $\sum\left|H_{n}\right|=+\infty$ e, portanto, $\varphi \circ f \in \mathcal{H}$. Por outro lado, se $z_{1}, z_{2} \in \overline{\mathcal{D}}$ com $z_{1} \neq z_{2}$, então $z_{1}+2 \neq z_{2}+2$, o que implica que $f\left(z_{1}\right) \neq f\left(z_{2}\right)$, e portanto, $f$ é injetora em $\overline{\mathcal{D}}$. Daí, como $\overline{\mathcal{D}}$ é não enumerável, $f(\overline{\mathcal{D}})$ também é não enumerável.

Em suma, $\mathcal{H}$ é uma família de funções de $\overline{\mathcal{D}} \rightarrow \mathbb{C}$ e $f \in \mathcal{H}$ é tal que $f(\overline{\mathcal{D}})$ é não-enumerável e $\varphi \circ f \in \mathcal{H}, \forall \varphi \in \mathcal{E}$. Logo, pelo Teorema 4.1.6, $\mathcal{H}$ é fortemente $\mathfrak{c}$-algebrável.

Vimos na seção anterior que se $M_{n} \leq n$ !, temos $\mathcal{H} \subset \mathcal{H}(M)$. Desse modo, a as álgebras de 4.3.3 e 4.3.4 também estão contidas em $\mathcal{H}(M)$. Obtémos, pois, o seguinte Corolário:

Corolário 4.3.5. (Lourenço - Vieira, [26]) Seja $\left(M_{n}\right)$ sequência de álgebra tal que $M_{n} \leq n !, \forall n \in$ $\mathbb{N}$. Então, $\mathcal{H}(M)$ é algebrável e fortemente $\mathfrak{c}$-algebrável.

\subsection{Residualidade}

Para concluir este capítulo, apresentaremos a demonstração de que $\mathcal{H}$ é residual em $\mathcal{A}(\mathcal{D})$. Recordemos que $f \in D(\overline{\mathcal{D}}, M)$ se, e só se, $f \in D^{\infty}(\overline{\mathcal{D}})$ e $\sum_{k=0}^{\infty} \frac{\left\|f^{(k)}\right\|_{\overline{\mathcal{D}}}}{k !}<\infty$. 
Nos resultados provados anteriormente, não importava se $f \in D^{\infty}(\overline{\mathcal{D}})$ ou $f \notin \mathcal{A}(\mathcal{D})$. Não obstante, as funções utilizadas para encontrar subespaços e subálgebras eram da forma $f(z)=$ $\frac{1}{z-2 e^{i \theta}} \in D^{\infty}(\overline{\mathcal{D}})$.

Para mostrar a residualidade, precisamos fazer a seguinte distinção:

$$
f \in \mathcal{H} \Longleftrightarrow f \in D^{\infty}(X) \text { e } \sum_{k=0}^{\infty} \frac{\left\|f^{(k)}\right\|_{X}}{k !}=+\infty .
$$

No que segue vamos considerar

$$
\mathcal{H}=\left\{f \in D^{\infty}(\overline{\mathcal{D}}): \sum_{k=0}^{\infty} \frac{\left\|f^{(k)}\right\|_{X}}{k !}=+\infty\right\} \subset \mathcal{A}(\mathcal{D}) .
$$

A Estimativa de Cauchy é um conhecido resultado do tópico de análise complexa cuja demonstração pode ser encontrada em livros básicos, e.g. [10]. Vamos utilizar este resultado para demonstrarmos o Lema 4.4.3.

Lema 4.4.1. (Estimativa de Cauchy) Sejam $z_{0} \in \mathbb{C}, R>0$ e $f$ uma função holomorfa em $B\left(z_{0}, R\right)$. Suponha que $|f(z)| \leq L, \forall z \in B\left(z_{0}, R\right)$, então para cada $k \in \mathbb{N}$,

$$
\left|f^{(k)}\right| \leq \frac{k ! L}{R^{k}}
$$

Segue do Princípio do Módulo Máximo que se $f \in D^{\infty}(\overline{\mathcal{D}})$ é tal que $\|f\|_{\overline{\mathcal{D}}}>L$, então existe um ponto $\bar{z} \in \partial \overline{\mathcal{D}}$ tal que $\|f\|_{\overline{\mathcal{D}}}=|f(\bar{z})|>L$. No próximo resultado, vamos demonstrar que podemos encontrar $z_{0} \in \mathcal{D}$ tal que $\|f\|_{\overline{\mathcal{D}}} \geq\left|f\left(z_{0}\right)\right|>L$.

Lema 4.4.2. Seja $f \in D^{\infty}(\overline{\mathcal{D}})$ tal que $\|f\|_{\overline{\mathcal{D}}}>$ L. Então, existe $z_{0} \in \mathcal{D}$ tal que $\left|f\left(z_{0}\right)\right|>L$.

Demonstração. Em particular, $f$ é uma função analítica em $\mathcal{D}$, então existe $z^{\prime} \in \partial \mathcal{D}$ tal que $\left|f\left(z^{\prime}\right)\right|=$ $\|f\|_{\overline{\mathcal{D}}}>L$. Por outro lado, temos que a função $h:[0,1] \rightarrow \mathbb{R}$ definida por $h(t)=\left|f\left(t z^{\prime}\right)\right|$ é uma função contínua tal que $h(1)=\left|f\left(z^{\prime}\right)\right|$ é o seu valor máximo e $h(1)>L$. Vamos dividir a demonstração em três casos.

Caso $h(t)>L, \forall t$, temos, em particular, para $t_{0}=1 / 2$ que $z_{0}=\frac{1}{2} z^{\prime} \in D$ e $\left|f\left(z_{0}\right)\right|>L$.

Caso exista $t_{1}$ com $h\left(t_{1}\right)=L$. Como $L<h(1)$, existe $d \in \mathbb{R}$ tal que $h\left(t_{1}\right)<d<h(1)$. Assim, pelo Teorema do Valor Intermediário, existe $t_{0} \in\left(t_{1}, 1\right)$ tal que $h\left(t_{0}\right)=d$. Isto é, se $z_{0}=t_{0} z^{\prime} \in \mathcal{D}$, $\left|f\left(z_{0}\right)\right|>L$.

Caso exista $t_{1}$ com $h\left(t_{1}\right)<L$. Como $L<h(1)$, pelo Teorema do Valor Intermediário, existe $t_{2} \in\left(t_{1}, 1\right)$ tal que $h\left(t_{2}\right)=L$. Desse modo, pelo caso anterior, existe $t_{0} \in\left(t_{2}, 1\right)$ tal que $\left|f\left(z_{0}\right)\right|=$ $h\left(t_{0}\right)>L$, onde $z_{0}=t_{0} z^{\prime} \in \mathcal{D}$.

Em suma, existe $z_{0} \in \mathcal{D}$ tal que $\left|f\left(z_{0}\right)\right|>L$.

Pela Observação 1.2.10, para concluir que $\mathcal{H}$ é residual em $\mathcal{A}(\mathcal{D})$ é suficiente mostrar que $\mathcal{H}$ se escreve como intersecção enumerável de abertos densos em $\mathcal{A}(\mathcal{D})$. No próximo Lema, vamos construir uma família de abertos em $\mathcal{A}(\mathcal{D})$.

Lema 4.4.3. Dados $n, m \in \mathbb{N}_{0}$, temos que o conjunto

$$
S_{m, n}=\left\{f \in D^{\infty}(\overline{\mathcal{D}}): \sum_{k=0}^{m} \frac{1}{k !}\left\|f^{(k)}\right\|_{\overline{\mathcal{D}}}>n\right\}
$$

é aberto em $\mathcal{A}(\mathcal{D})$.

Demonstração. Caso $n=0, f \in S_{m, 0} \Longleftrightarrow \sum_{k=0}^{m} \frac{1}{k !}\left\|f^{(k)}\right\|_{\overline{\mathcal{D}}}>0$. Assim, o complementar de $S_{m, 0}$ é o conjunto unitário $\{0\}$. Logo, $S_{m, 0}$ é um aberto. Caso $m=0, f \in S_{0, n} \Longleftrightarrow\|f\|_{\bar{D}}>n$. Logo, o complementar de $S_{0, n}$ é a bola fechada de centro na origem e raio $n$. Então, $S_{0, n}$ é aberto. 
Dados $m, n \in \mathbb{N}$, como o vazio é um conjunto aberto, vamos supor que $S_{m, n} \neq \emptyset$. Suponha, por absurdo, que $S_{m, n}$ não seja um conjunto aberto. Daí, existe $f \in S_{m, n}$ tal que para todo $\epsilon>0$, existe $g_{\epsilon} \in B(f, \epsilon)$ com $g \notin S_{m, n}$. Por um lado, $f \in S_{m, n}$, então $\sum_{k=0}^{m} \frac{1}{k !}\left\|f^{(k)}\right\|_{\overline{\mathcal{D}}}>n$. Em particular, $\|f\|_{\overline{\mathcal{D}}}>n-\sum_{k=1}^{m} \frac{1}{k !}\left\|f^{(k)}\right\|_{\overline{\mathcal{D}}}$. Pelo Lema 4.4.2, existe $z_{0} \in \mathcal{D},\left|f\left(z_{0}\right)\right|>n-\sum_{k=1}^{m} \frac{1}{k !}\left\|f^{(k)}\right\|_{\overline{\mathcal{D}}}$. Podemos repetir o argumento acima mais $m$ vezes de modo a encontrar $z_{1}, \cdots, z_{m} \in \mathcal{D}$ tais que $\sum_{k=0}^{m} \frac{1}{k !}\left|f^{(k)}\left(z_{k}\right)\right|>n$. Para cada $k=1, \cdots, m$, defina $r_{k}=\left(1-\left|r_{k}\right|\right)$.

Por outro lado, como $B\left(z_{k}, r_{k}\right) \subset D$, temos que $f-g_{\epsilon}$ é holomorfa em $B\left(z_{k}, r_{k}\right)$ e $\left|\left(f-g_{\epsilon}\right)(z)\right| \leq$ $\left\|f-g_{\epsilon}\right\|_{\overline{\mathcal{D}}}, \forall z \in B\left(z_{k}, r_{k}\right)$. Assim, pelo Lema 4.4.1:

$$
\frac{\left|f^{(k)}\left(z_{k}\right)-g_{\epsilon}^{(k)}\left(z_{k}\right)\right|}{k !} \leq \frac{\left\|f-g_{\epsilon}\right\|_{\overline{\mathcal{D}}}}{r_{k}^{k}}, \forall k=1, \cdots, m .
$$

Daí, pela desigualdade triangular e pelo fato que $g \notin S_{m, n}$, temos:

$$
\begin{aligned}
\sum_{k=0}^{m} \frac{\left|f^{(k)}\left(z_{k}\right)\right|}{k !} & \leq \sum_{k=0}^{m} \frac{\left|f^{(k)}\left(z_{k}\right)-g_{\epsilon}^{(k)}\left(z_{k}\right)\right|}{k !}+\sum_{k=0}^{m} \frac{\left|g_{\epsilon}^{(k)}\left(z_{k}\right)\right|}{k !} \\
& \leq\left\|f-g_{\epsilon}\right\|_{\overline{\mathcal{D}}}\left(1+1 / r_{1}+\left(1 / r_{2}\right)^{2} \cdots\left(1 / r_{m}\right)^{m}\right)+n \\
& \leq \epsilon\left(1+1 / r_{1}+\left(1 / r_{2}\right)^{2} \cdots\left(1 / r_{m}\right)^{m}\right)+n, \forall \epsilon>0 .
\end{aligned}
$$

Tomando $\epsilon \rightarrow 0$, teríamos $\sum_{k=0}^{m} \frac{\left|f^{(k)}\left(z_{k}\right)\right|}{k !} \leq n$, uma contradição. Portanto, $S_{m, n}$ é aberto.

Com o auxílio do Lema 4.4.3, podemos apresentar a demonstração de que $\mathcal{H}$ é residual.

Teorema 4.4.4. (Lourenço - Vieira, [26]) $\mathcal{H}$ é residual em $\mathcal{A}(\mathcal{D})$.

Demonstração. A partir da Observação 1.2.10, é suficiente provar que $\mathcal{H}$ pode ser escrito como interseção enumerável de abertos densos de $\mathcal{A}(\mathcal{D})$. Com efeito, dados $m, n \in \mathbb{N}$, defina

$$
S_{m, n}=\left\{f \in D^{\infty}(\overline{\mathcal{D}}): \sum_{k=0}^{m} \frac{1}{k !}\left\|f^{(k)}\right\|_{\overline{\mathcal{D}}}>n\right\} \text { e } S_{n}=\bigcup_{m \geq n} S_{m, n} .
$$

Pelo Lema 4.4.3, cada conjunto $S_{m, n} \subset D^{\infty}(\overline{\mathcal{D}}) \subset \mathcal{A}(\mathcal{D})$ é aberto em $\mathcal{A}(\mathcal{D})$. Logo, $S_{n}=\bigcup_{m \geq n} S_{m, n}$ é aberto em $\mathcal{A}(\mathcal{D})$, para todo $n$.

Veremos, agora, que $\mathcal{H}=\bigcap_{n \in \mathbb{N}} S_{n}$. Com efeito, se $f \in \mathcal{H}$, como $\sum_{k=0}^{\infty} \frac{1}{k !}\left\|f^{(k)}\right\|_{\overline{\mathcal{D}}}=+\infty$, dado $n \in \mathbb{N}$, existe $m \in \mathbb{N}$ tal que

$$
\sum_{k=0}^{m} \frac{1}{k !}\left\|f^{(k)}\right\|_{\overline{\mathcal{D}}}>n \text {, i.e } f \in S_{m, n} .
$$

Sem perda de generalidade, podemos supor $m \geq n$. Logo, $f \in S_{n}, \forall n \in \mathbb{N}$ e, por conseguinte, $f \in \bigcap_{n \in \mathbb{N}} S_{n}$. Por outro lado, se $f \in \bigcap_{n \in \mathbb{N}} S_{n}$, temos $f \in S_{n}, \forall n \in \mathbb{N}$. Então, dado $n \in \mathbb{N}$, existe $m \geq n$ tal que $f \in S_{m, n}$, isto é,

$$
\sum_{k=0}^{m} \frac{1}{k !}\left\|f^{(k)}\right\|_{\overline{\mathcal{D}}}>n \text {, o que implica que } \sum_{k=0}^{\infty} \frac{1}{k !}\left\|f^{(k)}\right\|_{\overline{\mathcal{D}}}=+\infty .
$$

Logo, $f \in \mathcal{H}$. Em suma, provamos que $\mathcal{H}=\bigcap_{n \in \mathbb{N}} S_{n}$.

Provemos que cada $S_{n}$ é denso em $\mathcal{A}(\mathcal{D})$. De fato, dados $n \in \mathbb{N}, g \in \mathcal{A}(\mathcal{D})$ e $\epsilon>0$, é suficiente provar que $B(g, \epsilon) \cap S_{n} \neq \emptyset$. Com efeito, se $g \in \mathcal{H}, g \in S_{n}$, e portanto $B(g, \epsilon) \cap S_{n} \neq \emptyset$. Por outro lado, se $g \notin \mathcal{H}$, então $\sum_{k=0}^{\infty} \frac{\left\|g^{(k)}\right\|_{\overline{\mathcal{D}}}}{k !}=r<\infty$. Considere $f(z)=\frac{\epsilon}{2} \frac{1}{z-2}$ e $h(z)=\frac{1}{z-2}$. Pelo Lema 
4.2.1, temos $h \in \mathcal{H}$. Além disso, $\left\|f^{(k)}\right\|_{\overline{\mathcal{D}}}=\frac{\epsilon}{2}\left\|h^{(k)}\right\|_{\overline{\mathcal{D}}}$. Então,

$$
f \in \mathcal{H} \text { e }\|f\|_{\overline{\mathcal{D}}}=\frac{\epsilon}{2} \sup _{|z| \leq 1} \frac{1}{|z-2|}=\frac{\epsilon}{2}<\epsilon
$$

Daí, como $f \in \mathcal{H}$, existe $m \in \mathbb{N}$ tal que $\sum_{k=0}^{m} \frac{\left\|f^{(k)}\right\|_{\overline{\mathcal{D}}}}{k !}>r+n$. Portanto,

$$
\sum_{k=0}^{m} \frac{\left\|f^{(k)}+g^{(k)}\right\|_{\overline{\mathcal{D}}}}{k !} \geq \sum_{k=0}^{m} \frac{\left\|f^{(k)}\right\|_{\overline{\mathcal{D}}}}{k !}-\sum_{k=0}^{m} \frac{\left\|g^{(k)}\right\|_{\overline{\mathcal{D}}}}{k !}>r+n-r=n .
$$

Então, $f+g \in S_{n}$. Por conseguinte, $f+g \in B(\epsilon, g) \cap S_{n}$. 


\section{Referências Bibliográficas}

[1] M. Abramowitz, I. Stegun, Handbook of Mathematical Functions with Formulas, Graphs, and Mathematical Tables (U.S. Department of Commerce, Washington, DC, 1964). xi, 1

[2] M. Abtahi, T. G. Honary, On the maximal ideal space of Dales-Davie algebras on infinitely differentiable functions, Bull. London Math. Soc. 39 (2007) 940-948. iii, v, x, xi, 5, 23, 36, 38, $39,40,41,42,43,44$

[3] R. M. Aron, L. Bernal-González, D. M. Pellegrino and J. B. Seoane-Sepúlveda, Lineability. The Search for Linearity in Mathematics, Monographs and Research Notes in Mathematics. Boca Raton, FL, CRC Press, 2016. x, xi, 9, 10, 45, 47

[4] R. M. Aron, V. I. Gurariy, J. B. Seoane-Sepúlveda, Lineability and spaceability of sets of functions on $\mathbb{R}$, Proc. Amer. Math. Soc. 133 (2005) 795-803. x

[5] R. M. Aron, D. Pérez-García, J. B. Seoane-Sepúlveda, Algebrability of the set of non-convergent Fourier series, Studia Math. 175 (2006) 83-90. x

[6] L. Bernal-González, D. Pellegrino and J. B. Seoane-Sepúlveda, Linear subsets of nonlinear sets in topological vector spaces, Bull. AMS. 51 (2014), 71-130. x

[7] W. J. Bland and J. F. Feinstein, Completions of normed algebras of differentiable functions, Studia Math. 170 (2005) 89-111. iii, v, xi, 26, 31

[8] G. Botelho, D. Pellegrino, E. Teixeira, Fundamentos de Análise Funcional, Coleção Textos Universitários, SBM, $2^{\text {a }}$ edição, 2015. xi, 1, 7, 8, 9

[9] M. P. Carmo, Geometria Riemanniana, IMPA. 4

[10] J. B. Conway, Functions of One Complex Variable, Second Edition, Springer, 1978. 12, 57

[11] H. G. Dales, A. M. Davie, Quasianalytic Banach function algebras, J. Funct. Anal 13 (1973) 28-50. iii, v, ix, x, xi, 2, 4, 5, 23, 26, 27, 29, 30, 33, 34, 35

[12] L. Drewnowski, Quasicomplements in F-spaces, Studia Math. 77 (1984) 373-391. 47

[13] R. Engelking, General Topology, Sigma Series in Pure Mathematics, Heldermann Verlag, 1989 xi, $1,2,3$

[14] C. F. Faá di Bruno, Note sur une nouvelle formule de calcul différentiel, Quarterly J. Pure Appl. Math. 1 (1857) 359-360. 1

[15] T. W. Gamelin, Uniform Algebras, Amer. Math. Soc., 2005. xi, 11, 15, 18, 20

[16] V. I. Gurariy, Subspaces and bases in spaces of contiuous functions, Dokl. akad.Nauk SSSR 167(1966),971-973 (Russian) x

[17] V. I. Gurariy and L. Quarta, On lineability os sets of continuous functions, J. Math. Anal. Appl, 294(2004),62-72. x 
[18] T. G. Honary, Relations between Banach function algebras and their uniform closures, Proc. Amer. Soc. 109 (1990), 337-342. iii, v, xi, 19, 35

[19] T. G. Honary and H. Mahyar, Approximation in Lipschitz algebras of infinitely diffentiable functions, Bull. Korean Math. Soc. 36 (1999), 629-636. iii, v, 26

[20] W. P. Johnson, The curious history of Faá di Bruno's formula, Amer. Math. Monthly 109 (2002), 217-234. 1

[21] D.Kitson and R. M. Timoney, Operator ranges and spaceability, J. Math. Anal. Appl. 378 (2011), 680-686. 47

[22] G. M. Leibowitz, Lectures on Complex Function Algebras, Scott, Foresman and Company, Glenviwe, 1970. 11, 13

[23] B. Levine and D. Milman, On linear sets in space $\mathbb{C}$ consisting of bounded variation, Comm. Inst. Sei. Math. Univ. Khark [Zapiski Inst. Mat. Mech.] 16 (1940),102-105 (Russian, with English summary). $\mathrm{x}$

[24] E. L. Lima, Curso de Análise,vol. 1, Projeto Euclides, IMPA, 2012. 1, 42

[25] M.L. Lourenço and D.M.S. Vieira, Algebrability of some subsets of the disk algebra, Bull. Belg. Math. Soc. 23 (2016), 505-514. iii, v, x, xi, 45, 47, 48, 49, 52, 53, 54, 55

[26] M. L. Lourenço and D. M. S. Vieira, Strong algebrabrility and residuality on certain sets of analytic functions, Rocky Mountain Journal of Mathematics (to appear). iii, v, x, xi, 45, 47, $49,50,51,52,55,56,58$

[27] B. D. MacCluer, Elementary Functional Analysis, Springer, 2009. 14, 15

[28] B. Malgrange, Ideals of Differentiable Functions, Oxford University Press, London/New York, 1966. 33

[29] R. E. Megginson, An Introduction to Banach Space Theory, Springer Science \& Business Media, 2012. xi, $6,7,8$

[30] D. M. S. Vieira, Operadores de composição entre as álgebras clássicas de funções analíticas, Dissertação de Mestrado - IME - USP, 2000. 12 